\title{
VIBRATIONAL SPECTROSCOPY OF WATER INTERFACES
}

\author{
QUAN DU \\ Ph.D. Thesis \\ DEPARTMENT OF PHYSICS \\ University of California \\ and \\ MATERIALS SCIENCES DIVISION \\ Lawrence Berkeley Laboratory \\ University of Califormia \\ Berkeley, CA 94720
}

DECEMBER 1994

This work was supported by the Director, Office of Energy Research, Office of Basic Energy Sciences, Materials Sciences Division, of the U.S. Department of Energy under Contract No. DE-AC03-76SF00098. 


\section{DISCLAIMER}

This report was prepared as an account of work sponsored by an agency of the United States Government. Neither the United States Government nor any agency thereof, nor any of their employees, make any warranty, express or implied, or assumes any legal liability or responsibility for the accuracy, completeness, or usefulness of any information, apparatus, product, or process disclosed, or represents that its use would not infringe privately owned rights. Reference herein to any specific commercial product, process, or service by trade name, trademark, manufacturer, or otherwise does not necessarily constitute or imply its endorsement, recommendation, or favoring by the United States Government or any agency thereof. The views and opinions of authors expressed herein do not necessarily state or reflect those of the United States Government or any agency thereof. 


\section{DISCLAIMER}

Portions of this document may be illegible in electronic image products. Images are produced from the best available original document. 


\title{
Abstract \\ Vibrational Spectroscopy of Water Interfaces
}

\author{
by \\ Quan Du \\ Doctor of Philosophy in Physics \\ University of California at Berkeley \\ Professor Yuen Ron Shen, Chair
}

The second order nonlinear optical processes of second harmonic generation and sum frequency generation are powerful and versatile tools for studying all kinds of surfaces. They possess unusual surface sensitivity due to the symmetry properties of the second order nonlinear susceptibility. The technique of infrared-visible sum frequency generation (SFG) is particularly attractive because it offers a viable way to do vibrational spectroscopy on any surfaces accessible to light with submonolayer sensitivity. In this thesis, we applied SFG to study a number of important water interfaces. At the air/water interface, hydrophobic solid/water and liquid/water interfaces, it was found that approximately $25 \%$ of surface water molecules have one of their hydrogen pointing away from the liquid water. The large number of unsatisfied hydrogen bonds contributes significantly to the large interfacial energy of the hydrophobic surfaces. At the hydrophilic fused quartz/water interface and a fatty acid monolayer covered water surface, the structure and orientation of surface water molecules are controlled by the hydrogen bonding of water molecules with the surface $\mathrm{OH}$ groups and the electrostatic interaction with the surface field from the ionization of surface groups. A change of $\mathrm{pH}$ value in the bulk water can significantly change the relative importance of the two interactions and cause a drastic change in orientation of the surface water molecules. SFG has also been applied to study the tribological response of some model lubricant films. Monolayers of Langmuir-Blodgett films were found to disorder orientationaly under mildly high pressure and recover promptly upon removal of the applied pressure.

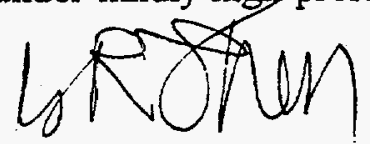




\section{Acknowledgment}

I cannot thank enough my advisor, Professor Y. Ron Shen, for his encouragement, guidance and support. In the past few years, he has been most unselfish with his time and his invaluable advice on science and life. His profound insight and enthusiasm towards physics, clarity of thinking and expression, and optimism towards challenges have set a high standard that I hope to continue following in the future.

I am indebted to Dr. Miquel Salmeron, who in many ways complimented Ron's role as an advisor. His combination of humanity and intellect made him a very pleasant person with whom to work. I will always remember his persistence and optimism when I was almost ready to give up.

I am very thankful to Rich Superfine, who helped me get started with a very demanding laser system. In a way, the short period I worked with him really taught me how things can be done in the lab. Throughout my years in graduate school, I tried very hard to live up to his standard.

I had the remarkable fortune to work with many talented postdocs. Eric Freysz provided much of the support, both emotionally and experimentally, when we were desperately trying to get the laser working and spectra converging. Francis Wolf's expertise on mechanical parts was instrumental in finally getting the Surface Force Apparatus working. Debra Charych was very generous in sharing her invaluable insights on molecules and was always ready to put aside her own work to provide the necessary samples in our attempt to stress monolayers. Xudong Xiao helped me in many ways during my early years in Berkeley. His aggressive determination to get things done proved very effective at a difficult time.

I owe special tributes and thanks to many members of Shen group. Dieter Wilk and Subo Ko spent many hours to make this thesis a more readable one. To them as well 
as Rodney Chin, Xiaowei Zhuang, Chris Mullin, Doseok Kim, Yuanlin Xie, Hiroyuki Saijo, and Lorenzo Marrucci, I am thankful for many pleasant and stimulating discussions on life and science, and for generously sharing equipment which I so desperately needed. Paulo Miranda and George Sefler have made my last year a very productive one, despite much time I spent outside the lab. I only wish that they will be most successful in their own career.

Finally, I would like to thank my parents and my brothers, for their unconditional love and support.

This thesis is dedicated to my girl friend / fiancé / soon-to-be wife, Qing. For the past six and half years, she has been a constant source of love and encouragement for me. Without her, my life will never be as fulfilling and meaningful. 
I. Introduction 1

$\begin{array}{lc}\text { References } & 6\end{array}$

II. Theory and Experimental Implementation of Surface SFG 8

A. Theory 9

A.1 Surface Nonlinear Susceptibility of SFG 9

A.2 Molecular Polarizability 11

A.3 Radiation from the Surface Polarization 15

A.4 Interface and Bulk Contributions 17

B. Experimental Implementation 22

$\begin{array}{ll}\text { References } & 25\end{array}$

$\begin{array}{ll}\text { Figures } & 28\end{array}$

III. Molecular Ordering at the Vapor/Water Interface 31

A. Introduction 31

B. Theory 32

$\begin{array}{ll}\text { C. Experimental Results } & 36\end{array}$

D. Peak Assignment of SFG Spectra 39

E. Analysis of the Free OH Peak 42

F. SFG Spectrum of Water Surface Covered by Alcohol Monolayer 46

G. Temperature Dependence of SFG Spectra of Water Surface 46 
H. Conclusions

References

Figures

IV. Interaction of Water Molecules with Silica Surface

A. Introduction

B. Theory and Experiment

60

C. SFG Spectra and Peak Assignment

D. $\mathrm{pH}$ Dependence of the SFG Spectra

E. Polarization Dependence of the SFG Spectra

F. SFG of Water Surface Covered by a Monolayer of Fatty Acid $\left(\mathrm{C}_{25} \mathrm{COOH}\right)$ 70

G. Conclusions

References

Figures

V. Hydrogen Bonding and Hydrophobicity

$\begin{array}{ll}\text { A. Introduction } & 87\end{array}$

$\begin{array}{ll}\text { B. Theory and Experiment } & 88\end{array}$

C. SFG Spectra of Water Molecules at Hydrophobic Interfaces 89

D. Interfacial Water Structure at Hydrophobic Interfaces 92

E. SFG Spectra of Water Molecules at a Partly Wettable Surface 94

$\begin{array}{ll}\text { F. Conclusions } & 96\end{array}$

$\begin{array}{ll}\text { References } & 97\end{array}$

$\begin{array}{lr}\text { Figures } & 99\end{array}$ 
VI. Disordering of Monomolecular Films Under Pressure

A. Introduction

A.1 Elastic Contact Between Surfaces

A.2 Non-linear Optics

105

B. Experimental

108

B.1 Optical Setup

108

B.2 Pressure Cell

109

B.3 Materials

B.4 Film Preparation Methods

112

C. Results

113

C.1 Control Experiments

113

C. 2 8CB Monolayer Under Pressure

115

C.3 Sum-Frequency Generation Experiments

116

D. Discussion

118

D.1 Confinement and Compression of Molecules

D.2 Vanishing of Second Order Monolayer Susceptibility

References

Figures 


\section{Introduction}

Surface science has undergone an explosive growth over the last decades. Many surface analytical instruments and techniques capable of providing molecular scale information have been developed. ${ }^{1,2}$ Until now, most of the information is gained from scattering or emission of one of the massive particles (electrons, atoms, ions, etc.) from surfaces. This is because the large interaction cross-sections of these particles limit their penetration depth so as to make the processes involved surface specific. ${ }^{2}$ This, however, also limits the possible surfaces to be studied to clean solid surfaces or gas/solid interfaces. Interfaces involving liquids (solid/liquid, gas/liquid and liquid/liquid interfaces), although as important, are much less studied by modern surface science.

Liquid interfaces, in particular the water interfaces, play a very important role in many disciplines of science such as chemistry, biology, astrophysics and atmospheric and soil sciences. Application of surface active agents, such as soaps and detergents, is commonplace in our everyday life. Adhesion, friction and corrosion of solids are greatly influenced by the presence of a thin film of liquid water, whether it is intended or not. ${ }^{3}$ Biological $^{4}$ and electrochemical ${ }^{5}$ processes take place predominantly at solid/water interfaces. On an even greater scale, the composition and the ecology of oceans and fresh waters is, to a large extent, controlled by what is happening at the mineral/water interface. ${ }^{6}$ Furthermore, modern theories of origin of life have suggested that water surfaces played a fundamental role in concentrating the earliest organic material and organizing it into vesicles, which are precursors of the simplest cells. 
Studies of liquid interfaces have a history as old as science itself. The basic equation of capillary was given by Young ${ }^{7}$ and Laplace ${ }^{8}$ in 1805 . Theoretical studies on liquid interfaces have resulted in a good understanding of many interfacial effects, including capillary phenomena and phase transitions. ${ }^{9,10}$ A great deal of effort has also been devoted to elucidate the molecular structure at the aqueous interfaces. Electrostatic interaction was assumed to be responsible for the molecular orientation and surface potential at the vapor/liquid interface. ${ }^{11}$ In the past, all the interfacial properties of the metal electrode/water interface were attributed to a monolayer of solvent molecules immediately adjacent to the metal surface, and a very sophisticated model was developed based on the differential capacitance measurements. ${ }^{5}$ Only recently the effects due to the response of the metal surface to the high external field have been recognized. ${ }^{12}$ The application of powerful computer simulation techniques to liquid interfaces is especially impressive. Molecularly detailed information is now available on many different systems with different interaction potential models and different simulation sizes. ${ }^{13}$ It is essential that they can be compared with experimental investigations with molecular scale information so that improvement can be made in the right direction.

Development of new experimental techniques capable of providing molecular scale information has been very slow. This is largely due to the many conflicting requirements one has to meet in order to study the liquid interfaces. To discriminate against the large background of the bulk molecules, one usually must use particles with large interaction cross-sections as in surface science. Unfortunately this is hampered by the presence of another condensed medium or the dense vapor unavoidably present for any liquid. In the past, researchers relied on optical probes that have a long penetration depth, and used 
sophisticated difference or modulation techniques to extract the interface signal. There has been limited success with this kind of approach in infrared spectroscopic studies of aqueous electrode interface. ${ }^{14}$ The best results have been obtained with adsorbed species at these interfaces. However, even in these cases, the interpretation has been difficult. Surface Enhanced Raman Spectroscopy was thought to be ideal because the great enhancement from the surface species automatically provides the necessary surface specificity. However, only a limited number of systems can be studied by this technique, and the surface usually has to go through some treatment whose exact effect is hard to characterize.$^{15}$ Reflection and scattering of $\mathrm{X}$-rays near grazing incidence has been widely used to characterize liquid surfaces and interfaces. Most of these experiments were performed with synchrotron radiation because of its high brightness. ${ }^{16,17}$ The density distribution along the normal direction, roughness of the liquid surface, and the long range structural ordering along the surface plane can be studied in great detail. ${ }^{17}$ Experiments using a surface force apparatus which measures the small force between two atomicaly flat surfaces immersed in a liquid, have also provided a great deal of insights to the structure and layering of liquid molecules adjacent to a solid wall. ${ }^{18}$ Scanning probe microscopes (STM and AFM) are also excellent tools for studying solid/liquid interfaces, ${ }^{19}$ due to the relative ease of the measurement and the direct information available. However it is very difficult for the STM and AFM to directly detect the mobile liquid molecules.

Recently, optical second harmonic generation (SHG) and sum-frequency generation (SFG) have been proven to be extremely versatile surface probes, with excellent surface sensitivity and specificity. ${ }^{20}$ These techniques are based on the fact that within the dipole approximation the second order nonlinear optical process is not allowed in a medium with 
inversion symmetry, but at the surfaces and interfaces, the inversion symmetry is always broken so that the second order nonlinear optical processes are allowed. As surface probes, both SHG and SFG are sufficiently sensitive to detect a submonolayer of atoms or molecules. In addition, as coherent nonlinear optical processes, SHG and SFG posses all the advantages of laser spectroscopic techniques, including excellent spectral and temporal resolution. For study of liquid interfaces involving water or organic molecules, SFG is a better choice because of the molecular selectivity it provides. ${ }^{21}$ In this process, a visible laser with frequency $\omega_{1}$ is mixed with a tunable infrared laser with frequency $\omega_{2}$ to generate the output with frequency at $\omega_{3}=\omega_{1}+\omega_{2}$. The vibrational spectrum of the interface can be obtained by tuning the infrared laser through molecular resonances of the interfacial molecules. Because of the intrinsic ability of SFG to discriminate against the bulk contribution, the measurement of the surface spectrum is possible even for the case of pure liquids.

This thesis covers some of our efforts to study liquid interfaces, especially water interfaces, using SFG as a vibrational spectroscopic technique. In chapter two we will discuss the theory of SFG starting from second order perturbation theory, and how multipole contributions of bulk origin cannot be ignored from theoretical point of view. At the end of chapter 2, we will also discuss the experimental implementation of SFG for surface studies. Molecular orientation and interaction of water molecules at a number of important interfaces are described in chapters 3-5. In chapter 3, our SFG study reveals that, at the vapor/water interface, about $25 \%$ of surface water molecules have one of their hydrogens pointing towards the vapor side. The interfacial molecular structure of water at this interface is found to be very similar to that of oil/water and water/hydrophobic solid 
interfaces (chapter 5), which are characterized by the hydrophobicity of the interface. In chapter 4 , we study the water molecules at the hydrophilic silica/water and fatty acid monolayer/water interfaces. At these interfaces, behavior of water molecules is influenced strongly by the presence of the surface electric field resulting from the ionization of some surface groups. These studies represent the most detailed information available experimentally for these interfaces, and can be directly compared with computer simulations. Along a very different direction, SFG has been applied to study the tribological response of some model lubricant films in chapter 6 . Monolayers of LangmuirBlodget films and self-assembled films are found to become disordered orientationaly under mildly high pressure. Upon removal of the pressure, however, the molecules are able to recover their orientation completely. 


\section{Reference}

1. G. A. Somorjai, Chemistry in Two Dimensions: Surfaces, (Cornell University Press, Ithaca, New York, 1981).

2. D. P. Woodruff and T. A. Delchar, Modern Techniques of Surface Science, (Cambridge University Press, New York, 1986).

3. E. Mattsson, Basic Corrosion Technology for Scientists and Engineers, (Ellis Horwood Limited, New York, 1989).

4. R. Pethig, in Annual Review of Physical Chemistry, Vol. 43 ( 1992 ) pp. 177, edited by H. L. Strauss.

5. W. R. Fawcett, J. Chem. Phys. 93 (9), 6813 ( 1990 ); J. O M. Brockris and S. U. M. Khan, Quantum Electrochemistry ( Plenum, New York, 1979 ), Chapter 1; N. F. Mott and R. J. Watts - Tobin, Electrochemica Acta, 4, 79 ( 1961 ).

6. W. Stumm, Chemistry of the Solid-Water Interface, (John Wiley \& Sons, Inc., New york, 1992).

7. T. Young, Miscellaneous Works, G. Peacock, ed., J. Murray, London, 1855, Vol. I, p.418.

8. P.S. de Laplace, Mechanique Celeste, Suplement to Book 10, 1806.

9. J. S. Rowlison and B. Widom, Molecular Theory of Capilarity, (Claredon, Oxford, 1982).

10. D. Beaglehole, in Fluid Interfacial Phenomena, C. A. Croxton Ed., (Wiley, New York, 1986), p.523. 
11. F. H. Stillinger, Jr. And A. Ben Naim, J. Chem. Phys. 47, 4431 (1967).

12. W. Schmickler and D. Henderson, Prog. Surf. Sci. 22, 232 (1986); P. Gies and R. R. Gerhardts, Phys. Rev. B 33, 982 (1986).

13. For a recent review, see A. Pohorille and M. A. Wilson, J. Molecular Structure 284, 271 (1993).

14. A. Bewick, K. Kunimatsu, B. S. Pons and J. W. Russeil, J. Electroanal. Chem., 160, 47 (1984); A. Bewick, in Trends in Interfacial Electrochemistry, A. F. Silva eds., (D. Reidel Publishing Company, Boston, 1986).

15. R. K. Chang, and T. E. Furtak, eds. Surface Enhanced Raman Scattering, (Plenum, New York, 1982); M. Moskovits, Rev. Mod. Phys. 57, 783 (1985).

16. P. N. Ross, in Structure of Electrified Interfaces, J. Lipkowski, P. N. Ross eds., chapter 3 (VCH Publishers, INC., New York, 1993).

17. P.S. Pershan, Physica A 200, 50 (1993).

18. For a recent review see, J. N. Israelachvili, Surf. Sci. Rep. 14, 109 (1992).

19. O. M. Magnussen, J. Hotlos, B. J. Behm, N. Batina and D. M. Kolb, Surf. Sci. 296, 310 (1993).

20. Y.R. Shen, Annu. Rev. Mat. Sci. 16, 69 (1986); Y.R. Shen, Nature, 337, 519 (1989); Y.R. Shen, Annu. Rev. Phys. Chem. 40, 327 (1989).

21. R. Superfine, J. Y. Huang and Y. R. Shen, Phys. Rev. Lett. 66, 1066 (1991). 


\section{Theory and Experimental Implementation of Surface SFG}

Surface SFG was first developed as a natural extension of the surface optical second harmonic generation. Although it has excellent surface specificity and submonolayer sensitivity, SHG using visible or near infrared lasers does not have enough molecular specificity, because electronic transitions are in general too broad to be used to identify molecules. SHG using infrared lasers to study vibrational modes of molecules, on the other hand, is very difficult because the output is in the infrared and detectors with sufficient sensitivity are not readily available. The problem is easily solved in sum frequency generation by using a visible laser and a tunable infrared laser. In this case, molecules can be selectively probed by vibrational spectroscopy, while submonolayer sensitivity can be achieved because very low intensities of visible SF signal can be detected using a photomultiplier tube. The first experimental demonstration of SFG was performed by X. D. Zhu et al in $1987 .{ }^{1}$ Since then SFG has been successfully applied to studies of surface vibrational structures of many different systems and dynamics at various interfaces. ${ }^{2}$ In this chapter we will discuss the basic theory behind the successful application of SFG as a versatile surface probe and experimental setup as they are relevant to this thesis. 


\section{A. Theory}

\section{A.1 Surface Nonlinear Susceptibility of SFG}

Although sum-frequency generation in general refers to any three wave mixing process where the two input lasers have different frequencies, we limit our discussion to the case where at least one of the input lasers is close to a resonance of molecular vibrational mode. In the presence of two optical electric fields, $\mathbf{E}\left(\omega_{1}, t\right)=\mathbf{E}\left(\omega_{1}\right)$ $\operatorname{Exp}\left(-i \omega_{1} t\right)+E^{*}\left(\omega_{1}\right) \operatorname{Exp}\left(i \omega_{1} t\right)$ and $E\left(\omega_{2}, t\right)=E\left(\omega_{2}\right) \operatorname{Exp}\left(-i \omega_{2} t\right)+E^{*}\left(\omega_{2}\right) \operatorname{Exp}\left(i \omega_{2} t\right)$, an electric dipole polarization

$$
P\left(\omega_{\mathbf{s}}\right)=\chi_{\mathbf{D}}^{(2)}: \mathbf{E}\left(\omega_{1}\right) \mathbf{E}\left(\omega_{2}\right)
$$

is set up in the medium and oscillates at the frequency $\omega_{\mathrm{s}}=\omega_{1}+\omega_{2}{ }^{3-5}$ Here $\chi_{\mathrm{D}}^{(2)}$ is the second order susceptibility of the medium. For now we limit all our discussion to within the dipole approximation, so that $\chi_{\mathbf{D}}^{(2)}$ is completely a material property. For a bulk medium with inversion symmetry, $\chi_{\mathbf{D}}^{(2)}$ must be an invariant under inversion. With $\mathbf{E}\left(\omega_{1}\right)$, $\mathbf{E}\left(\omega_{2}\right)$ and $\mathbf{P}\left(\omega_{s}\right)$ change signs under inversion, the only possible way Eq.(2.1) can be true is that $\chi_{D}^{(2)}$ is zero. This same argument can also be applied to systems with limited symmetry to determine the nonvanishing elements of $\chi_{D}^{(2)}$. At interfaces, where the inversion symmetry is necessarily broken, $\chi_{\mathbf{D}}^{(2)}$ will not vanish. This is the fundamental reason why SFG is a surface specific process. However it is also important to remember that for multipole contributions, it is possible to have nonvanishing $\chi^{(2)}$ for any medium, as will be discussed later. 
For the interfacial molecules or the molecular adsorbates on the surface which are situated in an asymmetric environment, $\chi_{\mathbf{D}}^{(2)}$ can be related to the molecular polarizability $\alpha_{\operatorname{lmn}}^{(2)} \cdot 3$

$\chi_{\mathrm{jik}}^{(2)}=\mathrm{N} \cdot \sum_{\mathrm{l}, \mathrm{m}, \mathrm{n}}<(\hat{\mathrm{i}} \cdot \hat{\mathrm{l}})(\hat{\mathrm{j}} \cdot \hat{\mathrm{m}})(\hat{\mathrm{k}} \cdot \hat{\mathrm{n}})>\alpha_{\mathrm{lmn}}^{(2)}$

here $\mathrm{N}$ is the density of the molecules, and the angular braces indicate the coordinate transformation from the molecularly fixed axis to the lab frame, averaged over the molecular orientational distribution. For the molecules having nonvanishing $\alpha_{\operatorname{lmn}}^{(2)}$ (this in turn can be related to symmetry characteristics of the molecules, as will be shown below), $\alpha_{\operatorname{lmn}}^{(2)}$ vanishes in the bulk as the angular average does for a homogeneous medium. Except for the case of molecular adsorbates on surfaces, the orientational average is usually expected to be non zero for several molecular layers close to a surface. Because the width of the interface is much smaller than the wavelength of the radiation, it is convenient to define an effective surface susceptibility $\chi_{s}^{(2)}$ to describe the total response of the interface integrated over the transition layers, ${ }^{6}$

$\chi_{s}^{(2)}=\int_{s} \chi^{(2)} d z=<n>\cdot N_{s} \cdot \sum_{l, m, n}<(\hat{i} \cdot \hat{l})(\hat{j} \cdot \hat{m})(\hat{k} \cdot \hat{n})>\alpha_{l m n}^{(2)}$

where $\mathrm{N}_{\mathrm{s}}$ is the surface number density of molecules. Without a priori information about the orientational decay from the interface into the bulk, it is hard to determine whether to 
associate the $\chi_{s}^{(2)}$ to many layers of molecules with small preferred average orientation or to a monolayer of molecules with a much better defined orientation. Up to now, it has always been assumed that the molecules adopt an average orientation, and the decay into the bulk is taken into account by $<\mathrm{n}>$, the average number of layers of molecules having preferred orientation. For most of the cases, $<\mathrm{n}>$ is found to be around 1 . However, this by no means is suggesting that SFG is only sensitive to the outermost layer of molecules.

\section{A.2 Molecular Polarizability}

The microscopic expression for the molecular polarizability can be obtained from the standard second order perturbation theory. ${ }^{7,8}$ For the case of only one frequency, $\omega_{2}$, on resonance, $\alpha_{\mathrm{lmn}}^{(2)}$ can be written as $\alpha_{\mathrm{lmn}}^{(2)}=\alpha_{\mathrm{lmn}}^{(2) \mathrm{NR}}+\alpha_{\mathrm{lmn}}^{(2) \mathrm{R}}$, where $\alpha_{\mathrm{lmn}}^{(2) \mathrm{NR}}$ is the non-resonant part and $\alpha_{\operatorname{lmn}}^{(2) R}$ the resonant part. The resonant contribution can be expressed: ${ }^{9}$

$\alpha_{\mathrm{lnn}}^{(2) R}\left(\omega_{\mathrm{s}}=\omega_{1}+\omega_{2}\right)=\frac{\mathrm{i}}{\hbar} \sum_{\mathrm{v}} \sum_{\mathrm{v}^{\prime}} \frac{(\hat{\rho})_{\mathrm{gv}, \mathrm{gv}}\left\langle\psi_{\mathrm{gv}}\left|\mu_{\mathrm{n}}\right| \psi_{\mathrm{gv}}\right\rangle\left\langle\psi_{\mathrm{gv}}\left|\alpha_{\mathrm{lm}}^{(1)}\right| \psi_{\mathrm{gv} v^{\prime}}\right\rangle}{\mathrm{i}\left(\omega_{\mathrm{gv}^{\prime}, \mathrm{gv}}-\omega_{2}\right)+\Gamma_{\mathrm{gv} \mathrm{g}^{\prime} \mathrm{gv}}}$

where $\hat{\rho}$ is the density matrix, $\mu_{n}$ is the nth component of the electric dipole moment operator, $\alpha_{\mathrm{lm}}^{(1)}$ is the polarizability tensor, and $\omega_{\mathrm{gv}, \mathrm{gv}}\left(\mathbf{h} \omega_{\mathrm{gv}^{\prime}, \mathrm{gv}}=\mathbf{E}_{\mathrm{gv} v^{\prime}}-\mathbf{E}_{\mathrm{gv}}\right)$ and $\Gamma_{\mathrm{gv}^{\prime}, \mathrm{gv}^{\prime}}$ are the resonance frequency and phenomenological damping constant of the vibrational transitions, respectively. Vibrational modes show up as resonances with Lorenzian line shape in the sum frequency generation spectrum when $\omega_{2}$ is tuned through resonances. It 
should be noted that because the SFG signal is proportional to the square of $\alpha_{\mathrm{lmn}}^{(2)}$, the presence of the non resonant term in $\alpha_{\operatorname{lmn}}^{(2)}$ can significantly distort the SFG spectrum so a proxy to the $\mathbb{R}$ or Raman spectrum is not guaranteed. Within the Born-Oppenheimer approximation, $\left|\Psi_{\mathrm{gv}}\right\rangle=\left|\varphi_{\mathrm{g}}\right\rangle\left|\Theta_{\mathrm{gv}}\right\rangle$, where $\left|\varphi_{\mathrm{g}}\right\rangle$ and $\left|\Theta_{\mathrm{v}}\right\rangle$ represent the electronic and rovibrational states, respectively. Eq.(2-4) can be then simplified to:

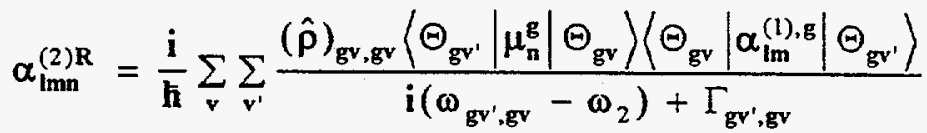

here $\mu_{\mathrm{n}}^{\mathrm{g}}$ and $\alpha_{\mathrm{lm}}^{(1), \mathrm{g}}$ represent the dipole moment and polarizability of the ground electronic state. Within the Condon approximation, $\boldsymbol{\alpha}_{\mathbf{l m}}^{(1), g}$ can be expressed as:

$\alpha_{1 \mathrm{~m}}^{(\mathrm{l}), \mathrm{g}}=\frac{1}{\hbar} \sum_{\mathbf{a}}\left[\frac{\left\langle\mathrm{g}\left|\mu_{1}\right| \mathbf{a}\right\rangle\left\langle\mathbf{a}\left|\mu_{\mathrm{m}}\right| \mathbf{g}\right\rangle}{\omega_{\mathrm{ag}}-\omega_{\mathrm{s}}}+\frac{\left\langle\mathrm{g}\left|\mu_{\mathrm{m}}\right| \mathbf{a}\right\rangle\left\langle\mathbf{a}\left|\mu_{1}\right| \mathbf{g}\right\rangle}{\omega_{\mathrm{ag}}+\omega_{\mathrm{s}}}\right]$

here $\mathrm{a}, \mathrm{g}$ denote the electronic states only. To bring our expression to the IR or Raman language, it is helpful to simplify the expression of the resonant contribution by using the Plazcek's approximation, in which $\mu_{\mathrm{n}}^{\mathrm{g}}$ and $\boldsymbol{\alpha}_{\mathrm{lm}}^{(1), \mathrm{g}}$ are expanded in terms of normal coordinates $\mathbf{Q}_{1}$. Then,

$\mu_{\mathrm{n}}^{\mathrm{g}}=\mu_{\mathrm{n} 0}^{\mathrm{g}}+\sum_{1}\left(\frac{\partial \mu_{\mathrm{n}}^{\mathrm{g}}}{\partial \mathbf{Q}_{1}}\right)_{0} \mathbf{Q}_{1}+\cdots$

and 


$$
\alpha_{1 \mathrm{~lm}}^{(1), \mathbf{g}}=\alpha_{1 \mathrm{~m} 0}^{(1), g}+\sum_{1}\left(\frac{\partial \alpha_{1 \mathrm{~m}}^{(1), g}}{\partial \mathbf{Q}_{1}}\right)_{0} \mathbf{Q}_{1}+\cdots
$$

With this approximation, eq. (2-5) becomes:

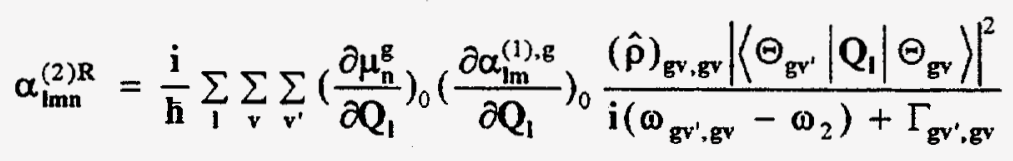

If we are only concerned with the transitions from the vibrational ground state to the first excited vibrational level, the expression can be further simplified to:

$$
\alpha_{\operatorname{lmn}}^{(2) R}=\sum_{q} \frac{A_{q}}{\left(\omega_{q}-\omega_{2}-i \Gamma_{q}\right)} \Delta \rho_{g q}
$$

where

$$
\mathbf{A}_{\mathrm{q}}=\frac{1}{2 \omega_{\mathrm{q}}} \frac{\partial \mu_{\mathrm{n}}}{\partial \mathbf{Q}} \frac{\partial \alpha_{\mathrm{m}}^{(1)}}{\partial \mathbf{Q}}
$$

and $\Delta \rho_{\mathrm{gq}}$ is the population difference between the vibrational ground and excited states, $\frac{\partial \mu_{\mathrm{n}}}{\partial \mathbf{Q}}$ and $\frac{\partial \alpha_{\mathrm{lm}}^{(1)}}{\partial \mathbf{Q}}$ are the infrared dipole moment derivative and Raman polarizability for the normal mode Q. Eq. (2.6) explicitly provides us with the selection rule for sum frequency generation spectroscopy. The normal mode must be both infrared and Raman active in order to be detected by SFG. This is consistent with the requirement from symmetry considerations that there must be a lack of centrosymmetry in the molecule itself in order for it to have non zero $\alpha_{\operatorname{lmn}}^{(2)}$. It is well known that for a molecule having inversion 
symmetry, the infrared active and Raman active modes are mutually exclusive. Only a molecule without centrosymmetry can have normal modes that are both Raman and infrared active. $^{10}$

It is also useful to relate $\alpha^{(2) R}$ to the differential Raman scattering cross section $\frac{d \sigma}{d \Omega}$. On resonance we have ${ }^{11}$

$$
\alpha^{(2) R}=\frac{c^{2}}{\hbar \sqrt{\omega_{s} \omega_{1}^{3}}} \frac{\mu_{q}}{\Gamma_{q}}\left(\frac{d \sigma}{d \Omega}\right)^{\frac{1}{2}}
$$

where $\mu_{q}$ is the infrared transition dipole moment. We can use this expression to estimate $\alpha^{(2) \mathrm{R}}$ from the symmetric vibration of the $\mathrm{CH}_{3}$ group and $\chi_{s}^{(2)}$ of a monolayer of model molecules, e.g. a monolayer of fatty acid molecules on the water surface. From the literature we have $\mu_{q} \sim 0.05 \mathrm{D}\left(1\right.$ Debye $\left.=10^{-18} \mathrm{esu}\right),{ }^{12}$ the differential Raman cross section $\frac{\mathrm{d} \sigma}{\mathrm{d} \Omega} \sim 5.3 \times 10^{-30} \mathrm{~cm}^{2} \mathrm{sr}^{-1},{ }^{13}$ and for the resonance width and peak position, $\Gamma_{\mathrm{q}} \sim 10 \mathrm{~cm}^{-1}$ and $\omega_{\mathrm{q}} \sim 2875 \mathrm{~cm}^{-1}$, respectively. For our laser system, $\omega_{1} \sim 3.5 \times 10^{15} \mathrm{~s}^{-1}\left(\lambda_{1}=0.532 \mu \mathrm{m}\right)$, therefore $\omega_{\mathrm{s}}=4.04 \times 10^{15} \mathrm{~s}^{-1}$ on resonance. Inserting these values into Eq. (2.7) we obtain $\alpha^{(2) R} \sim 3.6 \times 10^{-30}$ esu. With the surface number density $\mathrm{N}_{\mathrm{s}} \sim 5 \times 10^{14} \mathrm{~cm}^{-2}$ for a Langmuir film of fatty acid, we can obtain $\chi_{s}^{(2)}=1.8 \times 10^{-15}$ esu, which should be easily detectable as susceptibility of $\sim 2 \times 10^{-17}$ esu is measurable in SHG.

So far we have discussed the situation where only one of the two input laser frequencies, $\omega_{2}$, is on resonance. When a tunable visible or UV laser is used, $\omega_{1}$ can be on 
resonance with one of the electronic excitations. In this case $\chi_{\mathrm{s}}^{(2)}$ can be further enhanced by another factor of $\sim 10-100$. It is relatively easy to derive the expression of $\alpha^{(2) R}$ for doubly resonant SFG:

$$
\alpha_{\mathrm{lmn}}^{(2) \mathrm{R}}=\frac{1}{\hbar^{2}}\left[\left\langle\mathrm{k}\left|\mu_{1}\right| \mathrm{g}\right\rangle\left\langle\mathrm{g}\left|\mu_{\mathrm{m}}\right| \mathrm{k}\right\rangle\right] \sum_{\mathrm{q}} \frac{\left(\frac{\partial \mu_{\mathrm{n}}^{\mathrm{g}}}{\partial \mathrm{Q}}\right)_{0} \sqrt{\frac{\hbar}{2 \omega_{\mathrm{q}}}}}{\left(\omega_{\mathrm{q}}-\omega_{2}-\mathrm{i} \Gamma_{\mathrm{q}}\right)} \sum_{\mathrm{v}} \sum_{\mathrm{u}} \frac{(\hat{\rho})_{\mathrm{gv}, \mathrm{gv}}\left\langle\Theta_{\mathrm{gv}} \| \Theta_{\mathrm{ku}}\right\rangle\left\langle\Theta_{\mathrm{ku}} \| \Theta_{\mathrm{gv}}\right\rangle}{\left(\omega_{\mathrm{ku}, \mathrm{gv}}-\omega_{2}-\omega_{1}-\mathrm{i} \Gamma_{\mathrm{kg}}\right)}
$$

Here for simplicity we have restricted ourselves to the case in which one of the vibrational levels $\left(v, v^{\prime}\right)$ is the ground state. It should be noted that the line shape of resonant SFG is much more complicated. However, if both $\omega_{2}$ and $\omega_{1}$ are tuned such that $\omega_{\mathrm{s}}=\omega_{1}+\omega_{2}$ is a constant, the usual Lorenzian line shape can be recovered. On the other hand, if $\omega_{2}$ is fixed and $\omega_{1}$ varied, the SFG spectrum will be closely related to the one-photon spectrum of the $g \rightarrow k$ transition.

\section{A.3 Radiation from the Surface Polarization}

The polarization produced by the effective surface susceptibility $\chi_{3}^{(2)}$ can be idealized as a sheet with dielectric constant $\varepsilon^{\prime}(\omega)$ at the interface between media 1 and 2 . Assuming the two input lasers are incident from medium 1 into medium 2 , the optical field radiated from the surface into medium 1 can then be written as: ${ }^{3}$

$$
\mathbf{E}(r, t)=i 2 \pi \frac{\omega_{s}}{c} \frac{\sec \theta_{s}}{\sqrt{\varepsilon_{1}^{s}}}\left(L_{s} \cdot e_{s} \chi_{s}^{(2)}: L_{1} \cdot e_{1} L_{2} \cdot e_{2}\right) E_{1} E_{2} \exp \left(i k_{1 s} \cdot r-i \omega_{s} t\right)
$$


where $\varepsilon_{1}^{s}$ is the dielectric constant of medium $I$ at $\omega_{s}, \mathbf{e}_{\mathrm{i}}$ and $E_{\mathrm{i}}$ are the unit vector and amplitude of the optical field, respectively, for the field at $\omega_{\mathrm{i}}$, and $\theta_{\mathrm{s}}$ and $\mathbf{k}_{\mathrm{ls}}$ are the angle with respect to the surface normal and the wavevector for the reflected sum frequency beam in medium 1, respectively. Because sum frequency generation is a coherent three wave mixing process, the output is well collimated and the wavevector $\mathbf{k}_{1 \mathrm{~s}}$ is determined by the momentum conservation condition, $\mathrm{k}_{\mathrm{s}, \mathrm{x}}=\mathrm{k}_{1, \mathrm{x}}+\mathrm{k}_{2, \mathrm{x}}$, where $\mathrm{k}_{\mathrm{i}, \mathrm{x}}$ is the component of the wavevector of the field at $\omega_{i}$ parallel to the interface. The macroscopic local field factors, $\mathbf{L}_{\mathrm{i}}$, relate the incident field at $\omega_{\mathrm{i}}$ in medium 1 to the field inside the polarization sheet. They are given $b y^{3}$

$L_{x x}=\frac{2 \varepsilon_{1} k_{2 z}}{\varepsilon_{2} k_{1 z}+\varepsilon_{1} k_{2 z}}$

$L_{z z}=\frac{2 \varepsilon_{1} k_{1 z}}{\varepsilon_{2} k_{1 z}+\varepsilon_{1} k_{2 z}}\left(\frac{\varepsilon_{2}}{\varepsilon^{\prime}}\right)$

$\mathrm{L}_{\mathrm{yy}}=\frac{2 \mathrm{k}_{1 z}}{\mathrm{k}_{\mathrm{iz}}+\mathrm{k}_{2 \mathrm{z}}}$,

where the dielectric constants and wavevectors are evaluated at the respective frequencies. Although not widely recognized, it is possible that considerable distortion of the SFG spectrum can be introduced by the macroscopic local field factors, especially when SFG is applied to study an interface where the bulk has very strong resonances close in 
frequency to those of the surface molecules, as in the case of pure liquid interfaces. One example which will be discussed is the air/water interface (see chapter 3 ).

Eq.(2.9) allows us to calculate the signal of SFG in photons per pulse. Assuming both input beams have the same pulse duration $\mathrm{T}$ and area $\mathrm{A}$, we have

$$
S\left(\omega_{s}\right)=8 \pi^{3} \frac{\omega_{s} \sec ^{2} \theta_{s}}{\hbar c^{3}\left(\varepsilon_{1}^{s} \varepsilon_{1}^{1} \varepsilon_{1}^{2}\right)^{1 / 2}}\left|\mathbf{e}_{s}^{\prime} \cdot \chi_{s}^{(2)}: \mathbf{e}_{1}^{\prime} \mathbf{e}_{2}^{\prime}\right|^{2} \frac{U_{1} U_{2}}{A T}
$$

where $\mathbf{e}_{1}^{\prime}=\mathbf{L}_{\mathrm{i}} \cdot \mathbf{e}_{\mathrm{i}}$. As an example of the signal for a typical experiment, we use the parameters of our laser system. Typical energies of the laser pulses at the surface are 0.5 $\mathrm{mJ}$ at $0.532 \mu \mathrm{m}$ and $100 \mu \mathrm{J}$ in the infrared. The pulses are about 20 ps in duration and are focused to a beam size of $400 \mu \mathrm{m}$ in diameter at the surface. For a dielectric surface, with the laser beams incident at $\sim 45^{\circ}$, the local field factors are of order unity. With $\chi_{3}^{(2)} \sim 10^{-15}$ esu (estimated above for a compact monolayer of $\mathrm{CH}_{3}$ groups), a signal of $\sim 500$ photons per pulse is expected. With detection efficiency of $\sim 10 \%$ and typical scattering background noise of $\sim 0.01$ photon/pulse, signal to noise ratio of $\sim 10^{4}$ can be achieved. This implies that one can detect even $1 \%$ of a monolayer. The experimental setup will be discussed in the following section.

\section{A.4 Interface and Bulk Contributions}

So far we have only considered contributions to SFG from the interfacial molecules. This is justified within the dipole approximation, as has been shown above for centrosymmetric media. However, because the density and structure across the interface 
vary significantly over several atomic layers, the electric field and nonlinear susceptibilities from higher order multipole contributions have very large gradients along the surface normal. Therefore, the contributions from higher multipoles can be the same order of magnitude as the dipole contributions from the interfacial molecules. ${ }^{6,14,15}$

The theory of multipolar contributions to SHG or SFG in reflection has been studied extensively. The effective nonlinear polarization can be expanded into a series of multipole terms, ${ }^{6,14,15}$

$\mathbf{P}_{\text {eff }}^{(2)}\left(\omega_{\mathrm{s}}=\omega_{1}+\omega_{2}\right)=\mathbf{P}^{(2)}\left(\omega_{\mathrm{s}}\right)-\nabla \cdot \overline{\mathbf{Q}}^{(2)}\left(\omega_{\mathrm{s}}\right)+\frac{\mathrm{c}}{\mathrm{i} \omega_{\mathrm{s}}} \nabla \times \mathbf{M}^{(2)}\left(\omega_{\mathrm{s}}\right)+\cdots$

where $\mathbf{P}, \overrightarrow{\mathbf{Q}}$, and $\mathbf{M}$ denote the polarization due to electric-dipole, electric-quadrupole, and magnetic dipole contributions, respectively. The expansion is valid in the bulk when the volume elements used to define the average macroscopic polarization are smaller than the typical length of field variation. It should be clear that at the interface, terminating the expansion at a certain order is not justified. We will discuss this point further, but it will be helpful for now to restrict our discussion to the expansion up to the first derivative. $\mathbf{P}_{\text {eff }}^{(2)}$ can be written as: ${ }^{14}$

$$
\begin{aligned}
& \mathbf{P}^{(2)}\left(\omega_{s}\right)=\chi^{\mathrm{D}}: \mathbf{E}\left(\omega_{1}\right) \mathbf{E}\left(\omega_{2}\right)+\chi^{\mathrm{P}_{1}}: \nabla \mathbf{E}\left(\omega_{1}\right) \mathbf{E}\left(\omega_{2}\right)+\chi^{\mathrm{P}_{2}}: \mathbf{E}\left(\omega_{1}\right) \nabla \mathbf{E}\left(\omega_{2}\right) \\
& \mathbf{Q}\left(\omega_{s}\right)=\chi^{\mathrm{Q}}: \mathbf{E}\left(\omega_{1}\right) \mathbf{E}\left(\omega_{2}\right) \\
& \mathbf{M}\left(\omega_{s}\right)=\chi^{\mathrm{M}}: \mathbf{E}\left(\omega_{1}\right) \mathbf{E}\left(\omega_{2}\right)
\end{aligned}
$$


where the term $\chi^{D}$ is the contribution due to interfacial molecules as discussed earlier. The microscopic expressions for multipolar susceptibilities can be found in literature, ${ }^{8}$ and they are obtained by replacing one dipolar operator with an electric quadrupole or magnetic dipole operator. It is easy to see that the terms $\chi^{\mathrm{P}}, \chi^{\mathrm{Q}}$, and $\chi^{\mathrm{M}}$ are not forbidden by any crystal symmetry. These are the terms responsible for bulk related contributions to SFG. As an example, we examine the contribution of $\chi^{\mathrm{P}}$ term to SFG when the polarizations are $\mathrm{s}, \mathrm{s}$, and $\mathrm{p}$ for the sum frequency output, visible input, and infrared input, respectively. The effective susceptibility through bulk polarization can be written as, ${ }^{15}$

$\chi_{y y z}^{b}=\frac{k_{1}}{k_{s z}^{2}+k_{1 z}+k_{2 z}} \frac{\chi_{y y z z}^{\mathrm{p}_{2}}}{\varepsilon_{2}\left(\omega_{2}\right) \sin \theta_{2}} \sin \left(\theta_{2}-\theta_{1}\right)$

where $\theta_{2}$ and $\theta_{1}$ are the incident angles of the infrared and visible lasers. If the separation between them are made sufficiently small the contribution from the bulk polarization can be safely neglected. What is more difficult to separate from the surface dipole term is the quadropole contribution due to the great field gradient along the surface normal. In this case, the effective surface polarization is ${ }^{6,14}$

$$
P_{\text {eff }, y}^{s}=\int_{I} \chi_{y y z z}^{p_{2}} E_{y}\left(\omega_{1}\right) \frac{d E_{z}\left(\omega_{2}\right)}{d z} d z=E_{y}\left(\omega_{1}\right) D_{z}\left(\omega_{2}\right) \int \chi_{I} x_{y y z z}^{P_{2}} \frac{d}{d z}\left(\frac{1}{\varepsilon}\right) d z
$$


where we notice that $\mathrm{y}$ component of field and $\mathrm{z}$ component of displacement field are continuous across the interface. It is difficult to find an exact analytical result for the integral because both $\chi_{y y z z}^{\mathrm{P}_{2}}$ and $1 / \varepsilon$ are complex functions of $z$ across the interface. However, for air/liquid interfaces, it is reasonable to assume that both $\chi_{\text {yyzz }}^{\mathrm{P}_{2}}$ and $\varepsilon$ are linear functions of density. We have

$\chi_{y y z z}^{\mathrm{P}_{2}}=\chi_{y y z z 0}^{\mathrm{P}_{2}} \frac{\rho}{\rho_{0}}$

$\varepsilon=1+\left(\varepsilon_{0}-1\right) \frac{\rho}{\rho_{0}}$

here only $\rho$ is a function of $z$, while the material constants assume their respective values in the bulk. After substituting Eq.(2.16) into Eq.(2.15) and carrying out a simple integration, we have,

$P_{\text {eff } y}^{\mathrm{s}}=\mathrm{E}_{\mathrm{y}}\left(\omega_{1}\right) \mathrm{E}_{\mathrm{z}}\left(\omega_{2}\right) \chi_{\mathrm{yyzz}}^{\mathrm{P}_{2}}\left(\frac{1}{\varepsilon_{0}-1} \ln \varepsilon_{0}-\frac{1}{\varepsilon_{0}}\right)$

Comparing this with the dipolar contributions from the interfacial molecules, (Eq.(2.3)), we have

$\frac{\chi_{\mathrm{D}, \mathrm{yyz}}^{(2)}}{\chi_{\mathrm{P}_{2}, y y z}^{(2)}} \approx \frac{<\mathrm{n}>\cdot \mathrm{N} \cdot \mathrm{a} \cdot \alpha_{\mathrm{D}}^{(2)}}{\mathrm{N} \cdot \alpha_{\mathrm{P}_{2}}^{(2)} \cdot\left(\frac{1}{\varepsilon_{0}-1} \ln \varepsilon_{0}-\frac{1}{\varepsilon_{0}}\right)} \approx \frac{1}{\left(\frac{1}{\varepsilon_{0}-1} \ln \varepsilon_{0}-\frac{1}{\varepsilon_{0}}\right)}$ 
where $\mathrm{a}$ is the typical diameter of the molecules, and $\alpha_{\mathrm{P}_{2}}^{(2)} \approx \alpha_{\mathrm{D}}^{(2)} \cdot \mathrm{a}$ is the molecular polarizability of quadrupole origin. For water interfaces, we have $\varepsilon_{0} \sim 1.8$ at optical frequencies, so Eq.(2.18) yields $\sim 5.6$. The ratio can be even larger if we consider the orientational distribution and a more reasonable molecular susceptibility model which will inevitably yield $\alpha_{\mathrm{P}_{2}}^{(2)}>\alpha_{\mathrm{D}}^{(2)} \cdot \mathrm{a}$. It suffices to say that in our simple model, we expect the dipole contribution to dominate over quadrupole contributions for the air/water interface. It can be shown that within the assumption of Eq.(2.16) similar results can be obtained for the $\chi^{Q}$ and $\chi^{M}$ terms. This simple model is also found to be effective for describing SHG from $\mathrm{C}_{60}$ thin films. ${ }^{16}$

As mentioned previously, contrary to claims in the literature, ${ }^{14}$ it is not valid to terminate the multipole expansion at a particular order. Confusion is caused by unnecessarily assuming that only one derivative is against $\mathrm{z}$ even for higher multipole contributions. To disprove the argument in ref.14, let us consider the octapole contribution with ssp polarization:

$$
P_{e f f, y}^{s}=\int_{I} \chi_{y y z z}^{P_{2}} E_{y}\left(\omega_{1}\right) \frac{d^{2} E_{z}\left(\omega_{2}\right)}{d z^{2}} d z=E_{y}\left(\omega_{1}\right) D_{z}\left(\omega_{2}\right) \int \chi_{I} \chi_{y y z z z}^{P_{2}} \frac{d^{2}}{d z^{2}}\left(\frac{1}{\varepsilon}\right) d z
$$

without making specific assumptions regarding the profile of $\varepsilon$ and $\chi_{\text {yyzze }}^{\mathrm{P}_{2}}$, Eq.(2.19) should be on the same order of magnitude as Eq.(2.15). Considerable effort has been devoted to devise a generally applicable method to separate interfacial and bulk contributions to SFG. ${ }^{17}$ The result, however, is not promising. It is probably fair to say 
that without sufficient knowledge of the molecular parameters and interfacial profile, one will have to resort to properly designed experiments to separate the bulk and interface contribution.

In conclusion, we have reviewed the theory of sum frequency generation as a surface spectroscopic technique. For a bulk medium with centrosymmetry, the second order susceptibility vanishes within the dipole approximation, and only the interfacial molecules can contribute to SFG. This unique property of SFG allows it to be explored as a valuable tool to study molecular interaction and orientation at various interfaces, including buried interfaces and liquid interfaces where only a limited number of analytical techniques exist.

\section{B. Experimental Implementation}

A typical experimental set-up is depicted in Fig. 1. Both the infrared and visible beams are incident at an angle around $45^{\circ}$. Angular separation of $5-10^{\circ}$ between the two beams is chosen so that the contribution from bulk (Eq.(2.14)) is small and there is still enough separation between SF output and reflected visible laser. The polarization of the infrared laser is controlled by a $\mathrm{ZnS}$ Fresnel romb half-wave plate, while for the visible laser the polarization is controlled by a polarizer and half-wave plate combination. A polarizer can be also inserted to control the output polarization. The infrared laser is focused to a spot size between 200 and $500 \mu \mathrm{m}$ in diameter at the sample surface, depending on the experiment. The visible laser is focused to about $500 \mu \mathrm{m}$ at the sample, with the beam waist beyond the sample position. This effectively focuses SF output after 
the sample surface where a spatial filter is placed. This spatial filter is most effective in blocking scattered visible laser light as well as the fluorescence generated in the sample by the intense visible laser. Spectral filtering by a monochrometer or a set of interference filters is also necessary. The choice between them is usually a compromise between noise reduction and signal transmission. While providing the best discrimination against fluorescence, a monochrometer can considerably reduce the detected signal (transmission $\sim 20 \%$ in the visible). A dielectric filter can have a transmittance of $10^{-5}$ for the visible laser and $80 \%$ for the SFG. Normally, two filters are enough to eliminate the scattered visible laser light. One drawback of dielectric filters is their broad transmission widow, which reduces their discrimination against fluorescence. For all our studies of water interfaces, fluorescence was found to be the most significant source of noise. While the dark current of our photomultiplier tube is about 1 count in 3000 laser shots, the fluorescence usually contributes around 1 count in 100 laser pulses even with the best spatial filtering.

A major component in any sum frequency generation set up is a tunable infrared source capable of producing high power laser pulses. In our laboratory, this is done through either optical parametric generation and amplification in nonlinear crystal $\mathrm{LiNbO}_{3}{ }^{18}$ or by difference frequency generation in $\mathrm{AgGaS}_{2}{ }^{19}$. The first set-up, on which most of the work covered in the current thesis is done, is shown in Fig. 2. Mode locked pulses from a Nd:YAG laser are used to pump the $\mathrm{LiNbO}_{3} \mathrm{OPA}_{\text {to }}$ generate tunable infrared pulses from $2.6 \mu \mathrm{m}$ to $3.6 \mu \mathrm{m}$, with energy of about $300 \mu \mathrm{J}$ per pulse, and pulse duration of about 15 psec. Details of the OPA can be found elsewhere. ${ }^{18}$ Because there is no bandwidth narrowing other than that provided by phase matching of the two matching crystals, bandwidth is quite broad, starting from about $10 \mathrm{~cm}^{-1}$ at $2800 \mathrm{~cm}^{-1}$ to $45 \mathrm{~cm}^{-1}$ at 
around $3600 \mathrm{~cm}^{-1}$. The calibration of the infrared frequency is usually accomplished by using polystyrene and mica references.

In a second set-up, shown in Fig. 3, mode locked laser pulses with energy of $40 \mathrm{~mJ}$ and pulse duration of $25 \mathrm{psec}$ from a commercial Nd:YAG laser are converted into third harmonic at $0.355 \mu \mathrm{m}$ with energy conversion efficiency of around $50 \%$. This is achieved by second harmonic and subsequently third harmonic generation in two LBO crystals. The SHG crystal is cut for type I phase matching, with non-critical phase matching at $143{ }^{\circ} \mathrm{C}$. The third harmonic generation crystal is cut for type II phase matching with angle tuning. Because the SHG crystal is too long, it converts over $85 \%$ of fundamental into SH. The optimal conversion to the third harmonic is realized when a $\lambda / 2$ wave plate is used to turn part of the input fundamental into the wrong polarization and bring it back to the right polarization (with a dual wave plate, $\lambda / 2$ for fundamental and 0 wave for SHG) after the SHG crystal for third harmonic generation. The third harmonic is then split into two beams to pump two identical but independently tunable OPAs using BBO crystals. Holographic gratings are used in both BBO OPAs to obtain narrow bandwidth tunable radiation. The output from one $\mathrm{BBO}$ OPA is then mixed with $1.06 \mu \mathrm{m}$ at a $\mathrm{AgGaS}_{2}$ crystal to generate tunable infrared radiation from 2 to $12 \mu \mathrm{m}$. The infrared energy per pulse is more than $100 \mu \mathrm{J}$ for wavelength shorter than $5 \mu \mathrm{m}$, and about $10 \mu \mathrm{J}$ at $12 \mu \mathrm{m}$. The other BBO OPA provides tunable visible laser from 0.4 to $0.8 \mu \mathrm{m}$ with energy of about $0.5 \mathrm{~mJ}$ per pulse. 


\section{References}

1. X. D. Zhu, H. Suhr, and Y. R. Shen, Phys. Rev. B 35, 3047 (1987).

2. For a recent review, see Y. R. Shen, Surf. Sci., 299, 551 (1994).

3. Y.R. Shen, Annu. Rev. Mat. Sci. 16, 69 (1986); Y.R. Shen, Nature, 337, 519 (1989); Y.R. Shen, Annu. Rev. Phys. Chem. 40, 327 (1989).

4. N. Bloembergen and P. S. Pershan, Phys. Rev. 128, 606 (1962).

5. Y. R. Shen, The Principles of Nonlinear Optics (Wiley, New York, 1984) Chapters 6, 25 .

6. P. Guyot-Sionnest, W. Chen, and Y. R. Shen, Phys. Rev. B 33, 8254 (1986); P. Guyot-Sionnest and Y. R. Shen, Phys. Rev. B 35, 4420 (1987).

7. J. A. Armstrong, N. Bloembergen, J. Ducuing, and P. S. Pershan, Phys. Rev. 127, 1918 (1962).

8. E. Adler, Phys. Rev. 134, A728 (1964).

9. Y. R. Shen, Ref. 4, p. 171.

10. G. Herzberg, Molecular Spectra and Molecular Structure, II. Infrared and Raman Spectra of Polymolecular Molecules. (Van Norstrand, New York, 1956).

11. X. D. Zhu, Ph. D. Thesis, University of California, 1989, unpublished.

12. R. G. Snyder, J. Chem. Phys., 42, 1744 (1963).

13. H. W. Schrotter and H. W. Klockner, in Raman Spectroscopy of Gases and Liquids, A. Weber, ed. (Springer-Verlag, Berlin, 1979).

14. P. Guyot-Sionnest and Y. R. Shen, Phys. Rev. B 38, 7985 (1988). 
15. P. S. Pershan, Phys. Rev. 130, 919 (1963); N. Bloembergen, R. K. Chang, S.S. Jha, and C. H. Lee, Phys. Rev. 174, 813 (1968).

16. D. Wilk, private communication.

17. R. Superfine, Ph. D. Thesis, University of California, 1991, unpublished.

18. A. Laubereau, L. Greiter and W. Kaiser, Appl. Phys. Lett. 25, 87 (1974); A. Seilmeier and W. Kaiser, Appl. Phys. 23, 113 (1980); W. Kranotzky, K. Ding, A. Seilmeier and W. Kaiser, Opt. Comm. 34, 483 (1980).

19. J. Y. Zhang, J. Y. Huang, and Y. R. Shen and C. Chen, J. Opt. Soc. Am. 10, 1758 (1993); H.-J. Krause and W. Daum, App. Phys. B 56, 8 (1993). 


\section{Figure Captions}

Fig. 1: A typical setup for surface visible infrared sum frequency generation, polarization of visible laser is controlled by a combination of a polarizer and half waveplate, polarization of infrared laser is controlled by a Fresnel rhomb which serves as a broadband half waveplate. The visible and infrared laser beams are overlaped at the sample surface both temporally and spatially.

Fig. 2: $\mathrm{LiNbO}_{3}$ optical parametric amplifier pumped by $\mathrm{Nd}: \mathrm{YAG}$ laser.

Fig. 3: BBO OPA and difference frequency generation in $\mathrm{AgGaS}_{2}$. 


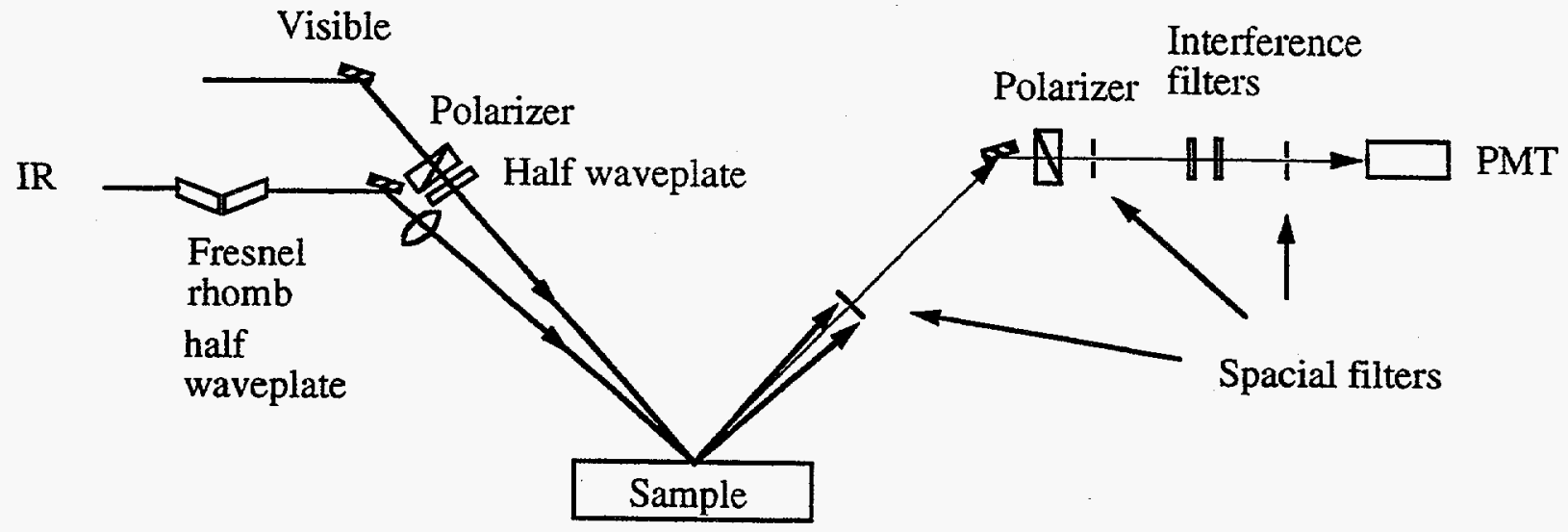

Fig. 1 


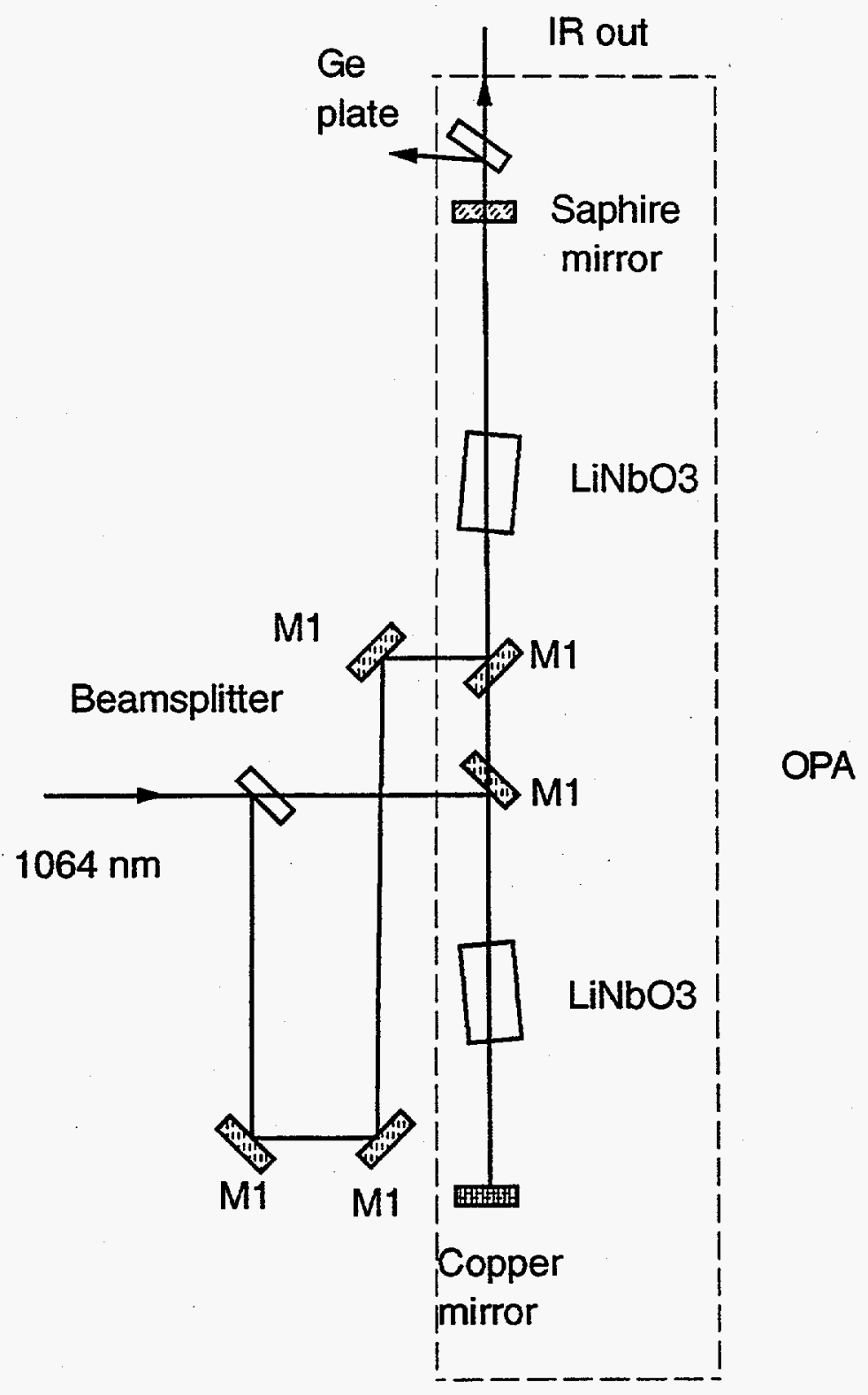

Fig. 2 


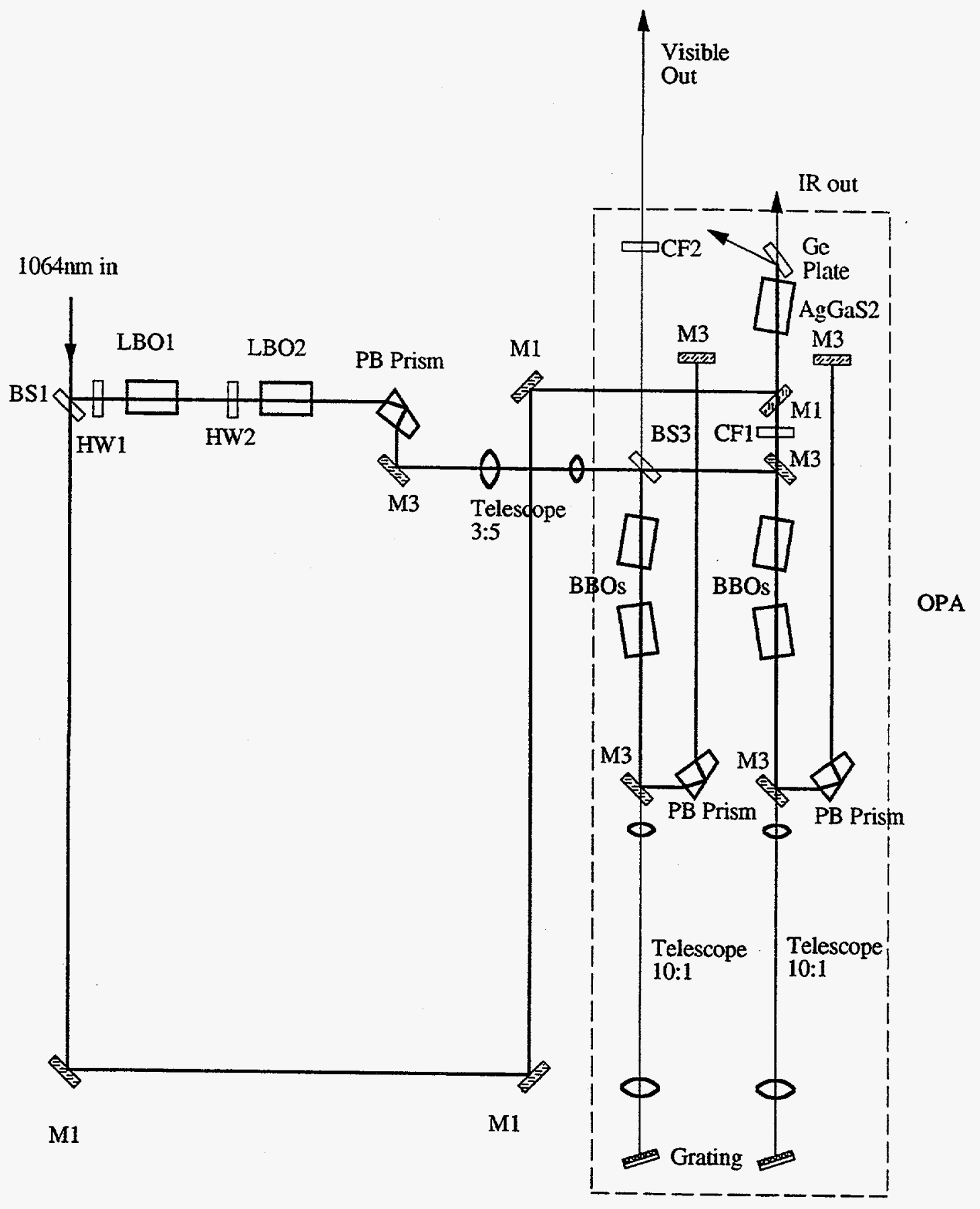

Fig. 3 


\section{Molecular Ordering at the Vapor/water Interface}

\section{A. Introduction}

The liquid/vapor interface is of fundamental interest as the transition region between two phases of a pure substance. It is also an important starting point for the understanding of heterogeneous interfaces, such as solid/liquid interfaces. To understand this kind of simple surface, a set of questions would need to be addressed, such as the density distribution across the interface, and possible difference in orientation and interaction of the molecules at the interface compared to those in the bulk. The former can be measured by X-ray reflectivity ${ }^{1}$ and in a limited sense by optical ellipsometry. ${ }^{2}$ There exists a quite reasonable agreement between theory and experiment. ${ }^{3}$ On the other hand, the orientation and interaction of molecules at the interface is less understood. This is due to the lack of analytic instruments and techniques that are available for studying liquid interfaces. It is generally believed that asymmetrical molecules should have preferred orientation at an interface. ${ }^{4}$ For alcohol surfaces, Langmuir has argued, ${ }^{5}$ based on the surface tension difference between water and alcohol, that alcohol molecules should orient to have their hydrocarbon tails pointing towards the air. This has indeed been proven to be the case for vapor /methanol interface recently by a sum frequency generation study. ${ }^{6}$ For the water surface, the picture is not as clear cut. Good ${ }^{7}$ pointed out that most hydrogenbonding liquids, including water, have characteristically lower surface entropy than other polar and non-polar liquids. He then went ahead to argue that the difference is due to the increased orientational ordering at these liquid interfaces. On the other hand, the very 
small surface potential measured ${ }^{8}$ suggests that the water molecules should have little or no polar ordering at the vapor/water interface. In a recent publication, Goh et al have used SHG to study the air/water interface, ${ }^{9}$ they concluded that the surface water molecules are polar oriented to have their oxygen atoms facing the air. However, their interpretation depends critically on the strong temperature dependence of the observed SHG signal, which suggests that the molecular orientation is strongly temperature dependent. This is not consistent with the weak temperature dependence of the surface entropy ${ }^{10}$ and surface potential. ${ }^{8}$ It turned out that the results of Ref. 9 were not reproducible, as will be discussed below. As a rule, SFG is a much more powerful technique since it can yield vibrational spectra of surface molecules that allow us to identify species and obtain detailed information on the structure and orientation of particular atomic groups within the molecules. The goal of the present study is to obtain a detailed understanding of the vapor/water interface using SFG spectroscopy, in hope to resolve the confusions regarding the orientation of water molecules at this specific interface, and also shed light on the interaction giving rise to the particular orientation. We start by reviewing the current theoretical understanding of molecular orientation at the vapor/liquid interface.

\section{B. Theory}

Because of their ubiquitous role in chemistry and biology, aqueous interfaces have long been a subject of experimental ${ }^{11}$ and theoretical interest. ${ }^{12}$ In this section we are only interested in theoretical studies that have a direct bearing on molecular orientation at vapor/liquid interfaces. Although most of the work does not directly address the vapor/water interface, it provides the basic understanding of what determines the 
orientation of molecules at the vapor/liquid interface. Considerable amount of effort has been devoted to developing a general formalism for calculating the one particle densityorientation profile across liquid interfaces, based on perturbation theory. ${ }^{13}$ The intermolecular potential is assumed to have a largely isotropic part and an anisotropic part as the perturbation, and perturbation expansion is done against the corresponding reference system with the spherical interaction. Furthermore, the interaction is usually assumed to be additive. Calculations have been carried out to investigate the important molecular property that can give rise to a preferred orientation at vapor/liquid interface. Potentials generally used are Lennard-Jones potentials $\left(V_{L, J}=4 \varepsilon\left[(\sigma / r)^{12}-(\sigma / r)^{6}\right] ; L J\right)$ centered on sites inside the molecule (each site in this case represents an atom inside the molecule), which take into account the short range repulsion due to electron cloud overlap and long range attraction due to induction and dispersion. The electrostatic interactions are accounted for with electric multipoles. For a homonuclear two-site Lennard-Jones potential (AALJ), where two identical 6-12 potentials are centered on two sites, it has been found that there is a tendency for the molecule at the liquid (high density) side of the transition region to stand perpendicular to the surface, while they lie parallel on the vapor side. This trend is reversed for the AALJ plus a point quadrupole (AALJQ) and single site LJ potential with a point dipole (SLJD). However, no polar ordering at the interface is found for all these potentials.

The simplest potential to display polar ordering is the heteronuclear Lennard-Jones model (ABLJ) where the two $L J$ potentials are different. However, when a point dipole is added to the potential (ABLJD), the polar ordering is greatly reduced. Molecules now favor to lie flat at the interface. In the electrostatic model of Stillinger, ${ }^{14}$ a single site $\mathrm{LJ}$ 
potential was used as well as electrostatic interactions from a point dipole or/and a point quadrupole moment. It was found that polar ordering requires the molecules to possess both a point dipole and a point quadrupole in the presence of a gradient of the dielectric constant. The combination of multipoles produces an asymmetry in the magnitude of electric field around the molecule, depending on the relative sign of the dipole and quadrupole moments. The molecules will then orient to immerse the high field part in the high dielectric constant region to minimize the total energy. Within this model, the dipole moment alone will not produce polar ordering since the absolute magnitude of the electric field about the molecule is symmetric with respect to inversion. In addition, lateral interaction between dipoles favors an anti-parallel orientation, so with the dipole interaction alone, the orientation will always be parallel to the surface.

The analytical treatment discussed so far is certainly quite crude, both in terms of the approximations that have to be made to derive a tractable expression and the intermolecular potentials used. An alternative is to use powerful computer simulations based on realistic potentials either derived from $a b$ initio quantum mechanical calculations, or empirically constructed by fitting with various bulk or cluster data. Over the last decade, a great deal of effort has gone into understanding the intermolecular potential of water, and there exist several standard models that are widely used and sometimes carefully compared. ${ }^{15}$ Several computer simulations studies on the vapor/water interface have appeared in recent years. ${ }^{16,17}$ Even though these studies differ widely in many computational aspects, such as the potential models of water used, the sample sizes considered and the length of trajectories analyzed, they yield, quite remarkably, a very consistent picture of the interface. Concerning the direction of the dipole moments of 
water molecules, all currently available computer simulation results indicate that the most probable orientation is parallel to the interface. However, the probability distribution is quite broad and slightly asymmetric, resulting in an excess net dipole moment from the air to the liquid. More detailed information about the structure at the surface can be obtained through analysis of the direction of the $\mathrm{OH}$ bonds at the interface. We can divide the interfacial region into two halves. On the vapor side of the interface, the most common orientation of water molecules is such that one $\mathrm{OH}$ bond points toward vapor and the other $\mathrm{OH}$ bond is directed into the liquid. In contrast, on the liquid side of the interface the $\mathrm{OH}$ bonds are most often oriented parallel to the interface with a mild tendency to point into the liquid. ${ }^{16,17}$ More discussions of the computer simulations results will be given later in this chapter as well as in chapter 5, where a detailed molecular model of the vapor/water interface will be presented.

In closing this session, it is probably fair to say that both the analytical treatment and computer simulations of the vapor/liquid interface are still in the early stages. Hydrogen bonding, long range interactions and many-body corrections such as nonadditive effects, for instance, have not been properly included. Perhaps these can explain why calculations on surface tension and surface potential have not been very reliable. ${ }^{16,17}$ Furthermore, to establish the area needing improvement as weil as the strength of the theoretical methods, experimental results capable of providing molecular level information at the interface that can be directly compared with theory is certainily in great demand. 


\section{Experimental Results}

The experimental setup has been described in chapter 2 . In the present experiment, the laser system produced $.5 \mathrm{~mJ}$ of visible pulse at $0.532 \mu \mathrm{m}$ (diameter $700 \mu \mathrm{m}$ ) and $.2 \mathrm{~mJ}$ tunable infrared pulses (diameter $300 \mu \mathrm{m}$ ) tunable from 2700 to $3900 \mathrm{~cm}^{-1}$. The bandwidth of the infrared beam varied from about $8 \mathrm{~cm}^{-1}$ around $2900 \mathrm{~cm}^{-1}$ to about $45 \mathrm{~cm}^{-1}$ at 3700 $\mathrm{cm}^{-1}$. The infrared and visible beams were incident at an angle around $50^{\circ}$ and overlapped at the vapor/liquid interface of water in a Teflon cell. The angular separation between the two laser beams was $5^{\circ}$. The cell itself sat in a copper block, and was sealed by a stainless steel top plate with a window made of infrared rated fused quartz. The whole system was temperature controlled with a commercial controller. Separate heating resistor was added to the top plate to create a temperature gradient so as to keep water from condensing on the $\mathbb{R}$ window. The Teflon cell and quartz window was cleaned thoroughly in sulfuric acid and pure water before each experiment. The stainless steel plate was cleaned in nitric acid and pure water. Triple distilled and de-ionized water (18 M $\Omega . \mathrm{cm})$ obtained from a Millipore filtration system was used. Temperature was measured with a commercial thermal couple coated with Teflon. Infrared absorption by the water vapor was kept below $10 \%$ by making the beam path in the vapor less than $3 \mathrm{~mm}$. Because the spectral width for the $\mathrm{OH}$ stretching modes is very broad, the $\mathrm{LiNbO}_{3}$ crystals need to be tuned over a wide angle. This causes a considerable beam walk of the infrared laser, so the overlap of the two beams changes significantly. To partly solve this problem, a lens system was devised so that it images the amplifier $\mathrm{LiNbO}_{3}$ crystal to the sample position. All SFG spectra were 
normalized to that from a quartz crystal. Around resonances, the signal level from the water surface was about 0.3 photons per pulse, corresponding to $\chi^{(2)} \approx$ $2 * 10^{-16} \mathrm{esu}$.

Figure 1A shows the SFG spectrum of water at the vapor/water interface for the $s s p$ polarization combination (SF output, visible input and IR input are $\mathrm{s}, \mathrm{s}$ and $\mathrm{p}$ polarized respectively). The signals with the other polarization combinations were less than $10 \%$ of the ssp peak signal and exhibited hardly any discernible resonant features within our experimental uncertainty. The spectrum in Fig. 1A can be fitted reasonably well by Eq. (2.6) assuming three vibrational resonances around 3200,3400 and $3700 \mathrm{~cm}^{-1}$. Notice that we assume Lorentzian line shapes for the resonances in Eq.(2.6). It has been argued in the literature that the bulk water spectrum $\left(3100-3600 \mathrm{~cm}^{-1}\right)$ is more accurately described by more complicated expressions. ${ }^{18}$ However, here, we will discuss only the gross features of the spectrum.

The spectrum shows two main features: a broad band between 3100 and $3500 \mathrm{~cm}^{-1}$ and a relative sharp peak at $3690 \mathrm{~cm}^{-1}$. The latter (resolution limited) can be assigned to the stretch mode of free $\mathrm{OH}^{18-20}$ The single peak above $3600 \mathrm{~cm}^{-1}$ indicates that only one $\mathrm{OH}$ of the surface water molecule can be non-hydrogen bonded. If both $\mathrm{OH}$ were free two sharp peaks would appear in the spectrum, one due to anti-symmetric mode lying above $3700 \mathrm{~cm}^{-1}$ and the other due to symmetric mode around $3600 \mathrm{~cm}^{-1}$. ${ }^{18-20}$ The resonances between 3100 and $3500 \mathrm{~cm}^{-1}$ are usually attributed to stretch modes of hydrogen-bonded $\mathrm{OH}$. 
An important issue is to determine the surface or bulk origin of the features in our SFG spectrum. For the free $\mathrm{OH}$ peak, two observations help to establish its surface origin. First, the free $\mathrm{OH}$ peak lies well outside the bulk water absorption band which is confined between 3100 and $3640 \mathrm{~cm}^{-1}$ as shown in Fig. 1(D). Second, the peak disappears upon modification of surface, as seen in the spectrum of the water surface after the deposition of monolayer of fatty alcohol, shown in Fig. 2 , which will be discussed in detail later. The surface origin of the bonded $\mathrm{OH}$ region can be established by comparing the signal at this region to that of free $\mathrm{OH}$ peak. We have argued in chapter 2 that, for the vapor/water interface, the quadrupole contribution should be much smaller than the dipole contribution. The fact that the peaks in the bonded $\mathrm{OH}$ region are of similar magnitude with free $\mathrm{OH}$ suggests that they are also due to surface dipole contribution. We will present some more evidence to support this in the following text.

We have mentioned in chapter 2 that for SFG at pure liquid interface, the presence of bulk molecules with strongly overlapping resonances can perturb the dielectric constant, and impose distortions to the spectrum through the frequency dependence of the macroscopic local field factors. Although both the frequencies of SF output and infrared laser are changing, only the local field factor of the infrared field needs to be considered, since the dielectric constant at SF has very weak dispersion. Very detailed data of dielectric constant of water in the infrared can be found in the literature (Fig.4), ${ }^{21}$ Eq. (2.10(b)) can then be used to obtain the "real" spectrum of the vapor/water interface from our measured SFG spectrum, the result is shown in Fig. 5 . Here we have let $\varepsilon_{2}=\varepsilon^{\prime}$. If we let $\varepsilon^{\prime}=1$, the distortion is less significant. Because of this ambiguity and the fact that the 
main features remain the same, we will still be referring to the raw SFG spectrum for most of our presentation.

\section{Peak Assignment of SFG Spectra}

The SFG spectrum for the vapor water interface can be understood from the infrared absorption spectra of water clusters, ${ }^{20}$ where some water molecules have nonhydrogen bonded OHs, and from the IR and Raman spectra of bulk water ${ }^{18,19}$ where both of the OHs are hydrogen bonded, at least to some extent, to neighboring molecules. ${ }^{18}$ Hydrogen bonding plays an essential role in vibrational spectroscopy of $\mathrm{OH}$ stretches of water molecules. Isolated water molecules in the vapor phase have $\mathrm{OH}$ stretch vibrations above $3600 \mathrm{~cm}^{-1}$. When a water molecule is hydrogen bonded to neighbors, its $\mathrm{OH}$ stretch vibration is shifted to lower frequency. Depending on the hydrogen bonding strength, this shift can be as large as over $400 \mathrm{~cm}^{-1}$, as in the case of ice. The intensity of $\mathrm{IR}$ absorption can be also significantly affected by the hydrogen bonding. The intramolecular coupling between the two OHs of water molecules also plays a very important role. Together with the effect due to hydrogen bonding, they command very complicated spectra for water molecules. If the two OHs are hydrogen bonded with equal strength and the molecule retains $\mathrm{C}_{2 \mathrm{v}}$ symmetry, the coupling between the two $\mathrm{OHs}$ will be most strong. The spectrum in this case can be described by two normal modes, one symmetric and one antisymmetric. In the case where the hydrogen bonding strength of the two OHs is significantly different, the spectrum can be better described as composed of two isolated local modes with different frequencies. In the situations between the two extremes, one usually still obtains two modes that are mixtures of local and normal modes. In many 
cases, intermolecular coupling has also been postulated to exist between neighboring molecules. One example is when there exists an extended hydrogen bonding network, the "isolated" symmetric mode will be coupled with the lattice motion of the molecules. The active modes for $I R$ and Raman are essentially the low frequency optical phonons near the Brillouin zone center. Another complicating factor is the strong non-uniformity of the hydrogen bonding strength in water, due to its fluid environment. The result is that the bulk spectra of liquid water is very broad, and the peak assignment in the hydrogen bonded region is extremely complicated and highly uncertain.

We begin our discussion by comparing the SFG spectrum for the vapor/water interface with the infrared absorption spectra for water clusters, ${ }^{20}$ as presented in Fig 1(BC). The qualitative resemblance between the surface and the clusters IR spectra is quite striking. Unlike liquid water, they both show a free $\mathrm{OH}$ peak at $\sim 3700 \mathrm{~cm}^{-1}$ and a broad structured band for bonded $\mathrm{OH}$ at lower frequencies. For small clusters, in which the numbers of free and bonded $\mathrm{OH}$ in the clusters are not very different, the peak heights of free and bonded $\mathrm{OH}$ modes are close. The free $\mathrm{OH}$ peak decreases relatively as the cluster size grows and the relative number of free $\mathrm{OH}$ decreases. It finally disappears in the liquid case as it should. These features reinstate our earlier conclusion that the SFG spectrum in Fig. (1A) is dominated by the $\chi_{D}^{(2)}$ contribution from the surface water molecules. This conclusion is further supported by the following observation: the free OH peak in the SFG spectrum disappears while the bonded $\mathrm{OH}$ band becomes narrower and peaks towards the low-frequency side, resembling more the IR spectrum of ice, when the water surface is covered by a monolayer of long chain alcohol molecules. This is shown in Fig. 2. 
The existence of water molecules with one free $\mathrm{OH}$ is actually not so surprising. Water molecules prefer to establish hydrogen bonds with their nearest neighbors in a tetrahedral bonding geometry. At the vapor/liquid interface, they face an environment that there are no neighboring molecules available for bonding on the vapor side. No matter how a water molecule orients itself at the interface, one hydrogen bond to neighboring molecules is necessarily broken. The situation is similar for water molecules near a large hydrophobic solute or wall. ${ }^{22}$ For the small water clusters, we can consider most of the molecules to be on the surface, so the free $\mathrm{OH}$ is of similar magnitude as the bonded $\mathrm{OH}$ peaks. As the cluster size increases, the fraction of water molecules that are on the surface decreases, so the spectral weight of free $\mathrm{OH}$ is much smaller. When the cluster size is infinite as in the bulk water, the free $\mathrm{OH}$ peak in regular infrared absorption spectrum completely disappears.

In the hydrogen bonded $\mathrm{OH}$ region, two broad peaks can be recognized, one at around $3200 \mathrm{~cm}^{-1}$ and the other one at around $3450 \mathrm{~cm}^{-1}$. The peak assignments can be made by comparing SFG spectra with the Raman spectra of bulk water. The resonant nonlinear susceptibility $\chi_{y y z}^{(2) R}$ is proportional to the isotropic component of Raman polarizability $\alpha_{y y}^{\text {Raman }}$, of which the Raman spectra have been studied extensively for bulk water. ${ }^{19}$ In the Raman spectra of bulk water, two broad peaks are observed, similar to the SFG spectra of the hydrogen bonded region. The peak at $3200 \mathrm{~cm}^{-1}$ is generally attributed to the coupled symmetric stretch mode of the tetrahedrally coordinated water molecules, since this peak dominates the infrared spectrum of ice. In the SFG spectra of the vapor/water interface, we assign this peak to the symmetric stretch mode of the surface 
water molecules with both $\mathrm{OHs}$ hydrogen bonded, with maybe one of the oxygen lone pair free and pointing away toward vapor side. The assignment of the peak at $3450 \mathrm{~cm}^{-1}$ is quite controversial. Some researchers tend to assign it to the symmetric stretch mode of asymmetrically bonded water molecules (water molecules with one hydrogen strongly bonded and the other one free or weakly bonded), while others assign it to molecules with bifurcated hydrogen bonds. ${ }^{23}$ In our case, it seems to make the best sense to assign it to the former.

\section{E. Analysis of the Free OH Peak}

We can deduce information about the free $\mathrm{OH}$ orientation from the $\mathrm{SF}$ spectra. In a simple model for $\mathrm{H}_{2} \mathrm{O},{ }^{18} \partial \mu / \partial \mathrm{Q}$ in Eq. (2.6) should be along the $\mathrm{OH}$ bond and $\partial \alpha^{(1) / \partial Q}$ has cylindrical symmetry with two components $\alpha_{\perp}$ and $\alpha_{l /}$, perpendicular and parallel to the $\mathrm{OH}$ bond. We then have only two nonvanishing SF polarizabilities $\alpha_{\perp \xi}^{(2)}$ and $\alpha_{\xi \xi \xi}^{(2)}$. Consequently, Eq. (2.3) yields,

$$
\begin{aligned}
& \chi_{D, y y z}^{R}=\frac{N_{s}}{2} \alpha\left[\left\langle\sin ^{2} \theta \cos \theta\right\rangle(1-r)+2 r\langle\cos \theta\rangle\right] \\
& \chi_{D, y y y}^{R}=\frac{N_{s}}{2} \alpha\left[\left\langle\sin ^{2} \theta \cos \theta\right)(1-r)\right]
\end{aligned}
$$

where $\alpha=\alpha_{\xi \xi \xi,}^{(2)}, \mathrm{r}=\alpha_{\perp \xi}^{(2)} / \alpha_{\xi \xi \xi}^{(2)}=\alpha_{\perp} / \alpha_{/ /}, \mathrm{N}_{\mathrm{S}}$ is the density of free $\mathrm{OH}$ at the surface and $\theta$ is the angle between the free $\mathrm{OH}$ bond and the surface normal. For free $\mathrm{OH}$, the ratio $\mathrm{r}$ has been deduced from Raman scattering to be $0.32 .{ }^{24}$ From the SFG measurements with ssp and sps polarization combinations we deduce : 


$$
\frac{\chi_{\mathrm{D}, \mathrm{yzy}}}{\chi_{\mathrm{D}, \mathrm{yyz}}} \leq 0.3
$$

Equation (5) then leads to $\theta \leq 38^{\circ}$ if a delta function orientational distribution is assumed. The latter assumption may not be valid, but the result here indicates that if we assume the $\mathrm{OH}$ bond is on average normal to the surface, the angular spread must be very significant. We can also estimate the number of free $\mathrm{OH}$ bonds on the surface, if the values of $\chi_{\mathrm{s}, \mathrm{yyz}}$ and $\alpha$ as well as the orientational averages in Eq. (3.1a) are known. By calibrating the signal at the free $\mathrm{OH}$ resonance against the signal from a quartz crystal, we obtained $\chi$ s,yyz $=2.1 * 10^{-16}$ esu. From Raman and IR. measurements we find, using Eq.(2.6), $\alpha_{\xi \xi \xi}=$ $2.5 * 10^{-30}$ esu. $^{25,26}$ The quantity in the square bracket in Eq. (3.1a) is insensitive to the $\theta$ distribution from $\theta=0^{\circ}$ to $60^{\circ}$ and yields a value of $\sim 0.65$. Equation (3.1a) then leads to $\mathrm{N}_{\mathrm{s}}=2.7^{*} 10^{14} \mathrm{~cm}^{-2}$, corresponding to $\sim 20 \%$ of a full water monolayer. This value is a lower bound; we have chosen to use the maximum value of $\alpha_{\xi \xi \xi}$ derived from Raman and IR measurements because of uncertainties in those measurements. ${ }^{27}$

We have also used an independent method to evaluate the surface density of free $\mathrm{OH}$ bonds at the vapor/water interface. We found in our measurements that the free $\mathrm{OH}$ peak in the SFG spectrum can be completely suppressed by dissolving $(11 \pm 2) \%$ of methanol in volume concentration into the bulk water. The corresponding surface density of methanol in this case is $2.5 \times 10^{14} / \mathrm{cm}^{2}$, as deduced from the result of surface tension measurement of Ref. 28. It seems reasonable to expect that each methanol molecule appearing at the vapor/water interface is hydrogen-bonded to the originally free $\mathrm{OH}$ bond. One then finds that the need of $2.5 \times 10^{14} / \mathrm{cm}^{2}$ surface methanol molecules to suppress the 
free $\mathrm{OH}$ peak in the spectrum corresponds to $25 \%$ of water molecules in a full monolayer originally having one of their $\mathrm{OH}$ bonds free, consistent with the above calculation.

The large fraction of broken hydrogen bonds on the surface can contribute significantly to the surface energy of the vapor/water interface. The average hydrogen bonding energy is $15.5 \mathrm{~kJ} / \mathrm{mol}$ for water, and the surface density can be estimated from the density of liquid. The contribution to the surface energy from the dangling $\mathrm{OH}$ bonds on $25 \%$ of the surface water molecules is then found to be $67 \mathrm{mN} / \mathrm{m}$ for air/water interface. The experimental values of surface tension and surface energy for an air/water interface at $20^{\circ} \mathrm{C}$ are 72 and $116 \mathrm{mN} / \mathrm{m}$, respectively. It shows that a reasonable calculation of surface tension of water will not be possible if the effect due to the free OHs is not properly taken into account. This might be the reason why a sophisticated calculation based on electrostatic interactions ${ }^{14}$ cannot give an even close estimate for the surface tension of water, while a very simple calculation ${ }^{29}$ gives much better result by taking into account the existence of broken hydrogen bonds on the water surface.

We can fit the spectrum of Fig.(1a) with three resonant modes in Eq. (2) and (3) (see Table 1). The amplitudes of the bonded $\mathrm{OH}$ modes appear to have a $180^{\circ}$ phase shift from that of free $\mathrm{OH}$. Since the polarizabilities for the bonded and non-bonded $\mathrm{OH}$ are most likely of the same sign, this $180^{\circ}$ phase difference indicates that the free and bonded $\mathrm{OH}$ at the interface are, on average, pointing in opposite directions. With the free $\mathrm{OH}$ pointing out of the liquid, the bonded $\mathrm{OH}$ must point into the liquid, as one should expect. This picture is consistent with the very small surface potential of water, ${ }^{8}$ which results 
from having on average, the permanent dipoles of water molecules lie close to the surface plane.

We now consider the relative peak intensity of the three peaks observed in our SFG spectrum of the vapor/water interface. To do this, we must have information about both the IR and Raman cross sections of the particular modes and the orientational distribution of the molecules. Although quantitative information of the cross sections is not available, it has been established that the cross sections of IR and Raman increase as the hydrogen bonds get stronger. In other words, they increase as the $\mathrm{OH}$ stretch

frequency decreases. ${ }^{18}$ Since the angle between the two OHs inside a water molecule is around $109^{\circ}$, the bonded $\mathrm{OH}$ of the surface water molecule will have an angle of $18^{\circ}$ from the surface plane, if the molecule has its free $\mathrm{OH}$ pointing normal to the surface. The peak at $3450 \mathrm{~cm}^{-1}$ due to the stretch mode of this bonded $\mathrm{OH}$ should then be very weak unless its angular distribution is very broad. This is probably the case for vapor/water interface. For the peak at $3200 \mathrm{~cm}^{-1}$, higher surface density and the enhancement due to symmetric coupling and strong hydrogen bonding are probably the factors that contribute to its dominance (Fig. 5) in SFG spectrum. ${ }^{18,19}$

\section{F. SFG Spectrum of Water Surface Covered by Alcohol Monolayer}

The SFG spectrum of a water surface covered by a full alcohol monolayer in Fig. 2 is also quite interesting. It has recently been shown that such a monolayer on water is able to induce an increase of the icing temperature of the nearby water because of the structure of the ordered $\mathrm{OH}$ groups in the alcohol monolayer closely matches that of a hexagonal 
ice layer in the $\underline{a b}$ plane. ${ }^{30}$ The similarity between our spectrum of the vapor/alcohol/water interface and the IR spectrum of ice, shown in Fig 2, leads us to believe that ice nucleation by the alcohol monolayer can indeed occur: the top layer of water molecules is converted into an ice-like structure which provides a seed for ice nucleation.

\section{G. Temperature Dependence of SFG Spectra of Water Surface}

We have also studied the temperature dependence of the SFG spectrum of the vapor/water interface. As shown in Fig. 3, there is no significant change over the temperature range from 10 to $80^{\circ} \mathrm{C}$. This is consistent with the observed weak temperature dependence of surface tension and surface potential of water. ${ }^{8,10}$ The only noticeable change in the spectrum is an increase of the peak around $3400 \mathrm{~cm}^{-1}$ at the expense of the peak around $3200 \mathrm{~cm}^{-1}$ when the temperature is increased, in agreement with the general trend observed in the IR and Raman spectra of bulk water. ${ }^{18,19}$ This behavior is at variance with the strong temperature dependence of the $\mathrm{OH}$ orientation predicted by some molecular dynamic simulations. ${ }^{17}$ Our result is also different from that of the SHG measurement on the vapor/water interface reported recently, ${ }^{9}$ where a substantial change of signal with temperature was observed. Because both SHG and SFG measure the polar orientation of the water molecules, it is hard to understand the large discrepancy between the two measurements. We have repeated the SHG experiment and found, within our experimental uncertainty of $\pm 10 \%$, no change in the output signal associated with $\chi_{x \geq x}^{(2)}$ from $10^{\circ} \mathrm{C}$ to $45^{\circ} \mathrm{C}$ while a $50 \%$ decrease is expected from the results of Ref. 9. This apparent contradiction was later resolved by Professor Eisenthal and his 
group. After repeating their experiment of SHG from the vapor /water interface (Ref. 9), they found that as temperature increases from $10^{\circ} \mathrm{C}$ to $80^{\circ} \mathrm{C}, \chi_{x x x}^{(2)}$ decreases by less than $10 \%$ and $\chi_{2 x}^{(2)}$ decreases by $10 \%$, consistent with our SFG and SHG measurements.

The weak dependence of the SFG and SHG on temperature can be best understood through considerations of energetics involved. Hydrogen bonding is a very strong form of intermolecular interaction. Its energy is usually smaller than that of covalent bonds, but significantly stronger than Van der Waals interaction. For water molecules, this is around $15.5 \mathrm{~kJ} / \mathrm{mol}$ per hydrogen bond. The surface water molecules must give up one hydrogen bond simply because the geometry of the molecule makes it impossible to maintain all the hydrogen bonds. The equilibrium orientation of molecules at the surface must maximize the number of hydrogen bonds at the surface, because $\mathrm{KT}$ at room temperature is only around $0.6 \mathrm{~kJ} / \mathrm{mol}$. A change of temperature of only $70{ }^{\circ} \mathrm{C}$ should certainly not change the number of free $\mathrm{OHs}$ nor significantly change the orientation of surface water molecules.

\section{H. Conclusion}

In conclusion, we have obtained for the first time a vibrational spectrum of water molecules at the vapor/water interface. The spectrum unambiguously identifies the presence of a surface species with one free $\mathrm{OH}$ projecting into the vapor. The surface density of this species is estimated to be more than $20 \%$ of a full monolayer. In addition, the spectrum suggests that roughly equal number of hydrogen bonded $\mathrm{OH}$ point into the liquid. This picture of the vapor/water interface has been predicted by molecular dynamics 
simulations and is consistent with the relatively small surface potential of water. We have also found that the SFG spectrum has no strong temperature dependence, and a monolayer of alcohol on water is able to induce an ice like structure at the interface. These results provide very useful information about surface ordering of water molecules and illustrate the promise of SFG as a tool for study of important aqueous interfaces. 


\section{References}

1. P.S. Pershan, Physica A 200, 50 (1993).

2. D. Beaglehole, in Fluid Interfacial Phenomena, C. A. Croxton Ed., (Wiley, New York, 1986), p.523.

3. J. S. Rowlison and B. Widom, Molecular Theory of Capilarity (Claredon, Oxford, 1982); Fluid Interfacial Phenomena, C. A. Croxton Ed., (Wiley, New York, 1986).

4. A. W. Adamson, Physical Chemistry of Surfaces, (Wiley, New York, 1990), p.69.

5. I. Langmuir, Colloid Symposium Monograph, (The Chemical Catalog Company, New York, 1925), p. 48.

6. R. Superfine, J. Y. Huang and Y. R. Shen, Phys. Rev. Lett. 66, 1066 (1991).

7. R. J. Good, J. Phys. Chem. 61, 810 (1957).

8. J. E. B. Randles, Phys. Chem. Liq. 7, 107 (1977); A. Borazio, J. R. Farrell and P. McTigue, J. Electroanal. Chem. 193, 103 (1985).

9. M. C. Goh et al, J. Phys. Chem. 92, 5074 (1988); M. C. Goh and K. B. Eisenthal, Chem. Phys. Lett. 157, 101 (1989)

10. W. Drost-Hansen, Ind. Eng. Chem. 57-4, 18 (1965): W. Drost-Hansen, in Chemistry and Physics of Interfaces, S. Ross, ed. ( Amer. Chem. Soc. Pulications, Washington, 1964) for a review of early work; M.A. Floriano and C.A. Angell, J. Phys. Chem., 94, 4199-4202 (1990)

11. see, for example, Water, A Comprehensive Treatise: The physics and physical chemistry of water, F. Franks, ( Plenum, New York, 1972). 
12. see W. A. Weyl, J.Coll. Sci. 6, 389 (1951) and J. Frenkel, Kinetic theory of liquids (Dover, New York, 1955) for early discussions.

13. K. E. Gubbins, in Fluid Interfacial Phenomena, C. A. Croxton Ed., (Wiley, New York, 1986), p.469.

14. F. H. Stillinger, Jr. And A. Ben Naim, J. Chem. Phys. 47, 4431 (1967).

15. For a recent review see K. Watanabe and M. L. Klein, Chem. Phys. 131, 157 (1989).

16. R. M. Townsend and S. A Rice, J. Chem. Phys. 94, 2207 (1991); M.A. Wilson, A. Pohorille and L.R. Pratt, J. Phys. Chem. 91,4873 (1987); A. Pohorille and M. A. Wilson, J. Molecular Structure 284, 271 (1993) and references therein.

17. M. Matsumoto and Y. Kataoka, J. Chem. Phys. 88, 3233 (1988).

18. J. R. Scherer, in Advances in Infrared and Raman Spectroscopy, Vol. 5 pp. 149, edited R. J. H. Clark and R. E. Hester ( Heyden, Philadelphia, 1978 ).

19. G. E. Walrafen, M. S. Hokmabadi and W. H. Yang, J. Chem. Phys. 85, 6964 (1986); D. E. Hare and C. M. Sorensen, J. Chem. Phys. 93, 25 (1990).

20. R. H. Page, J. G. Frey, Y. R. Shen and Y. T. Lee, Chem. Phys. Lett. 106, 373 (1984); D. F. Coker, R. E. Miller and R. O. Watts, J. Chem. Phys. 82, 3554 (1985).

21. H. D. Downing and D. Williams, J. Geophys. Res. 80, 1656 (1975).

22. J. N. Israelachvili, Intermolecular and Surface Forces (Academic Press, London, 1989), p 102; L. P. Pratt and D. Chandler, J. Chem. Phys. 67, 3683 (1977).

23. P. A. Gignere, J. Raman Spec. 15 (5), 354 (1984).

24. W.F. Murphy, Mol. Phys. 36, 727 (1978). 
25. W. Hagen, A. G. M. Tielens and J. M. Greenberg, Chem. Phys. 56, 367 (1981); W. B. Person, NATO ASI Matrix Isolation Spectroscopy, Montpelier (1980); M. Gussoni, J. Mol. St. 141, 63 (1986), S. A. Clough, Y. Beers, G. P. Klein, and L.s. Rotman, J. Chem. Phys. 59, 2554 (1973).

26. We have used the value of the bond polarizability from Ref.[24] rescaled by the larger absolute differential Raman cross section measured by N. Abe and M. Ito, J. Raman Spect. 7, 161 (1978) to obtain an upper bound of $\alpha_{\xi \xi \xi .}$

27. From the bonding picture of the surface monolayer, one expects that the fraction of surface water molecules that possessing a free $\mathrm{OH}$ bond cannot be more than $50 \%$.

28. J. J. Kipling, J. Colloid Sci. 18, 502 (1963).

29. A. Luzar, Chem. Phys. Lett. 96, 485 (1983).

30. M. Gavish, Ronit Popovitz-Biro, Meir Lahav, Leslie Leisorowitz, Science 250, 973 (1990) 


\section{Figure Captions}

Fig. 1:A) SFG spectrum of the pure water/vapor interface at $40^{\circ} \mathrm{C}$ with polarizations combination s, s, and $\mathrm{p}$ for the sum-frequency, visible, and infrared beams, respectively. Solid line is a theoretical fit using Eq.(3). B-C) Infrared spectra of water clusters with sizes indicated ( From Ref. 20). D) Infrared absoption spectrum of liquid water ( from E. Whalrafen, J. Chem. Phys. 47, 114 (1967)).

Fig. 2: SFG spectrum (dotted line), with the ssp polarization combination, of water surface covered by a stearyl alcohol monolayer compressed to $10 \mathrm{mN} / \mathrm{m}$. For comparison, the SFG spectrum of pure water surface (dashed line) and the infrared absorption spectrum of ice (solid line) ( from E. Whallhey and J.E. Bertie, J. Chem. Phys. 46, 1264 (1967)) are also shown.

Fig. 3: SFG spectra, with the ssp polarization combination, of a pure water/vapor interface at four temperatures.

Fig. 4: The (a) imaginary and (b) real part of refrative index of bulk water in the infrared (From Ref. 21).

Fig. 5: SFG spectrum of vapor/water interface with ssp polarization at $40^{\circ} \mathrm{C}$, corrected for macroscopic local field factor of infrared field. 
Table 1: Parameters obtained by fitting the SFG spectrum of Fig. 1A with three resonant modes using Eq. (3)

\begin{tabular}{|c|c|c|}
\hline$\omega_{\mathrm{q}}\left(\mathrm{cm}^{-1}\right)$ & $\Gamma_{\mathrm{q}}\left(\mathrm{cm}^{-1}\right)$ & $\mathrm{A}_{\mathrm{q}}$ (a.u.) \\
\hline 3257.5 & 108.6 & 24.2 \\
\hline 3451.3 & 101.5 & 76.4 \\
\hline 3682.9 & 33.3 & -38.6 \\
\hline
\end{tabular}

$$
\chi_{\mathrm{NR}}=-0.4
$$



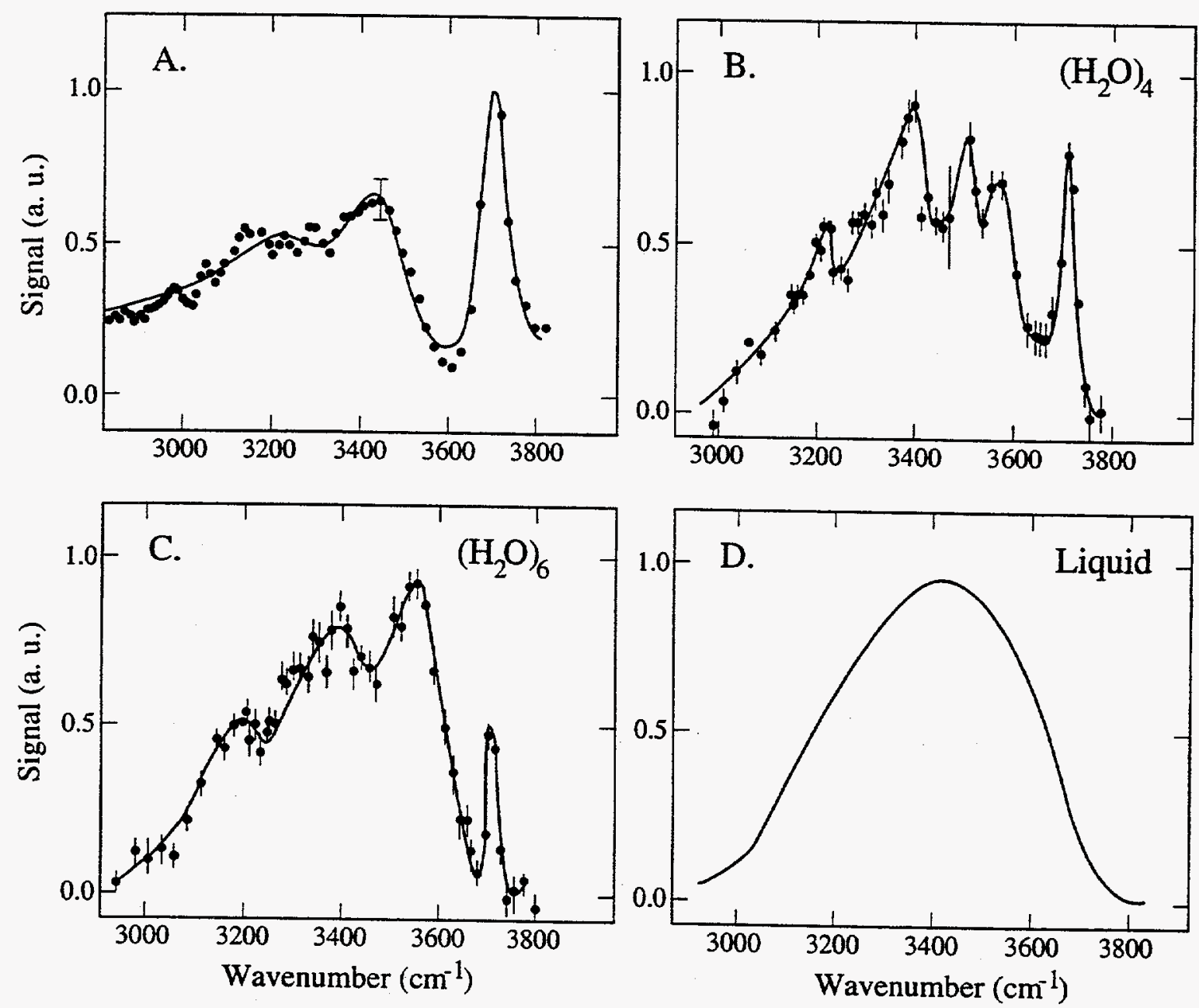

XBL $9211-4774$

Fig. 1 


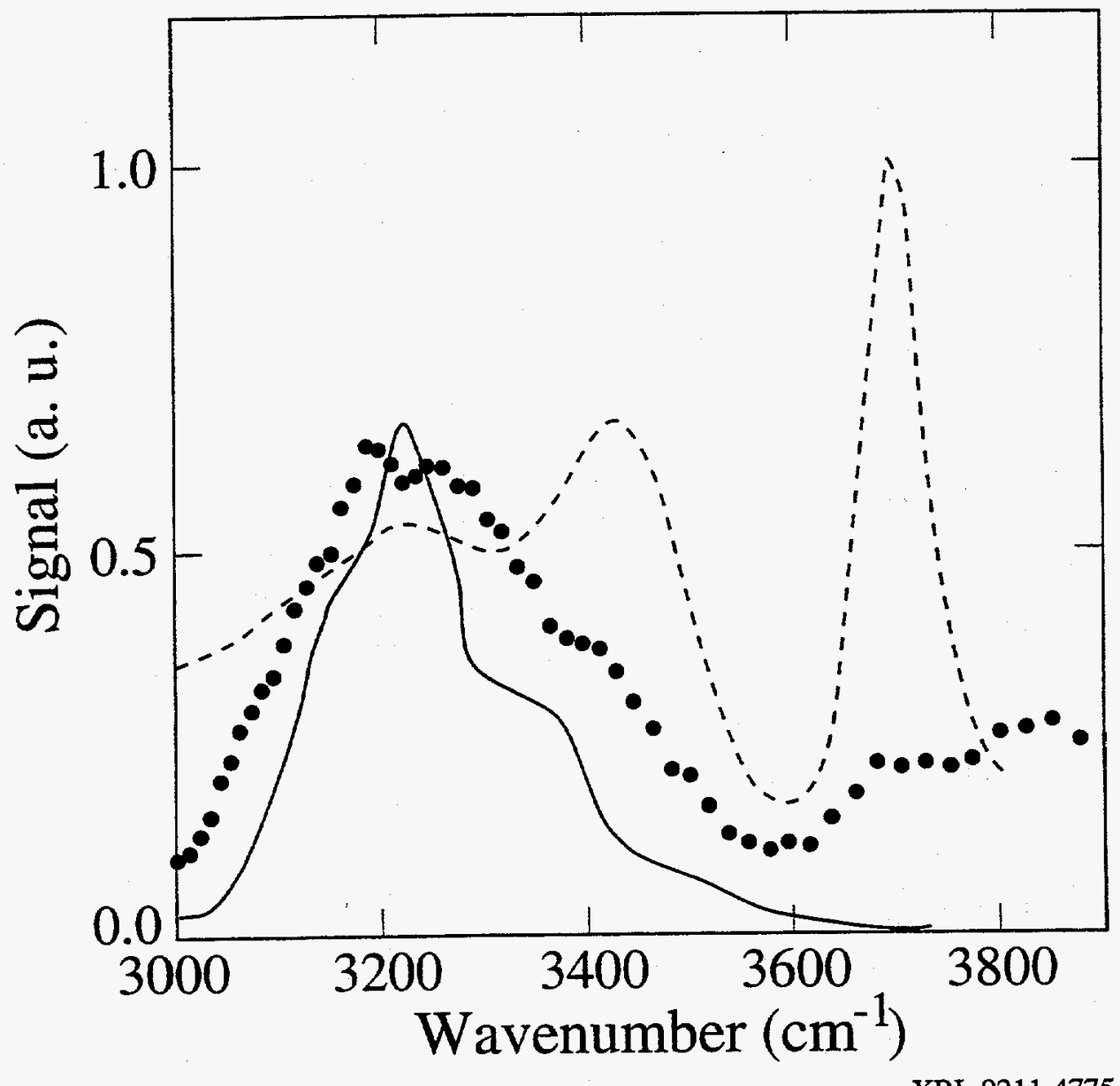

XBL 9211-4775

Fig. 2 


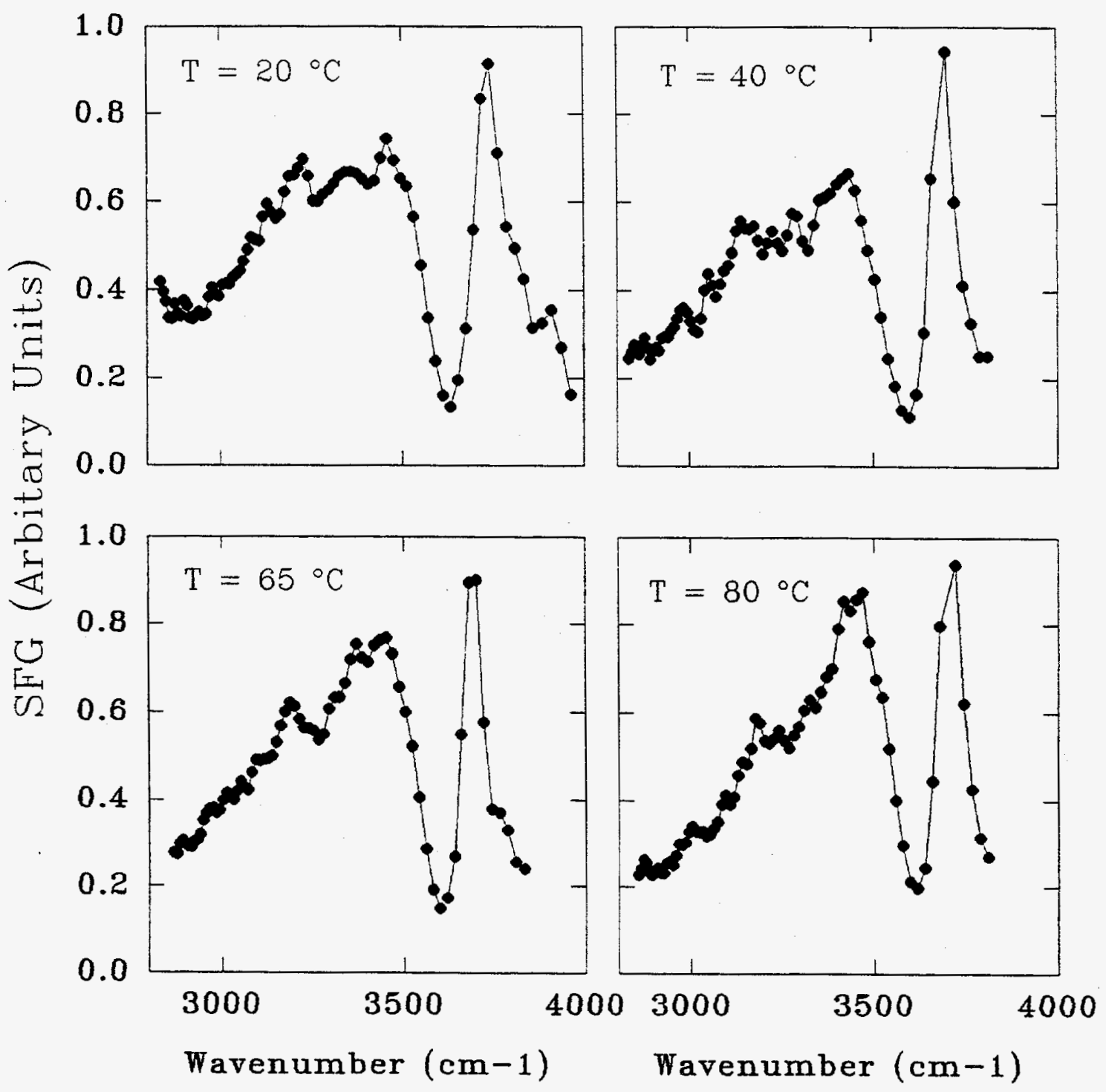

Fig. 3 

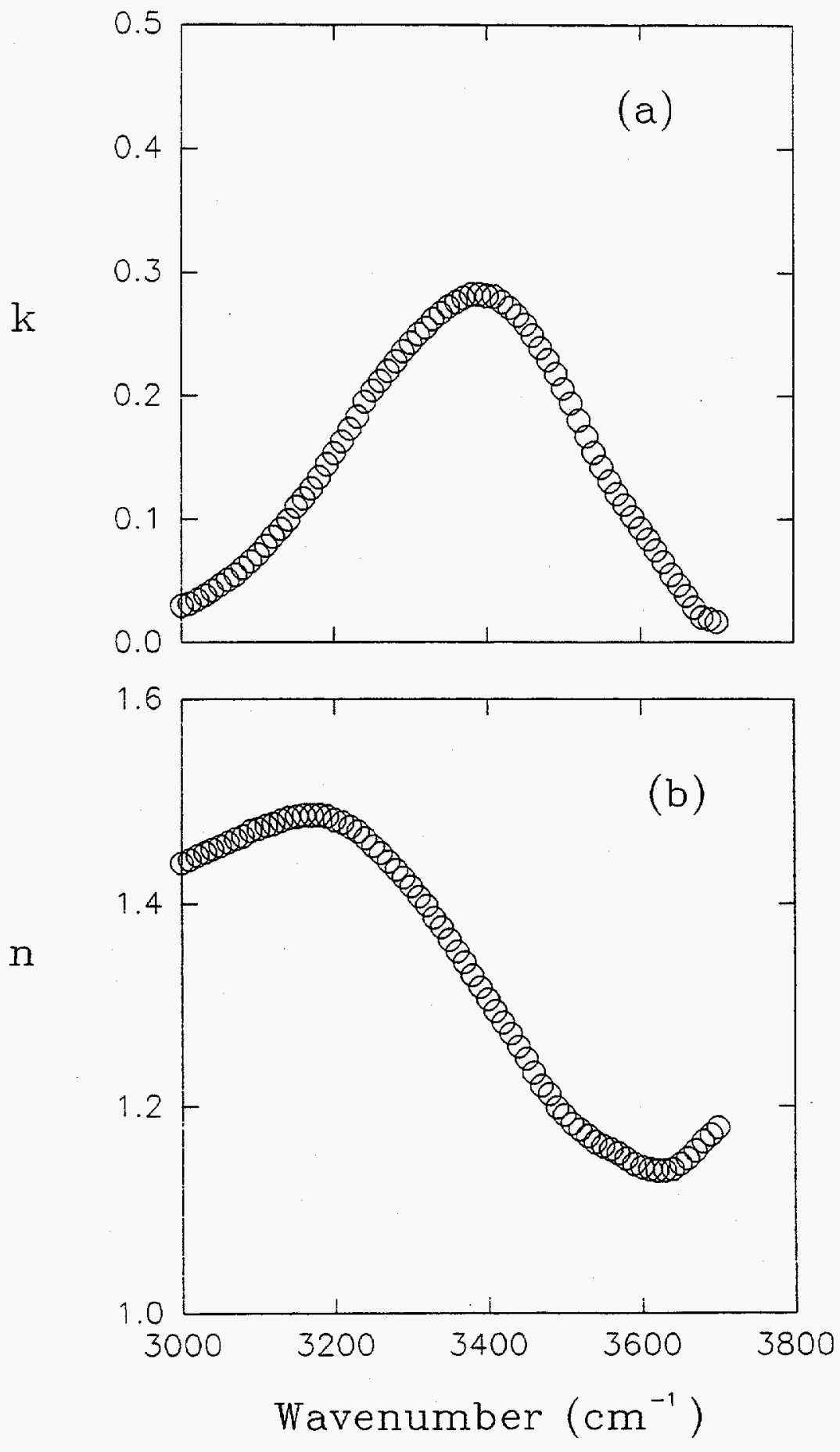

Fig. 4 


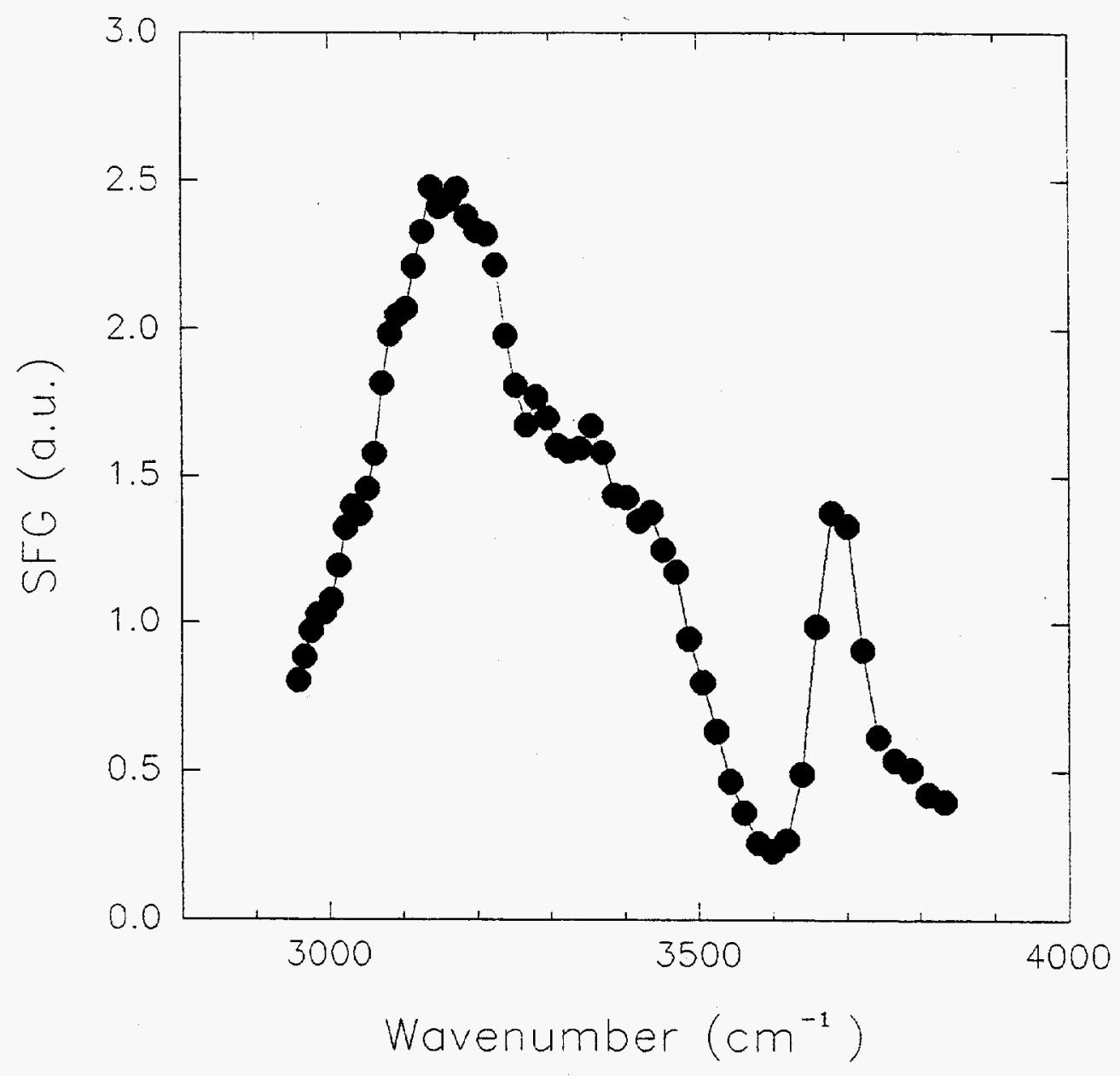

Fig. 5 


\section{Interaction of Water Molecules with Silica Surface}

\section{A. Introduction}

Buried water interfaces play an important role in many surface science phenomena. Examples range from electrochemistry, ${ }^{1}$ micelle formation, ${ }^{2}$ to biological processes such as membrane stability ${ }^{2}$ and protein activity. ${ }^{3}$ Over the past few decades, a great deal of insight on interfacial water molecules has been gained from theoretical studies, especially those employing numerical simulations. ${ }^{4}$ Experimental research on the topic, however, has been limited. Measurements of the forces between two surfaces immersed in aqueous solution, but separated at molecular distances, seem to indicate that water molecules at the surfaces have both enhanced translational and orientational order compared to the bulk. ${ }^{5}$ Nuclear magnetic resonance studies also show some evidence that water molecules at surfaces behave differently than in the bulk. ${ }^{6}$ Optical second harmonic generation (SHG) and sum-frequency generation (SFG) have recently been proven to be ideal tools for investigation of liquid interfaces. ${ }^{7-9}$ They can also be readily adapted to probe water/solid interfaces. Indeed, a SHG study has already been carried out, illustrating that water molecules near a charged surface are strongly oriented. ${ }^{8}$ Unfortunately, little additional detail could be deduced due to the lack of spectroscopic information.

In this chapter, we present our work on the vibrational spectra of water molecules, obtained by infrared-visible SFG, at fused quartz/water interfaces under various conditions. The results indicate that water molecules can interact with a quartz surface via two opposing forces: hydrogen bonding with silanol groups on the quartz surface and 
electrostatic interaction with surface charges resulting from ionization of the surface silanol groups. By varying the degree of surface ionization through adjustment of the $\mathrm{pH}$ value of the bulk water, it is possible to alter the relative strength of the two opposing forces, and induce a flip in the orientation of the surface water molecules. It appears that the orientations of the interfacial water molecules are closely related to the hydration force and heterogeneous ice nucleation at interfaces. A study of fatty acid monolayers on water surfaces suggest that this general behavior can be found at other water interfaces.

\section{B. Theory and Experiment}

SFG as a technique for surface vibrational spectroscopy has been described in earlier publications. ${ }^{7}$ Within the electric dipole approximation, the SFG process is forbidden in a centrosymmetric bulk medium like water, but allowed at the surface where the inversion symmetry is broken. It has been established in a previous paper ${ }^{9}$ that the quadrupolar bulk contribution from water is not appreciable and the SFG is mainly from the dipolar contribution of water molecules with preferred orientation at the surface. The reflected SFG signal is proportional to the square of the surface nonlinear susceptibility $\chi_{\mathrm{s}}^{(2)}\left(\omega_{\mathrm{sf}}=\omega_{1}+\omega_{2}\right)$, which can be decomposed into a resonant and a nonresonant part,

$$
\chi_{s}^{(2)}=\chi_{s}^{(2) R}+\chi_{s}^{(2) N R}
$$


with $\omega_{2}$ near resonance and $\omega_{1}$ off resonance. The resonant $\chi_{s}^{(2) R}$ can be related to the second order molecular polarizability $\alpha_{\operatorname{lm} n}^{(2) R}$ through a coordinate transformation averaged over the molecular orientational distribution, denoted below by the angular brackets,

$\chi_{s, j \mathrm{jk}}^{(2) R}=N_{s} \cdot<(\hat{\mathrm{i}} \cdot \hat{\mathrm{l}})(\hat{\mathrm{j}} \cdot \hat{\mathrm{m}})(\hat{\mathrm{k}} \cdot \hat{\mathrm{n}})>\alpha_{\mathrm{lmn}}^{(2) \mathrm{R}}$

$\alpha_{\operatorname{lmn}}^{(2) R}=\sum_{q} \frac{A_{q}}{\left(\omega_{q}-\omega_{2}-i \Gamma_{q}\right)} \Delta \rho_{g q}$

where $\mathrm{N}_{\mathrm{S}}$ is the number of molecules per unit area contributing to the orientational average, while $A_{q}, \omega_{q}$, and $\Gamma_{q}$ are the strength, resonant frequency, and damping constants of the qth vibrational mode of the molecules. The SFG spectrum is obtained by tuning $\omega_{2}$ over molecular vibrational resonances. In our experiment on quartz/water interfaces, the fused quartz plate may also contribute to $\chi_{s}^{(2)}$. However, this contribution is small and non resonant, and can thus be simply absorbed into $\chi_{\mathrm{s}}^{(2) \mathrm{NR}}$. We also did not find spectral features due to the silanol ( $\mathrm{SiOH})$ groups at the quartz surface. Their contribution is expected to be small because the density of the surface silanol groups is known to be about $5 / \mathrm{nm}^{2},{ }^{10}$ which is smaller than the surface density of $\mathrm{OH}$ groups of $20 / \mathrm{nm}^{2}$ from water molecules at the surface. Furthermore, the bond angle between $\mathrm{Si}-\mathrm{O}$ and $\mathrm{O}-\mathrm{H}$ is about $120^{\circ}$; with the $\mathrm{Si}-\mathrm{O}$ bond arranged perpendicular to the surface, the $\mathrm{O}-\mathrm{H}$ bond is tilted at $60^{\circ}$ from the surface normal, making it weak for SFG detection.

The experimental setup for our SFG measurement has also been described elsewhere. ${ }^{7}$ Briefly, a picosecond passive-active mode-locked laser system produced 0.5 $\mathrm{mJ}$ visible pulses at $0.532 \mu \mathrm{m}$ and $0.2 \mathrm{~mJ}$ infrared pulses tunable from $2700 \mathrm{~cm}^{-1}$ to 3900 
$\mathrm{cm}^{-1}$. Both pulses were incident at angles of about $47^{\circ}$ from the quartz (fused) side and overlapped at the quartz/water interface formed by a fused quartz window in contact with ultra pure water (resistivity higher than $18 \mathrm{M} \Omega . \mathrm{cm}$, obtained from Millipore filtration system) in a Teflon cell. Careful cleaning of the window and the cell was necessary to avoid any contamination. To vary the $\mathrm{pH}$ value of the bulk water, Fisher standard $\mathrm{HCl}$ and $\mathrm{NaOH}$ solutions were used. Adding $\mathrm{NaCl}$ to the solution allowed control of the electrolyte concentration. All SFG spectra were normalized to the SFG signal reflected from a bare quartz crystal in order to reduce the effects of laser fluctuation, change of beam overlap, and $\mathbb{R}$ absorption ( $10 \%)$ of the fused quartz window.

Cleaning of the fused quartz window deserves some additional explanation, although it may appear straightforward. A silica (including fused quartz) surface can be either hydrophilic or hydrophobic, depending on the number of silanol groups at the surface. The hydroxylation and dehydroxylation of silica surface has been studied extensively in the past, as the degree of hydroxylation controls the surface chemistry of silica ${ }^{11}$ to a large extent. A fully hydroxylated silica surface has about 5 silanol groups $/ \mathrm{nm}^{2}$, and appears hydrophilic. When heated to $\sim 1000^{\circ} \mathrm{C}$ in vacuum, all the silanol groups are converted to siloxane groups, and the surface appears hydrophobic. Rehydroxylation time is about 5 years if the silica is kept in contact with water at room temperature. If the silica is boiled in water, this process can be speeded up to 60 hours. In our experiments, it was found that cleaning the fused quartz windows in highly oxidizing sulfuric acid solutions, followed by heavy rinsing and soaking in ultra pure water can get rid of most impurities. However, our SFG spectra did not stabilize until many cycles of such cleaning were done. 
It is possible that the window plates we used were not fully hydroxylated, and only after many cycles of cleaning does the surface becomes fully hydroxylated.

To determine the phase of the surface susceptibility $\chi_{s}^{(2)}$, an interference measurement was set up. ${ }^{12}$ As shown in Fig. 1, the three fields - the reflected visible and infrared, and the generated sum frequency fields - are redirected to and overlapped at a nonlinear optical crystal (crystalline quartz in our case) by a calcium fluoride lens, after going through a home built phase compensator. It is easy to see that the total sum frequency generation depends on the interference between the SFG at the sample surface and at the nonlinear crystal, with the relative phase shift controlled by the phase compensator. Experimentally, the best contrast can be obtained by optimal overlap of the three beams at the quartz crystal, with the relative amplitude controlled by the orientation of the quartz crystal. An output polarizer is also needed to achieve optimum contrast.

\section{SFG Spectra and Peak Assignment}

Figure 2 presents the SFG spectra obtained from fused quartz/water interfaces for different $\mathrm{pH}$ values of the bulk water. A spectrum of the quartz/ice interface is also included for comparison. The output/input polarization combination for the spectra is soutput, s-visible input and p-infrared input, respectively. The ssp spectra are chosen to be discussed here because the resonant surface nonlinear susceptibility component $\chi_{y y z}^{(2) R}$ (with $\hat{z}$ along the surface normal) that can be deduced from the spectra is proportional to the Raman polarizability $\alpha_{y y}^{R a m a n}$ of which the bulk water spectra have been discussed extensively in the literature. Indeed, our spectra resemble those of the isotropic Raman 
component of liquid water, ${ }^{13}$ except that the peaks in our spectra are narrower, presumably due to the restricted motion of water molecules at the surface. Two characteristic peaks appear at about $3200 \mathrm{~cm}^{-1}$ and $3450 \mathrm{~cm}^{-1}$, respectively, with another barely visible one at $3600 \mathrm{~cm}^{-1}$. The peak at $3200 \mathrm{~cm}^{-1}$ is generally attributed to the coupled symmetric $\mathrm{OH}$ stretch mode of tetrahedraly coordinated water molecules. ${ }^{13(b)}$ This peak dominates in the ice spectra, therefore its strength is an indication of bond ordering in the water molecular arrangement. The assignment of the peak at $3450 \mathrm{~cm}^{-1}$ is still controversial. Some researchers assign it to the symmetric stretch mode of asymmetrically bonded water molecules ${ }^{13(b)}$ (molecules with one $\mathrm{H}$ strongly hydrogen bonded and the other $\mathrm{H}$ weakly bonded to neighboring molecules). Others assign it to molecules with bifurcated hydrogen bonds. ${ }^{13(c)}$ We believe that the first assignment is more suitable, since quite some criticism has been raised about the existence of the bifurcated hydrogen bonds. The strength of the peak at $3450 \mathrm{~cm}^{-1}$ is then an indication of bond disordering in the water molecular arrangement. The peak at $3600 \mathrm{~cm}^{-1}$ is believed to be due to antisymmetric $\mathrm{OH}$ stretch of asymmetrically bonded water molecules. This peak is never prominent in our spectra. Its extreme weakness presumably results from certain degree of forbiddance in the corresponding Raman scattering. This then suggests by inference that the anti-symmetric $\mathrm{OH}$ stretch of the tetrahedraly coordinated water molecules is also not easily detectable, as it is missing in our spectra.

\section{D. pH Dependence of the SFG Spectra}

The spectra in Fig. 2 show how the two peaks at 3200 and $3450 \mathrm{~cm}^{-1}$ vary with the $\mathrm{pH}$ value of the water. At $\mathrm{pH}=1.5$, the spectrum in Fig. 2 (a) resembles that of the 
quartz/ice interface in Fig. 2 (f), indicating that most of the interfacial water molecules are ordered, with tetrahedral coordination. As the $\mathrm{pH}$ value increases (Fig. 2 (b) - Fig. 2 (e)), the relative strength of the two peaks varies. The $3200 \mathrm{~cm}^{-1}$ peak first decreases while the $3450 \mathrm{~cm}^{-1}$ peak increases. Then, with $\mathrm{pH}>5.6$, both peaks strengthen, with the former increasing more rapidly. Finally, at $\mathrm{pH} \sim 12.3$, the spectrum of Fig. 2 (e) again resembles that of the quartz/ice interface, but the peak strength is significantly larger than in the latter case as well as in Fig. 2 (a). The results can be understood from possible surface induced ordering or disordering of the interfacial water molecules.

The surface chemistry of silica has been well studied. ${ }^{14}$ The surface $\mathrm{OH}$ groups (silanol groups) can be negatively charged by deprotonation in water with a sufficiently low proton concentration (high $\mathrm{pH}$ values). An important parameter for aqueous oxide interface is $\mathrm{pH}$ at which the surface is neutral, called the point of zero charge (pzc). For a quartz surface, the pzc is around $\mathrm{pH} 2$. A quartz surface is neutral for $\mathrm{pH} \sim 2$ and fully ionized for $\mathrm{pH} \geq 10 .{ }^{15}$ With the surface silanol groups completely ionized, the negative surface charges should produce a strong electric field of $\sim 10^{7} \mathrm{~V} / \mathrm{cm}$ close to the surface, taking dielectric saturation into account. ${ }^{16}$ This field can orient several layers of interfacial water molecules with their dipole moments pointing towards the solid. The similarity between the spectrum of quartz/water at $\mathrm{pH} \sim 12$ (Fig. 2(e)) and quartz/ice interfaces (Fig. $2(\mathrm{f})$ ), suggests that the interfacial structure of water at an electrified surface is icelike. This is consistent with the general notion that an electric field can nucleate ice, ${ }^{17}$ but contrary to some molecular dynamics simulations which indicate that water molecules close to a charged surface may not be as well hydrogen-bonded as ice. ${ }^{18}$ 
If the quartz surface is neutral (e.g., at $\mathrm{pH}=1.5$ ), the interfacial water molecules tend to form hydrogen bonds with their oxygen facing the quartz surface, ${ }^{19}$ opposite to the orientation expected for the high $\mathrm{pH}$ case. The spectrum of Fig. 2 (a) shows that the structure is also ice-like. However, compared to the high $\mathrm{pH}$ case, the signal strength is smaller, by a factor of $\sim 7$. With hydrogen bonding to the surface providing the orienting force, one would expect that only one or two monolayers of water molecules would be oriented. This then suggests that for the high $\mathrm{pH}$ case, the surface field can align up to 3 to 5 layers of interfacial water molecules. Measurements of the force between two charged ionic surfaces immersed in water found a strong repulsive force that extended over many molecular layers. ${ }^{5(a)}$ It was latter suggested that this force is due to polar oriented water molecules close to the charged surfaces. ${ }^{5(b)}$ The existence of polar ordered water molecules is direct evidence in support of this theory. In addition, the range that the polar ordering of surface water molecules extends is consistent with the range of the observed repulsive force.

For an intermediate pH value (Fig. 2 (b) or (c)), the spectrum is weaker and the two peaks at 3200 and $3450 \mathrm{~cm}^{-1}$ appear equally pronounced, indicating a reduction of the ordering of the interfacial water molecular arrangement. This is also supported by the appearance of the weak shoulder at about $3600 \mathrm{~cm}^{-1}$. The relative strength of the two major peaks varies significantly with the $\mathrm{pH}$ value (Fig. 2 (b)-(d)), suggesting that the ordering of molecular arrangement can be readily perturbed by the degree of surface ionization. Our understanding of the results is as follows: when the surface is partially ionized, opposing alignment forces acting on interfacial molecules from the surface field and from hydrogen bonding with the surface silanol groups compete with each other and 
yield a less ordered interfacial water structure. Because the surface field has an orienting effect over several molecular monolayers, the spectral intensity increases as the surface field becomes more dominant (Fig. 2 (d)). This then suggests that the repulsive hydration force from strong ionic surfaces extends over a longer range than that from surfaces terminated only by polar groups. ${ }^{20}$

To see whether the water molecules at the quartz/water interfaces indeed have opposite orientations at low and high $\mathrm{pH}$ values, we have measured the phases of $\chi_{\mathrm{yyz}}^{(2) \mathrm{R}}$ for the $3200 \mathrm{~cm}^{-1}$ peak at low and high $\mathrm{pH}$ values. The results are shown in Fig. 5 . As we can see, the surface susceptibilities for low $\mathrm{pH}$ and high $\mathrm{pH}$ are $180^{\circ}$ out of phase with each other, indicating that the molecular orientations are reversed.

To further confirm this picture, we have studied the effect of electrolyte on the SFG spectra of quartz/water interfaces by dissolving $\mathrm{NaCl}$ in the aqueous solution. Representative results are shown in Fig. 3. For the low pH case (Fig. 3 (a)) with a neutral quartz surface, the salt ions (even at $0.5 \mathrm{M} \mathrm{NaCl}$ ) have no effect on the spectrum as they are not expected to appreciably disturb the interfacial system. For the high $\mathrm{pH}$ case (Fig. 3(c)), the addition of $\mathrm{NaCl}$ reduces the spectral intensity because of the screening of the surface field by the positive $\mathrm{Na}^{+}$ions. However, it is interesting to note that the spectrum actually indicates an increase in molecular ordering. This is because ions now participate in the formation of a thinner double layer, leading to an enhancement of the double-layer surface field. For the pure water case (Fig. 3 (b)), the effect of the electrolyte is much more pronounced because the balance of the two competing forces at the interface is 
easily upset by the presence of $\mathrm{Na}^{+}$ions; the spectrum now resembles that of the low $\mathrm{pH}$ case.

We now discuss the SFG spectra for quartz/ice interfaces. The interfaces were prepared by gradually cooling the fused quartz window using cold nitrogen gas to around $-10{ }^{\circ} \mathrm{C}$ over several hours, before freezing suddenly set in at the quartz/water interface. Comparison between the quartz/ice and quartz/water interface spectra, Fig. 2 (f) and Fig. 2 (a), shows that the two have roughly the same strength, both arising from approximately one monolayer of surface water molecules. The stronger dominance of the $3200 \mathrm{~cm}^{-1}$ peak in the quartz/ice interface spectrum indicates a better bond ordering of the ice surface, as is expected. Spectra of quartz/ice interfaces frozen from bulk water with different $\mathrm{pH}$ values do not show appreciable difference in their spectral features and overall strengths. Measurements of the phase of the $3200 \mathrm{~cm}^{-1}$ peak lead to the conclusion that during freezing, the interfacial water molecules contributing to the spectrum do not flip in their orientation. In the high $\mathrm{pH}$ case, the sudden decrease of the signal strength upon freezing must be due to reversal of the inner field-oriented layers of interfacial water molecules to non polar structure. This suggests that the structure of polar ordered water molecules is not compatible with the structure of hexagonal ice (Ih). We notice that for cubic ice structure (Ic), it is possible to arrange water molecules with their dipoles all along the same direction without sacrificing any hydrogen bonding, but it is not so for ice in the usual Ih form. For liquid water in a very strong field, one may think that molecules tend to approach the structure of Ic in order to lower the overall free energy. 


\section{E. Polarization Dependence of the SFG Spectra}

We have also obtained the SFG spectra of quartz/water interfaces with different input/output polarization combinations, shown in Fig. 4 for three different $\mathrm{pH}$ values. Unlike the case of vapor/water interfaces reported in Ref. 9, the spectra here for all polarization combinations are clearly visible, suggesting that the orientaional symmetry of the interfacial water molecules is probably lower than in the former case. For the pss and sps polarization combinations, which yield $\chi_{y y z}^{(2)}$ and $\chi_{y z y}^{(2)}$, respectively, the spectra seem to be less sensitive to bond ordering than the ssp spectrum. As $\mathrm{pH}$ is varied, the $3450 \mathrm{~cm}^{-1}$ peak remains at least as prominent as the $3600 \mathrm{~cm}^{-1}$ peak. Their relative strength also correlates well with disordering in the interfacial water structure.

The spectra in Fig. 4 can, in principle, allow us to deduce some quantitative information about the average $\mathrm{OH}$ bond orientation, assuming that the peak assignment is definite. In reality, the analysis is quite complicated and may not always guarantee a clean result. Let us consider the peak at $3200 \mathrm{~cm}^{-1}$. We assume that the hydrogen bonds of the two $\mathrm{OHs}$ of the water molecules are equally strong, and that the peak is from the symmetric stretch mode of these water molecules alone. To reduce the number of adjustable variables, we assume that the bond polarizabilities are additive. The nonvanishing molecular polarizabilities can be expressed as follows,

$$
\begin{aligned}
& \alpha_{\mathrm{acc}}^{\mathrm{R}}=-\frac{1}{2}[(1+r)-(1-r) \cos \alpha] \cos \left(\frac{\alpha}{2}\right) \\
& \alpha_{\mathrm{bbc}}^{\mathrm{R}}=-\frac{r}{2} \cos \left(\frac{\alpha}{2}\right) \\
& \alpha_{\mathrm{ccc}}^{\mathrm{R}}=-\frac{1}{2}[(1+r)+(1-r) \cos \alpha] \cos \left(\frac{\alpha}{2}\right)
\end{aligned}
$$


here we assume that IR transition moment is along the $\mathrm{OH}$ bond direction, and that the Raman polarizability of individual $\mathrm{OH}$ bonds have components both along the bond direction and perpendicular to the bond direction, with the ratio between them being r. $\alpha$ is the $\mathrm{HOH}$ angle, and ac forms the plane of the molecule with $\mathrm{c}$ along the bisector of the two $\mathrm{OH}$ bonds, and $\mathrm{b}$ perpendicular to the plane. The surface susceptibility can then be derived,

$$
\begin{aligned}
\chi_{y y z s}^{(2)}= & \frac{N_{s}}{2}\left[<\sin ^{2} \theta \cos \theta>\alpha_{c c c}^{R}+<\cos ^{2} \varphi \cos \theta+\sin ^{2} \varphi \cos ^{3} \theta>\alpha_{a a c}^{R}\right. \\
& \left.+<\sin ^{2} \varphi \cos \theta+\cos ^{3} \theta \cos ^{2} \varphi>\alpha_{b b c}^{R}\right] \\
\chi_{y z y, s}^{(2)}= & \frac{N_{s}}{2}\left[<\sin ^{2} \theta \cos \theta>\alpha_{c c c}^{R}-<\sin ^{2} \theta \cos \theta \sin ^{2} \varphi>\alpha_{a \mathrm{acc}}^{\mathrm{R}}\right. \\
& \left.-<\sin ^{2} \theta \cos \theta \cos ^{2} \varphi>\alpha_{\mathrm{bbc}}^{\mathrm{R}}\right]
\end{aligned}
$$

Here angular brackets indicate an average over the angular distribution of surface water molecules, $\theta$ is the angle between the surface normal and $c$ axis, and $\varphi$ is the angle between the plane of the water molecule and the plane defined by the $c$ axis and surface normal. For a reasonable description of orientational distribution, at least three parameters need to be determined. Since our polarization dependence measurements only yield the ratio between the two susceptibilities, it is easy to understand that the amount of information that can be deduced is very limited in this case. The situation will not be

considerably improved even if we measure $\chi_{\mathrm{zzz}}^{(2)}$ from measuring the spectrum with ppp polarization combination.

\section{F. SFG of Water Surface Covered by a Monolayer of Fatty Acid $\left(\mathrm{C}_{25} \mathrm{COOH}\right)$}


Langmuir monolayers on water have been widely studied as a model system for biological membranes and for their potential applications as molecular devices. ${ }^{21}$ Very detailed information about the hydrocarbon chain in fatty acid monolayer on water have been obtained from X-ray reflectivity and grazing incident diffraction (GID) measurements. ${ }^{22}$ However, studies of the interaction between the headgroups of the monolayer and the water molecules at the interface have not been possible, although this interaction is probably the most important one in determining the structure of the monolayer. SFG is an ideal technique for studying this interaction. With powerful infrared lasers presently available, vibrational spectra of both the headgroups and the water molecules can be measured. In this session, we present our preliminary study of SFG spectra of water molecules at this interface, as a function of $\mathrm{pH}$ in the bulk water, because results can be directly compared with the quartz/water interface discussed above.

Hexacosanoic acid (>99\%) was purchased from Sigma, St. Louis, MO, and the water used was Milipore filtered. Spreading and compression of the monolayer was done on a home built Langmuir trough. The surface pressure was measured by Wilhelmy plate method using a filter paper and an analytical balance. Because the equilibrium spreading pressure (ESP) is very low for fatty acid of this length, the film can be only compressed to $\sim 3 \mathrm{mN} / \mathrm{m}$ if the pressure is to remain stable during the course of the SFG measurement (typically 2 hours). Thus, all the spectra shown were taken at this pressure. Spectra at higher pressure $(\sim 30 \mathrm{mN} / \mathrm{m})$ have also been taken by continuously monitoring the surface pressure and maintaining it with a computer controlled stepper motor. No significant difference was observed. The $\mathrm{pH}$ of the subphase was controlled by using a thoroughly 
equilibrated solution with the desired $\mathrm{pH}$ (by addition of acid $(\mathrm{HCl})$ or base $(\mathrm{NaOH})$ ) to prepare the fatty acid monolayer.

Fig. 7 shows SFG spectra of this system at different $\mathrm{pH}$ values. The acid molecule that we have chosen to study has a sufficiently long hydrocarbon chain, so that even at the highest $\mathrm{pH}$, no material loss due to dissolution is observed. Spectra of both the $\mathrm{CH}$ stretching modes from the hydrocarbon chains and the $\mathrm{OH}$ stretching modes from the surface water molecules and possibly the headgroups of the fatty acid monolayer, are taken. As the signal from the hydrocarbon chains does not vary considerably with $\mathrm{pH}$, we conclude that the density of the monolayer does not play a significant role. As can be seen in Fig. $6(\mathrm{~b})$, the spectra at $\mathrm{pH}$ higher than 7 are remarkably similar to the spectra of the quartz/water interface at $\mathrm{pH} \geq 5.6$. This suggests that at high $\mathrm{pH}$, the molecular structure and orientation of surface water molecules are largely controlled by the strong surface electric field resulting from the ionization of surface groups, in this case, the acid groups. For low $\mathrm{pH}$, however, the spectra are considerably different from the quartz/water interface, suggesting that at low $\mathrm{pH}$ the interaction of water molecules with fatty acid monolayer is mainly through hydrogen bonding. The peak at around $3050 \mathrm{~cm}^{-1}$, present in spectra at $\mathrm{pH} \leq 3.0$, is probably due to the $\mathrm{OH}$ stretch mode of fatty acid monolayer, because the respective frequency of water molecules was never found to be below 3100 $\mathrm{cm}^{-1}$. ${ }^{13(b)}$ The peak at $\sim 3600 \mathrm{~cm}^{-1}$ is most likely due to water molecules having very weak hydrogen bonding with its neighbors. Since the spectrum is so different from the spectra we have seen for other interfaces, the assignment is likewise different. Although it is difficult to get the full picture with our spectra alone, the disappearance of the peak at $3050 \mathrm{~cm}^{-1}$ for $\mathrm{pH} \geq 3.0$ seems to suggest a major reorientation of the headgroups of the 
fatty acid monolayer. For $\mathrm{pH} \leq 5.6$, the prominence of the peak at $3600 \mathrm{~cm}^{-1}$ is certainly counterintuitive if one realizes that there are a large number of hydrogen bonding agents present at this interface. More work is currently under way to clarify the water structure at this interface.

\section{G. Conclusions}

In summary, we have used infrared-visible SFG to study the OH stretch vibrations of water molecules at fused quartz/water interfaces with different bulk $\mathrm{pH}$ values. We have found that the orientation and bonding structure of the interfacial water molecules are strongly influenced by electrostatic interaction and hydrogen bonding with the surface. The outcome is highly dependent on the balance between them. Such behavior has long been postulated in electrochemistry at metal electrodes. ${ }^{1}$ More specifically, we have found that the interfacial water structure can undergo an order-disorder-order change, accompanied by a flip of the molecular orientation when the bulk $\mathrm{pH}$ is varied. Several monolayers of interfacial water molecules can be polar ordered if the surface is highly ionized at high $\mathrm{pH}$ values. For a fatty acid monolayer on water, similar behavior was also observed, especially for high enough $\mathrm{pH}$ values where the structure of surface water molecules is largely controlled by the surface field. These results are generally important to many surface phenomena involving the solvent structure of water. 


\section{References}

1. W. R. Fawcett, J. Chem. Phys. 93 (9), 6813 (1990); J. O M. Brockris and S. U. M. Khan, Quantum Electrochemistry (Plenum, New York, 1979), Chapter 1; N. F. Mott and R. J. Watts - Tobin, Electrochemica Acta, 4, 79 (1961)

2. J. N. Israelachvili, Intermolecular and Surface Forces (Academic, London,1989), Chapter 15-17.

3. R. Pethig, in Annual Review of Physical Chemistry, Vol. 43 (1992) pp. 177, edited by H. L. Strauss.

4. S.L. Carnie and G.M. Torrie, Adv. Chem. Phys., 56, 141 (1984); K.Raghavan, K. Foster and M. Berkowitz, Chem. Phys. Lett., 177 (4,5) 426 (1991); D.A. Rose and I. Benjamin, J. Chem. Phys. 98 (3) 2283 (1993), and references therein.

5. (a) R. M. Pashley and J. N. Israelachvili, J. Colloid \& Interface Sci. 101 (2), 511 (1984); (b) S. Marielja and N. Radic, Chem. Phys. Lett. 42 (1), 129 (1976).

6. R. Lenk, M. Bonzon and H. Greppin, Chem. Phys. Lett. 76 (1), 175 (1980).

7. P. Guyot-Sionnest, J. H. Hunt and Y. R. Shen, Phys. Rev.Lett., 59, 1597 (1988); R. Superfine, J. H. Huang and Y. R. Shen, Phys. Rev. Lett. 144, 1 (1991).

8. S. Ong, X. Zhao and K. B. Eisenthal, Chem. Phys. Lett., 191 (3), 327 (1992).

9. Q. Du, R. Superfine and E. Freysz and Y. R. Shen, Phys. Rev. Lett., 70 (15), 2313 (1993).

10. L. T. Zhuravlev, Langmuir, 3, 316 (1987). 
11. For a recent review, see H. E. Bergna, in The Colloid Chemistry of Silica, p.21-38, (American Chemical Society, Washington D.C., 1994).

12. R. Superfine, J. H. Huang and Y. R. Shen, Optics Letters, 15 (22), 1276 (1990).

13. (a) D. E. Hare and C. M. Sorensen, J. Chem. Phys. 96 (1), 13 (1992); (b) J. R. Scherer, in Advances in Infrared and Raman Spectroscopy, Vol. 5 pp. 149, edited R. J. H. Clark and R. E. Hester (Heyden, Philadelphia, 1978); (c) P. A. Gignere, J. Raman Spec. 15 (5), 354 (1984).

14. R. K. Iler, The Chemistry of Silica (Wiley, New York, 1979) pp.622-729.

15. G. R. Wiese, R. O. James, D. E. Yates and T. W. Healy, in Int. Rev. Sci., Phys. Chem. Ser. 2, Vol. 6 pp. 53-102, edited by J. O M. Brockris (Butterworths, London 1976).

16. M. Watanabe, A. M. Brodsky and W. P. Reinhardt, J. Phys. Chem., 95, 4593 (1991).

17. M. Gavish, J.-L. Wang, M. Eisenstein, M.Lahav and L.Leiserowitz, Science, 256, 815 (1992); P. Hobbs, Ice Physics pp.500 (Clarendon Press, Oxford, 1974).

18. R. Kjellander and S. Marcelja, Chem. Phys. Lett., 120 (4,5), 393 (1985).

19. K. Klier and A. C. Zettlemoyer, J. Colloid \& Interface Sci., 58 (2), 216 (1977).

20. In Ref. 9, we showed that $\mathrm{OH}$ groups from an insoluble alcohol monolayer can produce similar polar ordering at the water surface as for the quartz surface at low $\mathrm{pH}$.

21. G. L. Gaines, Jr., Insoluble Monolayers at Liquid-Gas Interfaces (Wiley, New York, 1966). 
22. F. Leveiller, D. Jacquemain, L. Leiserowitz, K. Kjaer, and J. Als-Nielsen, J. Phys. Chem. 96, 10380 (1992). 


\section{Figure captions}

Fig. 1: Experimental setup for measuring the phase of by interference between the SFG of sample and a nonlinear crystal. In the simple compensator made of fused silica, one wedge is translated against a fixed wedge to change the relative optical phases of the three beams.

Fig. 2: SFG spectra from the quartz/water interfaces with different $\mathrm{pH}$ values of the bulk water : a) $\mathrm{pH}=1.5$; (b) $\mathrm{pH}=3.8 ;$ (c) $\mathrm{pH}=5.6$; (d) $\mathrm{pH}=8.0$; (e) $\mathrm{pH}=12.3$. (f) $\mathrm{SFG}$ spectrum from the quartz/ice interface.

Fig. 3: SFG spectra from quartz/water interfaces with (a) $\mathrm{pH}=1.5,0.5 \mathrm{M} \mathrm{NaCl}$; (b) $\mathrm{pH}=$ $5.6,0.1 \mathrm{M} \mathrm{NaCl}$; and (c) $\mathrm{pH}=12.3,0.5 \mathrm{M} \mathrm{NaCl}$; in bulk water.

Fig. 4: SFG spectra for different polarization combinations from a quartz/water interface with the bulk water at (a) $\mathrm{pH}=1.7$; (b) $\mathrm{pH}=5.6$; (c) $\mathrm{pH}=12.3$.

Fig. 5: Comparison of the interference pattern of the $3200 \mathrm{~cm}^{-1}$ peak for the low $\mathrm{pH}$ (circles) and high pH caes (squares).

Fig. 6: SFG spectra from water surface covered by a fatty acid $\left(C_{26}\right)$ monolayer, the surface pressure is $3 \mathrm{mN} / \mathrm{m}$, the polarization of the $\mathrm{SF}$ output, visible input, and infrared input are $\mathrm{s}, \mathrm{s}$, and $\mathrm{p}$, respectively. 


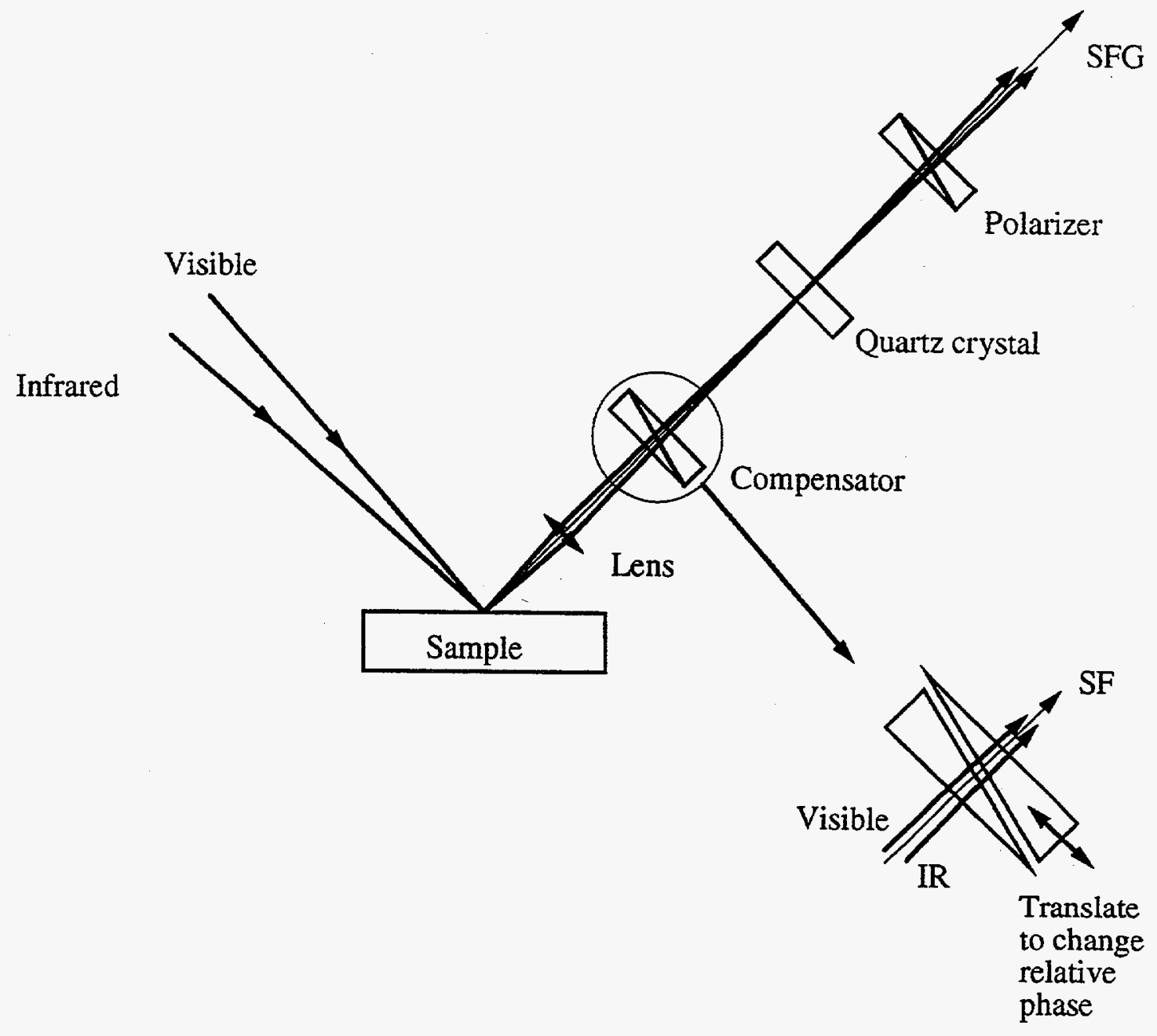

Fig. 1 


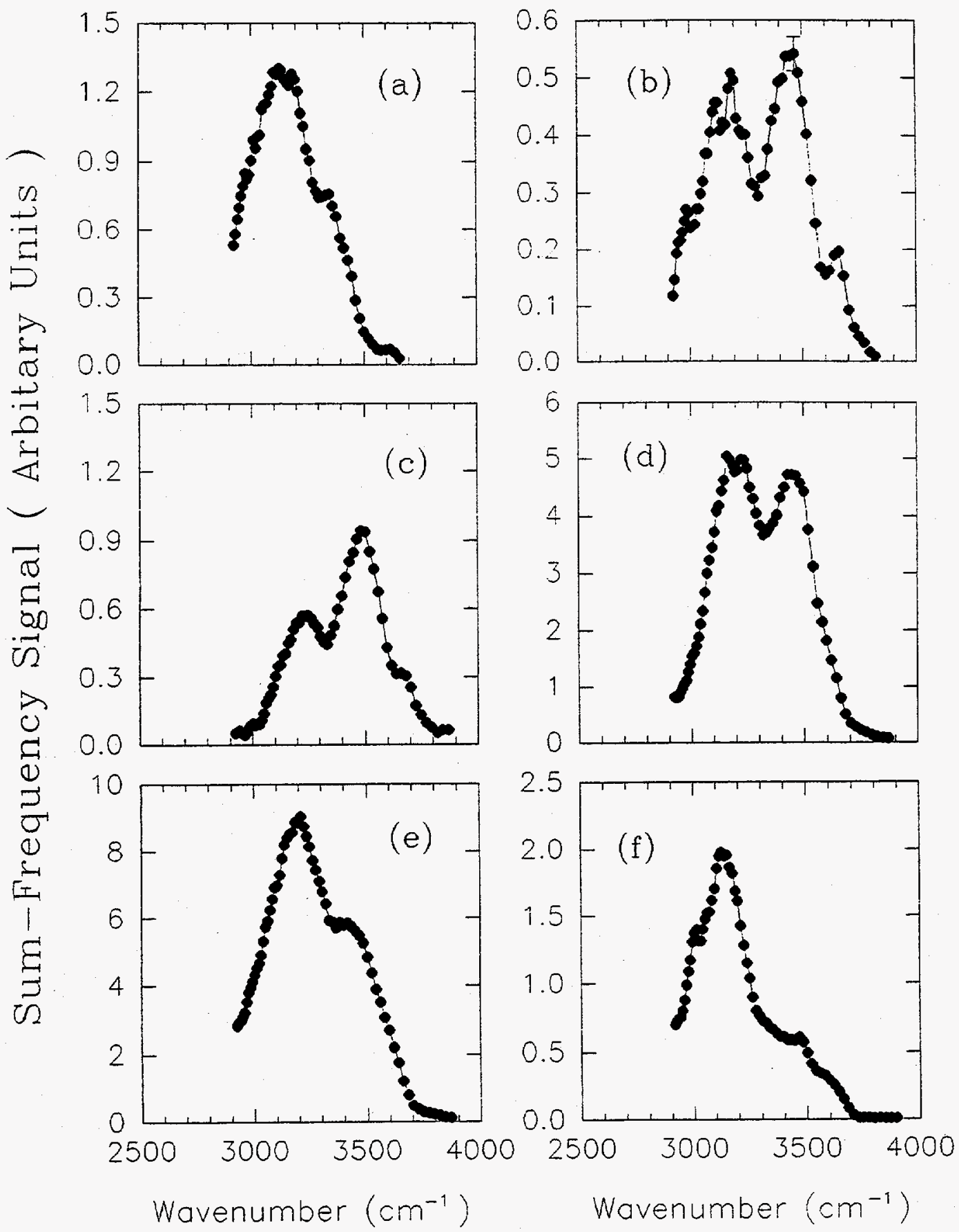

Fig. 2 


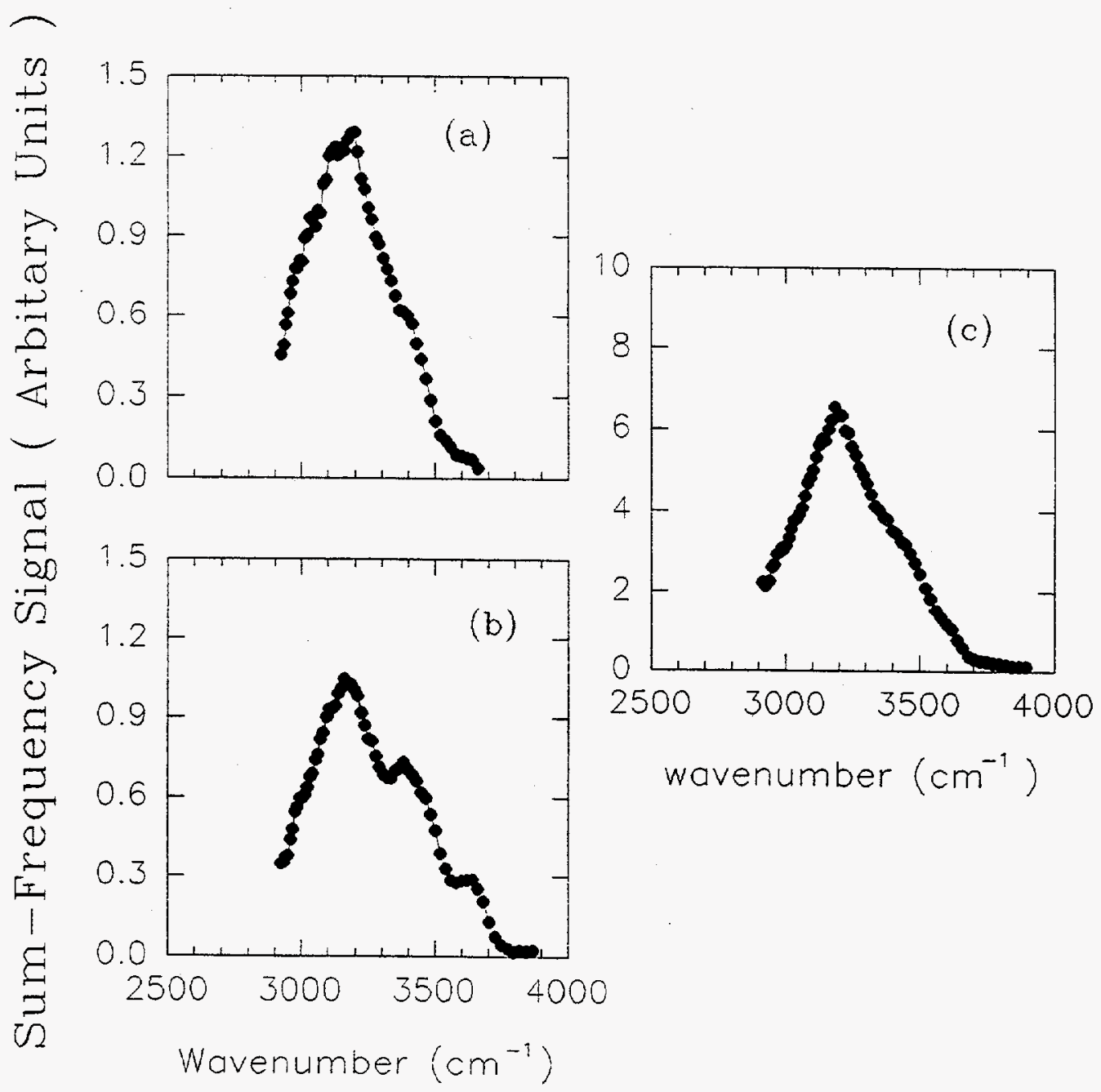

Fig. 3 


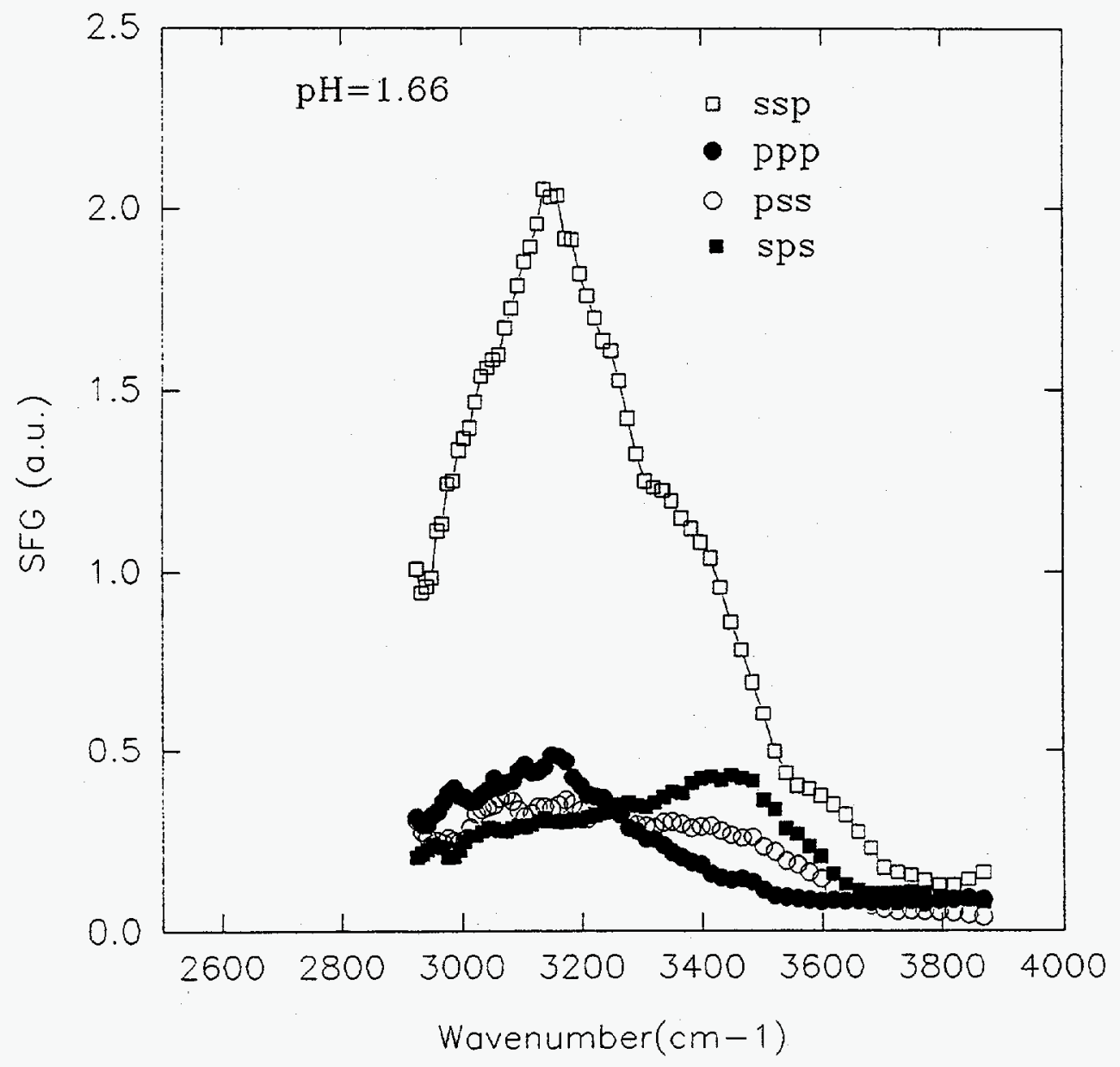

Fig. $4 a$ 


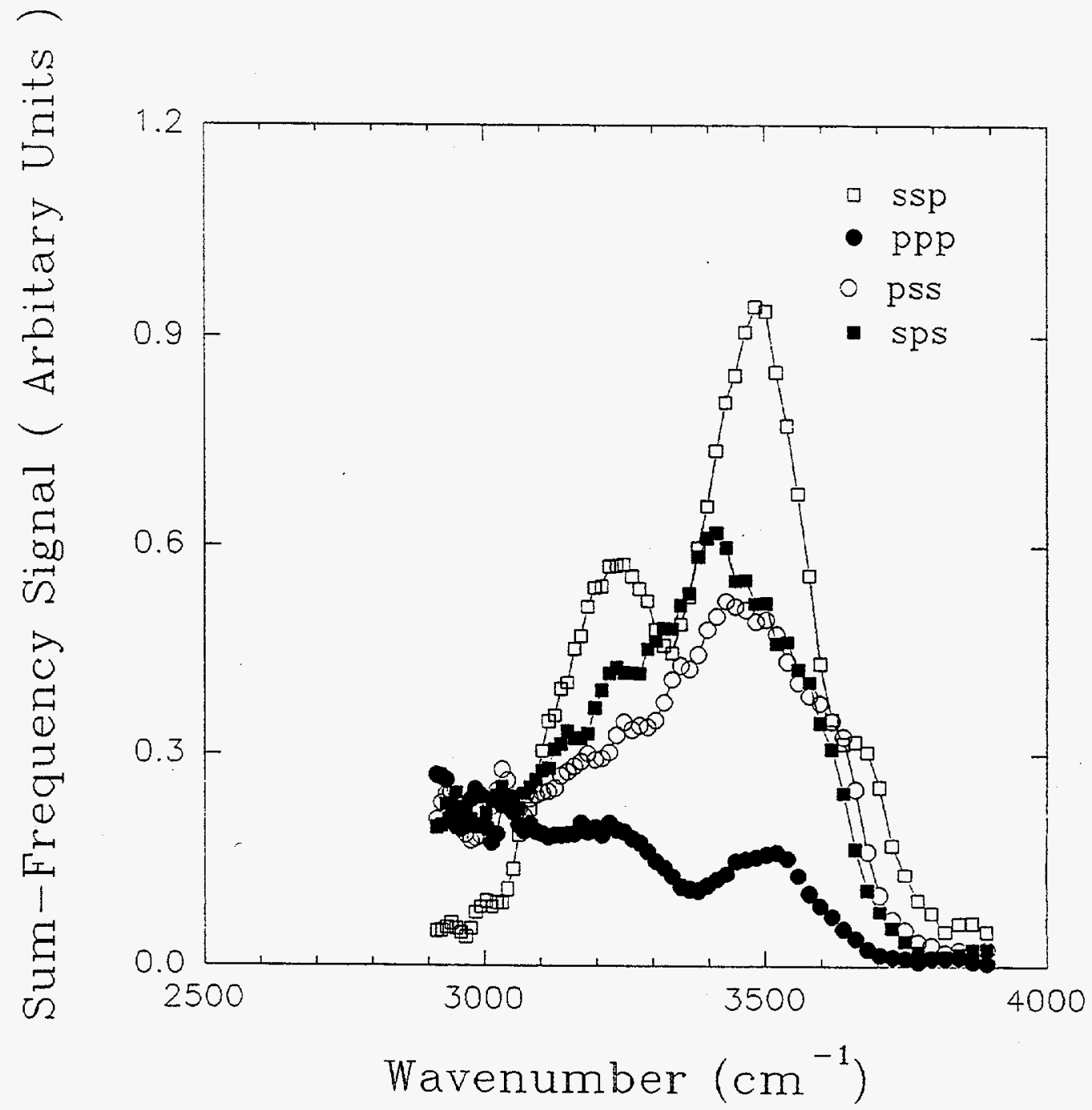

Fig. $4 \mathrm{~b}$ 


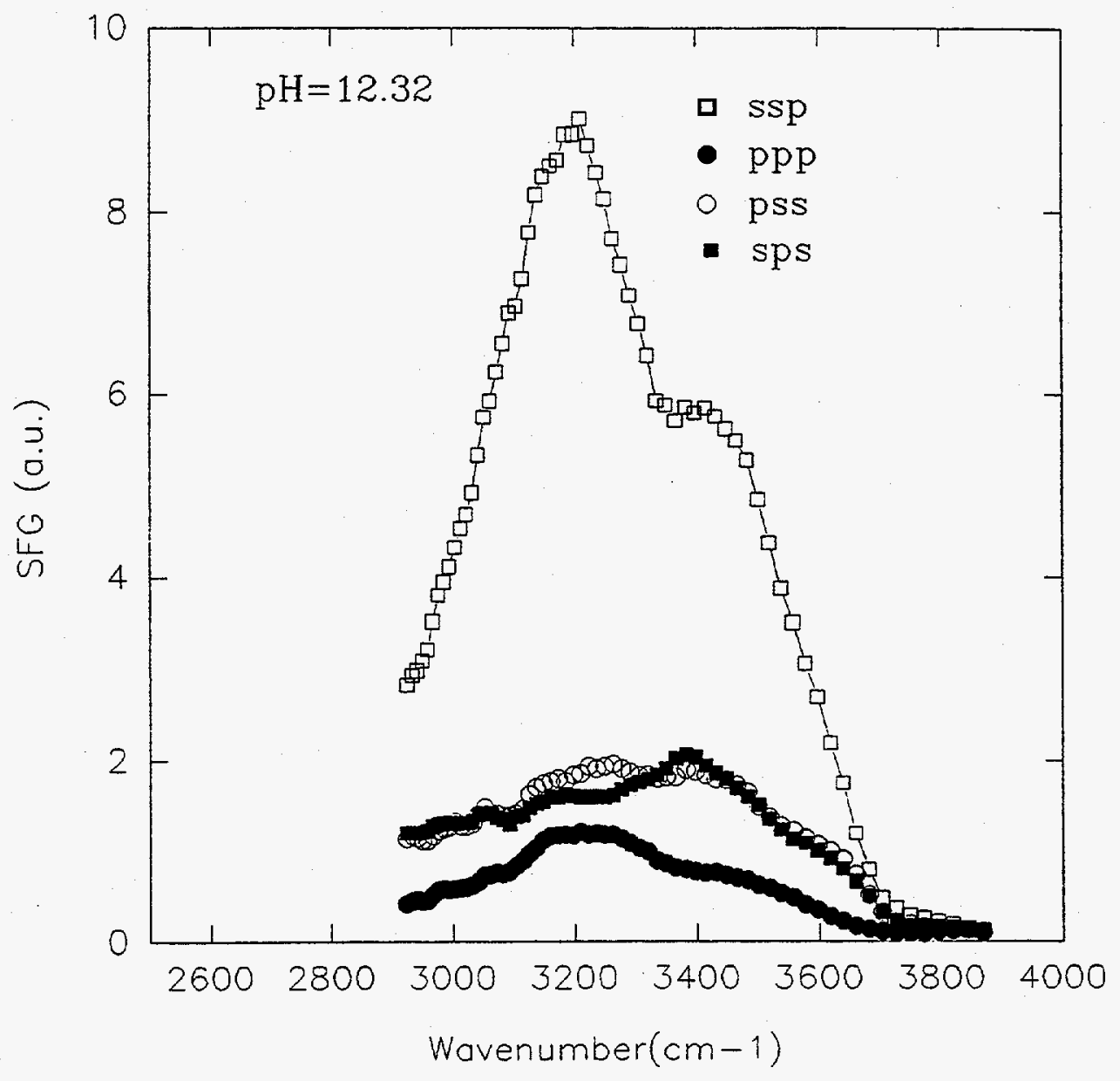

Fig. $4 c$ 


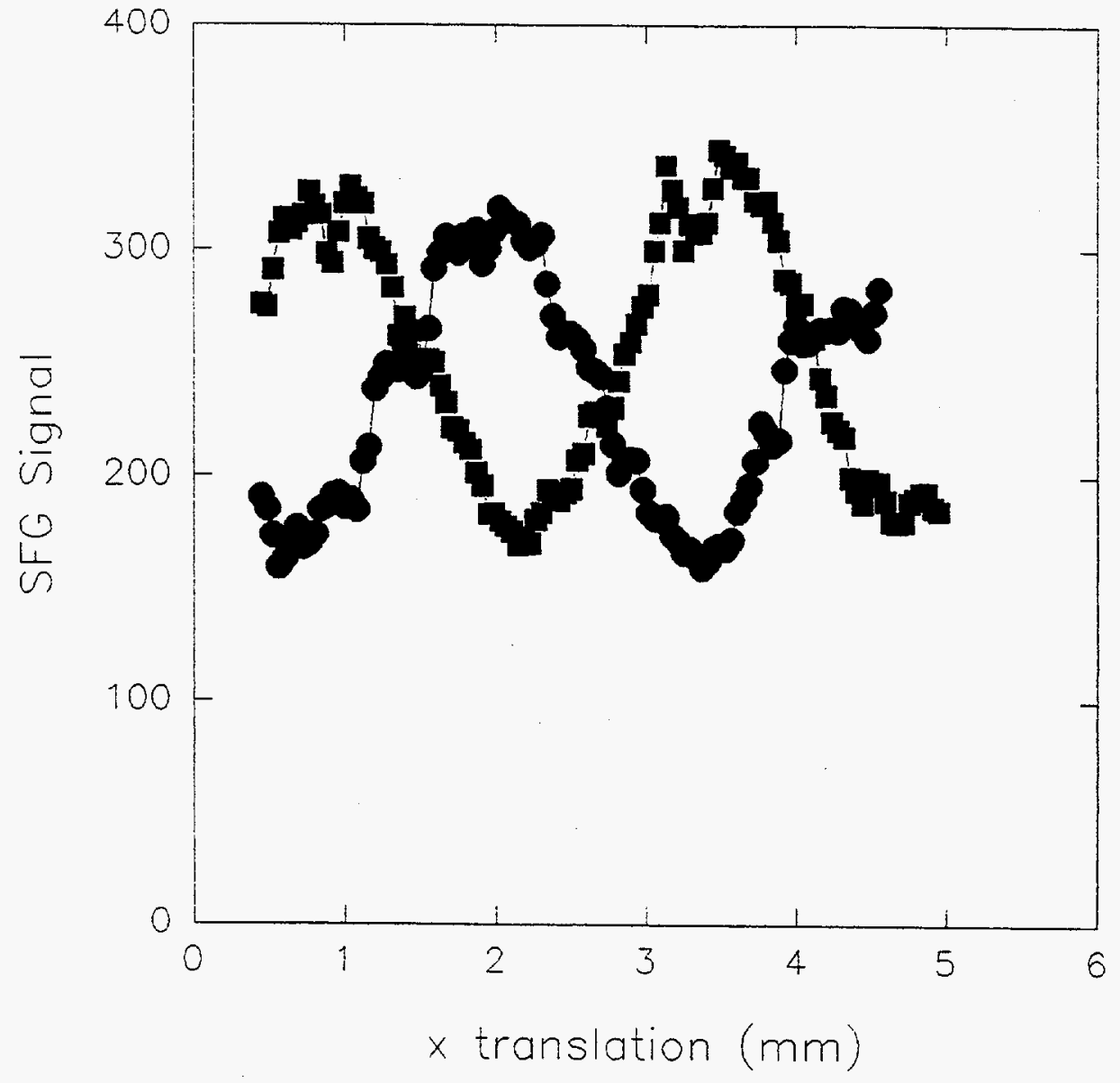

Fig. 5 


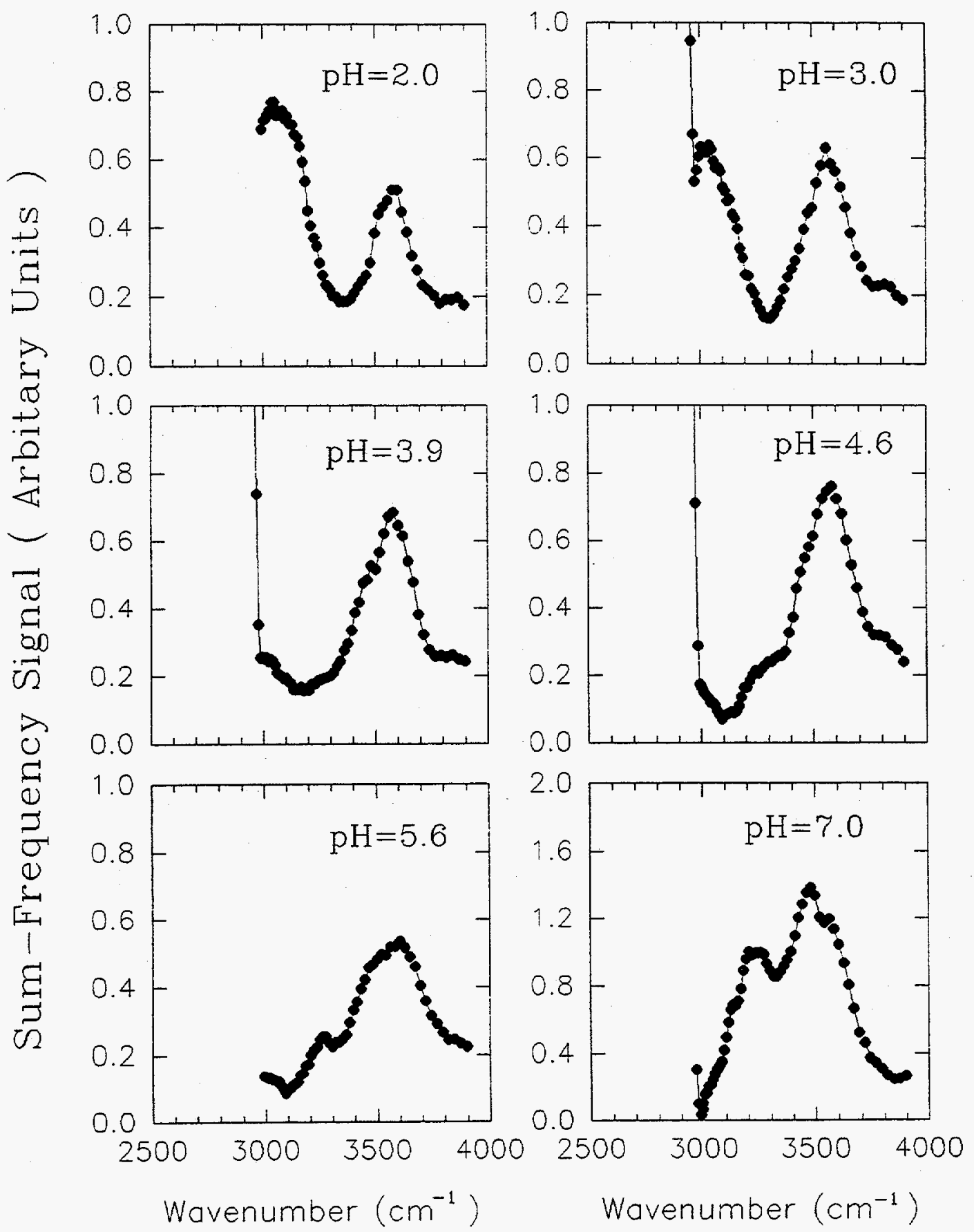

Fig. 6a 

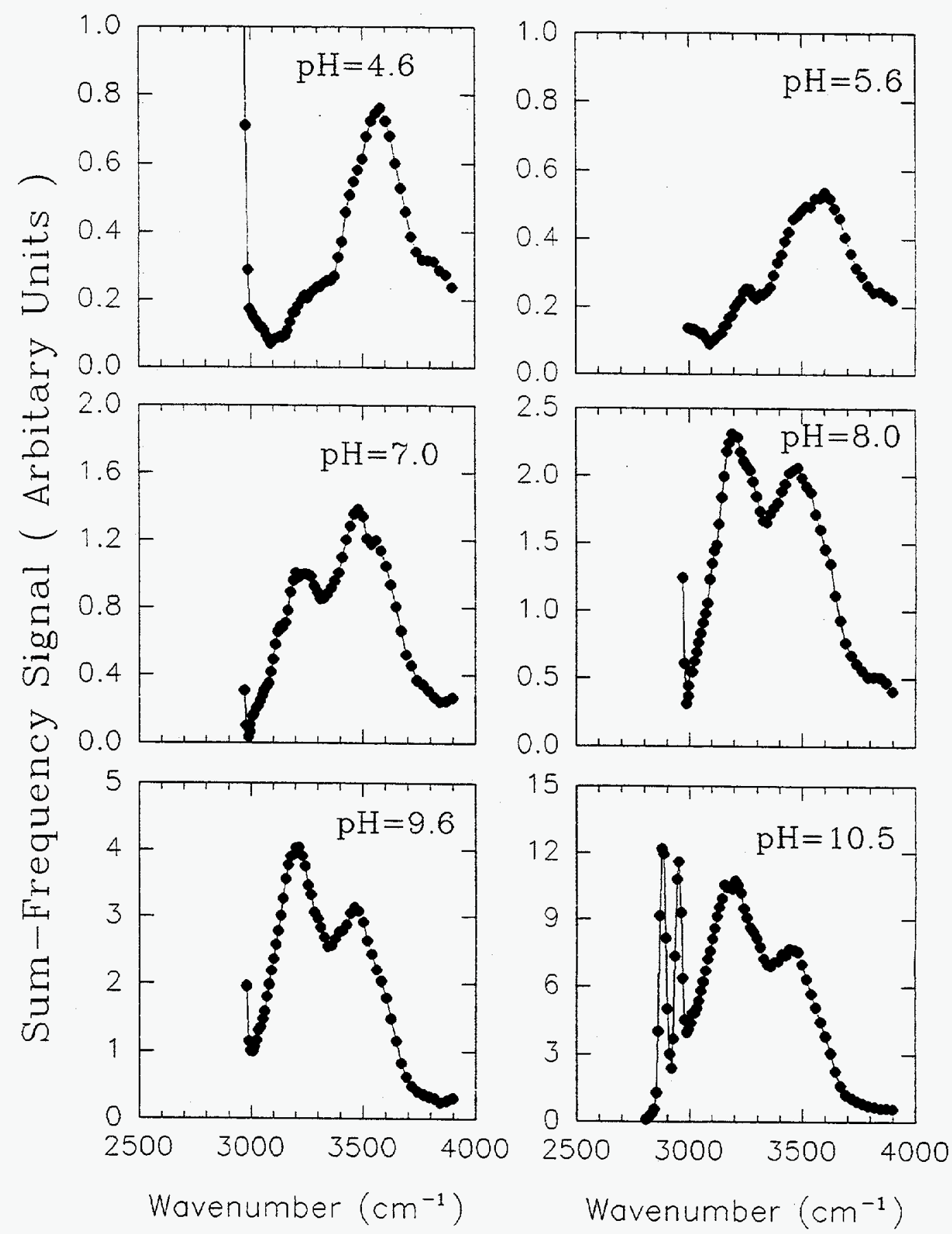

Wavenumber $\left(\mathrm{cm}^{-1}\right)$

Fig. 6b 


\section{Hydrogen Bonding and Hydrophobicity}

\section{A. Introduction}

Knowledge of interfacial water structure near hydrophobic surfaces is crucial for the understanding of many important surface problems involving water. For instance, wetting or non-wetting is a familiar phenomenon, but there is not yet a clear physical picture of the phenomenon at the molecular level. ${ }^{1}$ Hydrophobic interactions are responsible for the formation of micelles ${ }^{2}$ and play an important role in organizing constituent molecules of living matter into complex structural entities such as membranes. ${ }^{2,3}$ Understanding of hydrophobic interfaces can also provide a better picture of how large hydrophobic solute particles are surrounded by water molecules when immersed in water. ${ }^{4}$ Thus the importance of such interfaces has stimulated a number of theoretical studies with numerical calculations in the past. ${ }^{5-7}$ Experimental research on the topic has, however, been rare or nonexistent because of a lack of suitable techniques. In this chapter, we discuss the vibrational spectroscopic measurements on two hydrophobic interfaces. One is on a water/solid interface where the solid surface is rendered hydrophobic by a monolayer coating of the surfactant OTS [octadecyltrichlorosilane, $\left.\mathrm{CH}_{3}\left(\mathrm{CH}_{2}\right)_{16} \mathrm{CCl}_{3}\right]$, and the other is on a water/hexane interface. The spectra are compared with that of the water/air interface which can also be regarded as hydrophobic. In all cases, the hydrophobic interaction is characterized by the appearance of a free $\mathrm{OH}$ stretch peak of water in the spectra. The bonded $\mathrm{OH}$ spectra of water of different cases show some clear differences, suggesting a difference in their interfacial water structure. The one 
against the solid surface appears to be more bonded-ordered. We have also probed a partly wettable surface prepared by a monolayer coating of surfactants with loosely packed hydrocarbon chains. No free $\mathrm{OH}$ peak was observed in that case.

\section{B. Theory and Experiments}

The theory of SFG has been described previously. ${ }^{8-10}$ Within the electric dipole approximation, the process of SHG is forbidden in a centrosymmetric bulk medium like water, but allowed at the surface where the inversion symmetry is broken. For water interfaces, it has been shown in Ref. 9 that the quadrupolar contribution from the bulk water is not appreciable and that the SFG in reflection comes mainly from the dipolar contribution of interfacial water molecules with preferred polar orientations.

The experimental setup for our SFG measurement has also been described elsewhere. ${ }^{8,11}$ Briefly, a picosecond passive-active mode-locked Nd:YAG laser system produced $0.5 \mathrm{~mJ}$ visible pulses at $0.532 \mu \mathrm{m}$ and $0.2 \mathrm{~mJ}$ infrared pulses tunable from 2700 to $3900 \mathrm{~cm}^{-1}$. Both the visible and the infrared pulses were incident at angles of around $47^{\circ}$ from the hydrophobic side and were overlapped at the water interface to be probed. Ultra pure water (resistivity greater than $18 \mathrm{M} \Omega . \mathrm{cm}$, obtained from a Millipore filtration system) and a Teflon cell were used in the experiment. The hydrophobic solid surface was prepared by depositing a monolayer of OTS on the surface of a fused quartz window with a standard self-assembly technique. ${ }^{12}$ The hydrophobicity of the surface arises from the layer of closely packed hydrocarbon chains. The hydrophobic liquid/water interface was prepared by depositing a thin layer of hexane onto water surface. This is 
possible because hexane can wet the water surface and is lighter than water. The exact thickness of the hexane is not known, but it is on the order of microns, so for practical purposes the interface is the same as that of bulk liquid/liquid, and any absorption of $\mathbb{R}$ laser radiation due to the hexane layer can be ignored. The partly wettable surface was prepared by adsorbing onto a fused quartz substrate a monolayer of DMOAP $[\mathrm{N}, \mathrm{N}-$ dimethyl- $N$-octadel-3-aminopropyl-trimethoxysilychloride, $\mathrm{CH}_{3}\left(\mathrm{CH}_{2}\right) 17(\mathrm{Me})_{2} \mathrm{~N}^{+}$$\left.\mathrm{CH}_{2}\right)_{3} \mathrm{Si}(\mathrm{OMe})_{3} \mathrm{Cl}^{-}{ }^{13}$

\section{The SFG Spectra of Water Molecules at Hydrophobic Interfaces}

The SFG spectrum in the OH stretch region obtained from the quartz/OTS/water interface is shown in Fig. 1(A). The SF output, visible input, and IR input are s-, s-, and ppolarized respectively (denoted by ssp). The results are similar to those obtained from a vapor/water interface, ${ }^{9}$ the ssp spectrum of which is shown in Fig. 1B for comparison. In both cases, the spectrum exhibits a sharp peak at $3680 \mathrm{~cm}^{-1}$ that can be assigned to the non-hydrogen-bonded $\mathrm{OH}$ (free $\mathrm{OH}$ ) stretch vibration. ${ }^{9}$ The broad spectral features between 3000 and $3600 \mathrm{~cm}^{-1}$ are due to hydrogen-bonded $\mathrm{OH}$ stretches.

The presence of the sharp peak at $3680 \mathrm{~cm}^{-1}$ in the spectrum indicates the existence of free $\mathrm{OH}$ bonds of water at the quartz/OTS/water interface, with the $\mathrm{H}$ atom pointing toward the solid wall. A very similar spectrum is also observed for the hydrophobic liquid/water interface, as shown in Fig. 1 (C). Hydrophobicity is therefore characterized by the lack of binding interactions between the protruding $\mathrm{OH}$ bond and the surface. In this respect, the vapor/water interface is also hydrophobic. The presence of free 
$\mathrm{OH}$ bonds at a hydrophobic surface can be physically understood as follows. Water molecules prefer to be tetrahedraly bonded to four neighbors in bulk water. This arrangement is not possible for the water molecules at a hydrophobic interface, because on one side there are no neighbors that can be hydrogen bonded to, so a fraction of these hydrogen bonds must be broken. For minimization of the surface free energy, the total number of hydrogen bonds each surface water molecule possesses should still be maximized.

The broad spectral features in the region from 3000 to $3500 \mathrm{~cm}^{-1}$ are due to hydrogen bonded $\mathrm{OH}$ stretching modes. The peak at $\sim 3200 \mathrm{~cm}^{-1}$ is generally attributed to the in phase vibrations of the coupled symmetric $\mathrm{OH}$ stretch mode of the tetrahedraly coordinated water molecules (The two OHs of the water molecules are hydrogen-bonded to the neighboring molecules with equal strength) ${ }^{14}$ This peak dominates in the quartz/ice spectrum shown in Fig. 1(D) and is an indication of bond ordering (or ice-like structure) in the water molecular arrangement. The peak at $\sim 3400 \mathrm{~cm}^{-1}$ is assigned to the symmetric stretch mode of asymmetrically bonded water molecules (molecules with one $\mathrm{H}$ strongly hydrogen bonded and the other $\mathrm{H}$ weakly bonded to neighboring molecules) ${ }^{14}$ At the hydrophobic water interfaces, this peak can be assigned to the water molecules with one free $\mathrm{OH}$ and one $\mathrm{OH}$ strongly hydrogen bonded. This peak then represents bond disordering in the water molecular arrangement. As seen in Fig. 1(A), the spectrum of the water/hydrophobic solid interface in the bonded $\mathrm{OH}$ region is dominated by the peak at $\sim 3200 \mathrm{~cm}^{-1}$ with only a weak shoulder at $3400 \mathrm{~cm}^{-1}$. It closely resembles that of the quartz/ice interface depicted in Fig. 1(D), and therefore indicates that water molecules at 
this hydrophobic interface are well bond-ordered. This is actually in fair agreement with the prediction of a recent molecular dynamics simulations study. ${ }^{6}$ In that study, it was suggested that due to the need to maintain the maximum number of hydrogen bonds and the need to satisfy the packing restrictions imposed by a solid wall, the surface water molecules are arranged into an ice-like structure.

The vapor/water interface with about the same number of dangling $\mathrm{OH}$ bonds is expected to follow roughly the same model. The difference is in the rigidity of the interface. The non-rigid water/vapor interface prevents the surface water molecules from being highly bond-ordered. This is reflected in the appearance of the pronounced bond disordered peak at $3400 \mathrm{~cm}^{-1}$ in the spectrum of Fig. 1(B). The same spectra should be expected for a hydrophobic water/oil interface. Indeed, this was found to be the case with the hexane/water interface. As shown in Fig. 1(C), the SFG spectrum of the water/hexane interface is similar to that of the water/vapor interface in Fig. 1(B). A recent molecular dynamics study on the hexane/water interface did not find any preferred orientation for water molecules at the interface, but this may simply be due to fact the interface modeled in the simulation was too rough. A more recent simulation of the decane/water interface suggested that the orientation of water molecules at the interface is quite similar to that of the vapor/water interface, ${ }^{7}$ consistent with our experiment.

In chapter III we have shown that there is about $25 \%$ of surface water molecules have free $\mathrm{OH}$ bonds at the vapor/water interface. The same is likely to be true for the quartz/OTS/water interface, and is supported by nearly the same relative strength of the free $\mathrm{OH}$ peak (corrected by Fresnel factors) in the spectrum of Fig. 1(A) as compared to that of Fig. 1(B). 


\section{Interfacial Water Structure at Hydrophobic Interfaces}

For a realistic model of water structure at a hydrophobic interface, as has been mentioned above, it is very important to maximize the number of hydrogen bonds for the surface water molecules. This is a formidable task, as the hydrogen bonding of the water molecules is intrinsically three dimensional. In order to construct a model of the surface molecules, the exact bonding arrangement of the surface molecules with bulk molecules will also have to be specified, otherwise the interaction between surface layer and bulk molecules can introduce an error in the total free energy of the system of the same order of magnitude as the interaction energy between the external surface and surface water molecules. Any attempt to start with water clusters will not be successful because even if one can obtain the correct structure for water clusters (which itself is a very difficult task, considering the large number of different possibilities of hydrogen bonding), one still has to face the task of properly accounting for the interaction between the clusters. For small clusters this interaction can be as important as the interactions inside the clusters.

Fortunately, we can start with the quartz/OTS/water interface. As described above, our spectrum suggests that a similarity should exist between the structure of ice and that of the water molecules at this interface. Close to a solid wall, both experimenta ${ }^{15}$ and theoretical ${ }^{6}$ studies suggest that water molecules adopt a layered structure, with the density of the layer closest to the solid surface well above the bulk density. In Fig. 2 the structure of hexagonal ice (Ih) is shown. As we can see, along the c-axis, hexagonal ice has a layered structure with hydrogen bonding between layers. For a structure model of the hydrophobic solid/water interface, we want to find a plane in ice which has the highest 
surface density (to achieve close packing for the surface layer) and lowest number density of hydrogen bonds between planes (to reduce the surface energy). Both of these requirements can be met if we choose to cleave the ice perpendicular to the c-axis, and replace half of the bulk ice with the hydrophobic solid surface. Along the surface normal and away from the solid surface, the rigid hydrogen bonding network characteristic of ice should slowly give way to the structure of liquid water, but the essence of the interfacial water structure should be captured by the ice-like hydrogen bonding network close to the surface. As we can see, only half of the surface water molecules have broken hydrogen bonds. Of these, only half are free OHs, the rest are free oxygen lone pairs. So for this interface, $25 \%$ of surface water molecules are terminated by free $\mathrm{OHs}$, and these free $\mathrm{OHs}$ point normal to the surface. This is exactly what is found at this interface from our measurement. The better orientation of the free $\mathrm{OHs}$ at this interface results in a slightly stronger peak at $3680 \mathrm{~cm}^{-1}$ than at the vapor/water interface. According to our model, the bonded $\mathrm{OH}$ of the surface water molecules having one free $\mathrm{OH}$ should have an angle of, on average, $19^{\circ}$ away from the surface, making it very inefficient for SFG. As mentioned in chapter III, for the air/water interface, the broad distribution of this angle makes the peak at $3450 \mathrm{~cm}^{-1}$ reasonably strong. For the solid/water interface, because the distribution is much narrower, this peak is much weaker, as shown in Fig. 1 (B). In addition, our model assumes that the two $\mathrm{OH}$ bonds of a water molecule have equal probabilities of orienting along any of the four tetrahedral bonding directions, thus there is no overall dipole orientation of water molecules in the structure. This is consistent with the general conclusions from theoretical studies ${ }^{16}$ and the small surface potential measured ${ }^{17}$ at the vapor/water interface. 
Similar structures should exist at the vapor/water interface and oil/water interface, despite the difference in density profile between these interfaces and the solid/water interface. At least the average orientation for the surface water molecules and the number of broken hydrogen bonds should be the same, as indicated by the SFG spectra and discussed above. For these fluid interfaces, however, no extensive hydrogen bonding network should exist for the surface molecules as well as for the bulk molecules. Thus the orientational distribution should be much broader, giving a peak at $3450 \mathrm{~cm}^{-1}$ that is much stronger compared to the same peak at the solid/water interface.

We can compare our experimental findings at these interfaces with the results of molecular dynamics simulations studies. For the hydrophobic solid/water interface, the agreement is very good, as a similar model was proposed to explain the results from the computer simulation in ref.[6]. For the vapor/water interface, most of the molecular dynamics simulations studies seem to suggest a picture close to what we proposed in this chapter. ${ }^{18}$ Moreover, a molecular dynamics simulations study has recently been carried out to calculate the vibrational spectrum of the vapor/water interface. ${ }^{19}$ The IR spectrum calculated for this interface bears enough resemblance to our spectrum, suggesting that the agreement between theory and experiment is quite good. In that study, a strong free $\mathrm{OH}$ peak was calculated, and $15-25 \%$ of surface water molecules were found to have free $\mathrm{OH}$.

\section{E. SFG Spectrum of Water Molecules at a Partly Wettable Surface}

We have also studied the interface between water and a partly wettable solid substrate. In this case, a fused quartz surface was modified by a monolayer of DMOAP, and the advancing and receding contact angles of it were 95 and 60 degrees respectively. 
The head group of DMOAP is much larger than the cross section of the hydrocarbon chains so that our quartz surface was covered with a layer of loosely packed hydrocarbon chains with a high density of kink defects. ${ }^{8,20}$ The SFG spectra for the air/DMOAP/quartz interface is presented in Fig. 3A. The sharp peak at $2850 \mathrm{~cm}^{-1}$ in the spectrum can be attributed to the $\mathrm{CH}_{2}$ symmetric stretch mode which becomes observable when a sufficient number of kink defects appear on the alkyl chains to break the symmetry. The peak at $2940 \mathrm{~cm}^{-1}$ is mainly from the $\mathrm{CH}_{2}$ asymmetric stretch mode with perhaps some contribution from the Fermi resonance peak of the $\mathrm{CH}_{3}$ stretch. The broad but relatively weak spectral features from 3000 to $3600 \mathrm{~cm}^{-1}$ are presumably due to water molecules absorbed from air onto the DMOAP covered surface.

The SFG spectrum for the quartz/DMOAP/water interface shown in Fig. $2 \mathrm{~B}$ is very different. The reason for the disappearance of the $\mathrm{CH}_{2}$ peaks is not clear. One possibility might be that the alkyl chains have been straightened considerably by interacting with surrounding water molecules, so that the defect density is considerably reduced. However, the fact there is no peak from the $\mathrm{CH}_{3}$ groups indicate the hydrocarbon chains are still disordered to some extent, so that the end groups do not have very good orientation. Another possible explanation is that because the hydrocarbon chains are surrounded by water molecules, the spectral peaks are significantly broadened. Instead of narrow peaks, we might see a broad shoulder in this frequency range. Despite their differences, both explanations suggest that water molecules penetrate the hydrophobic barrier imposed by the hydrocarbon chains to solvate the head groups of the DMOAP monolayer. The enhanced spectral intensity of the hydrogen-bonded $\mathrm{OH}$ stretching modes results from the field orientation of surface water molecules: the ionized head groups of 
DMOAP adsorbed on the surface establish a strong surface field, much as in the case of the quartz/water interface at high $\mathrm{pH},{ }^{10}$ which can orient several layers of water molecules at the interface to yield the enhanced spectrum. No free $\mathrm{OH}$ peak is discernible in the spectrum. These observations indicate that water molecules must have surrounded the hydrophobic chains. Thus the partly wettable surface corresponds to a situation where the bulk water finds the surface covered partly by water (hydrophilic) and partly by hydrocarbons (hydrophobic).

\section{F. Conclusions}

A very detailed understanding of the molecular structure of hydrophobic water interfaces is established. The surface water molecules do not interact strongly with the opposing surface. They form a hydrogen bonding network with dangling $\mathrm{OH}$ bonds on one forth of them. This large number of dangling $\mathrm{OH}$ bonds gives rise to a large interfacial surface energy. In this respect, air or vapor (or gas) is hydrophobic and also forms a hydrophobic interface with water. The ordering of the hydrogen-bonding network of the surface layer depends strongly on the opposing hydrophobic surface. For a solid surface, the packing restriction of surface water molecules against the rigid wall forces the molecular arrangement into a more ordered ice-like structure. For vapor/water and oil/water interfaces, no such packing restriction exists and the interface water layer is less ordered. In the case of a partly wettable substrate prepared by covering the surface with a monolayer of loosely packed alkyl chains, water molecules appear to penetrate into the surfactant layer and induce a straightening of the alkyl chains. 


\section{References}

1. W. Zisman, Adv. Chem. Ser. 43, 1-51 (1964); G. M. Whitesides and P. Z. Laibinis, Langmuir 6, 87 (1990).

2. J. N. Israelachvili, Intermolecular and Surface Forces (Academic, London,1989), chap. $15-17$.

3. R. Pethig, Annu. Rev. Phys. Chem., 43, 177 (1992); C. Tanford, The Hydrophobic Effect 1-35 (Wiley, New York, ed. 2, 1980).

4. J. N. Israelachvili, Intermolecular and Surface Forces (Academic Press, London, 1989), p 102; L. P. Pratt and D. Chandler, J. Chem. Phys. 67, 3683 (1977).

5. S. L. Carnie and G. M. Torrie, Adv. Chem. Phys. 56, 141 (1984); K. Raghavan, K. Foster, M. Berkowitz, Chem. Phys. Lett. 177 (4,5), 426 (1991); D. A. Rose and I. Benjamin, J. Chem. Phys. 98 (3), 2283 (1993), and references therein.

6. C. Y. Lee, J. A. McCammion, R. J. Rossby, J. Chem. Phys. 80, 4448 (1984).

7. I. L. Carpenter and W. J. Hehre, J. Phys. Chem. 94, 531 (1990); A. R. Van Buuren, S.J. Marrink, and H. J. C. Berendsen, J. Phys. Chem. 97, 9206 (1993).

8. P. Guyot-Sionnest, J. H. Hunt, Y. R. Shen, Phys. Rev. Lett. 59, 1597 (1988); R. Superfine, J. H. Huang, Y. R. Shen, Phys. Rev. Lett. 66, 8 (1991) .

9. Q. Du, R. Superfine, E. Freysz, Y. R. Shen, Phys. Rev. Lett. 70, 2313 (1993).

10. Q. Du, E. Freysz, Y. R. Shen, Phys. Rev. Lett., 72, 238 (1994).

11. M. A. Floriano and C. A. Angell, J. Phys. Chem. 94, 4199 (1990). 
12. J. Sagiv, J. Am. Chem. Soc. 102, 92 (1980).

13. F. J. Kahn, Appl. Phys. Lett. 22, 386 (1973).

14. J. R. Scherer, in Advances in Infrared and Raman Spectroscopy, R. J. H. Clark and R. E. Hester, Ed. (Heyden, Philadelphia, 1978), Vol. 5, pp. 149-216.

15. For a recent review see, J. N. Israelachvili, Surf. Sci. Rep. 14, 109 (1992).

16. F. H. Stillinger, Jr. And A. Ben Naim, J. Chem. Phys. 47, 4431 (1967).

17. J. E. B. Randles, Phys. Chem. Liq. 7, 107 (1977); A. Borazio, J. R. Farrell and P. McTigue, J. Electroanal. Chem. 193, 103 (1985).

18. For a recent review, see A. Pohorille and M. Wilson, J. Mol. Struc. 284, 271 (1993).

19. I. Benjamin, preprint.

20. J. Y. Huang, R. Superfine, Y. R. Shen, Phys. Rev. A 42, 3660 (1990). 


\section{Figure captions}

Fig. 1: SFG spectra from (A) the quartz/OTS/water interface; (B) the air/water interface; (C) the hexane/water interface; and (D) the quartz/ice interface. The SF output, visible input and $\mathbb{R}$ input are s-, s-, and p-polarized, respectively.

Fig. 2: Crystalline structure of ice $\mathrm{Ih}$.

Fig. 3: SFG spectra from (A) the air/DMOAP/quartz interface and (B) the quartz/DMOAP/water interface. The polarization combination is the same as that of Fig. 1. 

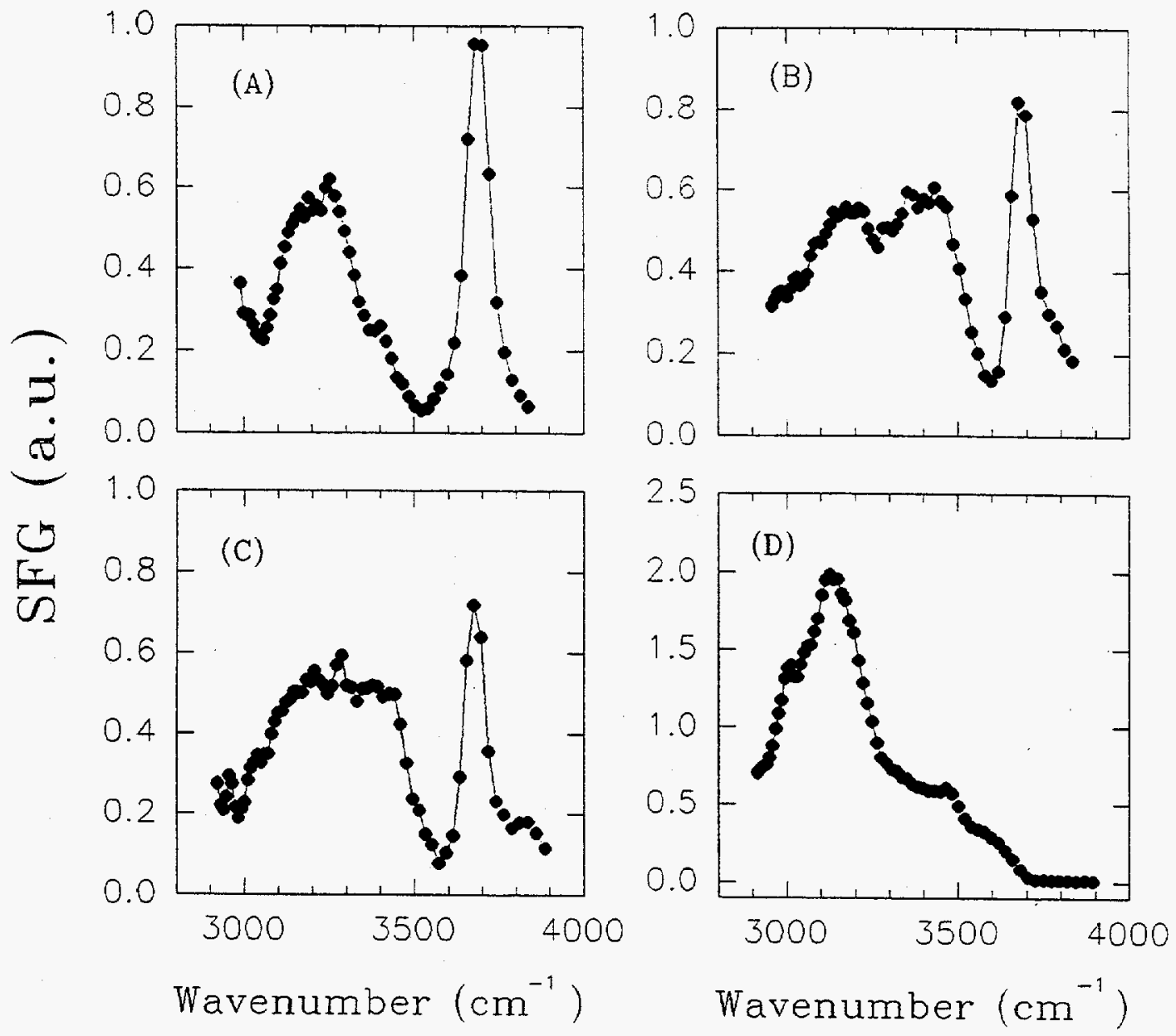

Fig. 1 


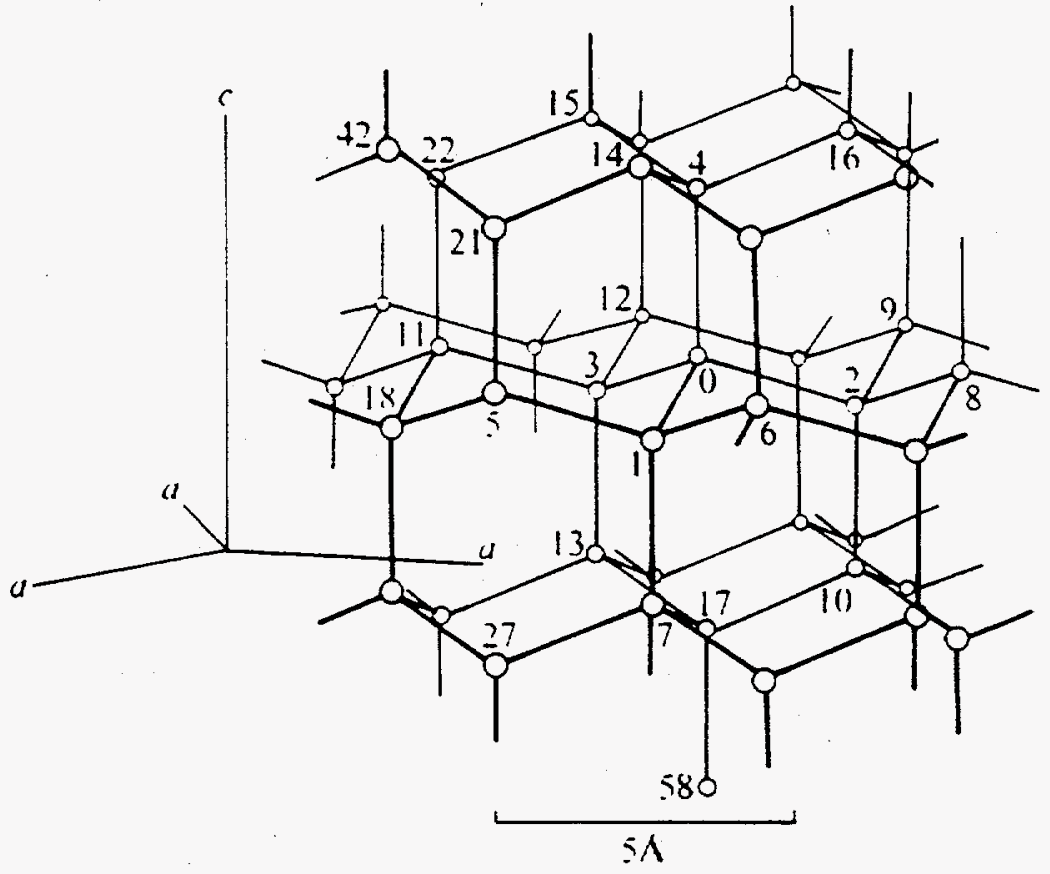

Fig. 2 


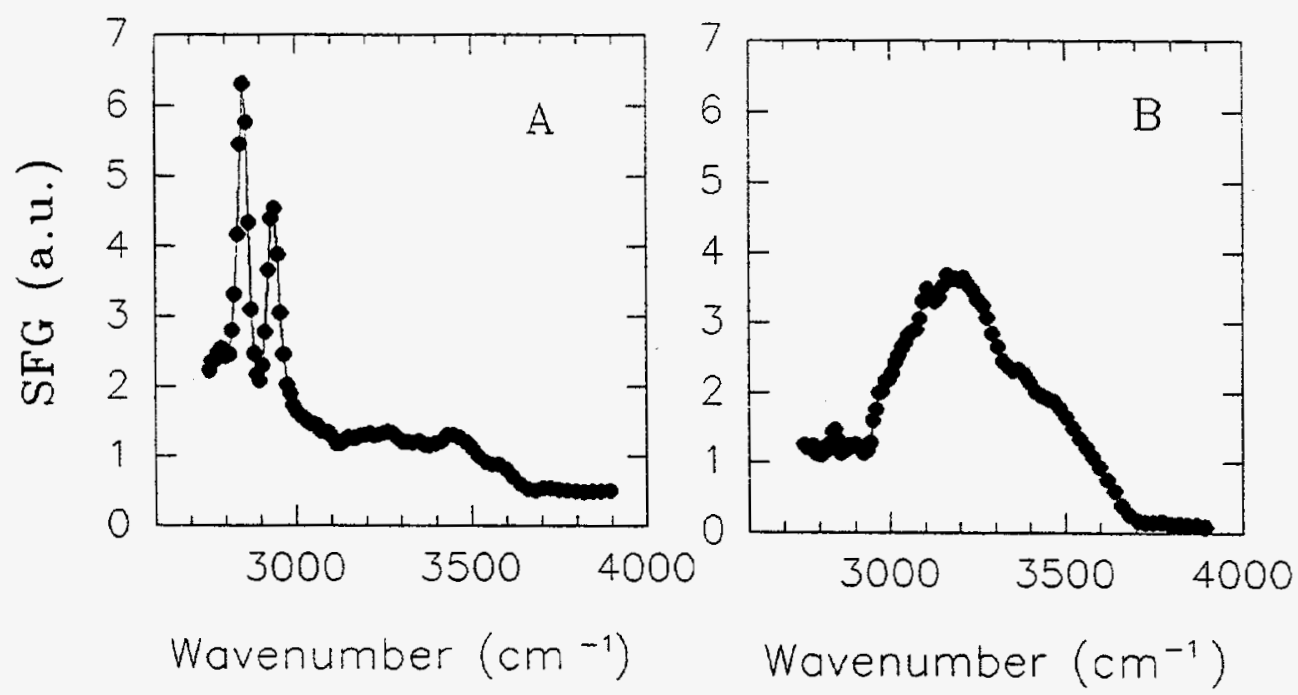

Fig. 3 


\section{Disordering of Monomolecular Films Under Pressure}

\section{A. Introduction}

Self-organized ultra-thin organic films, such as Langmuir-Blodgett films or solution self-assembled monolayers (SAM's), are attractive systems for fundamental and practical studies of lubrication. While their thickness is only a few nanometers, these films can dramatically alter the tribological properties of the underlying substrate. ${ }^{1}$ Because the development of high performance lubricants calls for a detailed understanding of friction and wear at the molecular level, ${ }^{2,3}$ these ultra-thin films are ideal model systems for study. They have been characterized using a variety of techniques, including diffraction methods, ${ }^{4}$ IR and Raman spectroscopy, ${ }^{5}$ AFM and STM, ${ }^{6}$ and nonlinear optics. ${ }^{7}$

Structural studies by various diffraction and nonlinear optical methods have been performed only under zero pressure conditions. Although monolayer structures under pressure and shear have been studied using $\mathrm{AFM}^{8-10}$ and surface force apparatus (SFA), ${ }^{11}$ the molecular conformation and orientation within the monolayers under conditions that are closer to realistic lubricating conditions, i.e., under pressure, is largely outside of their capability. Spectroscopic methods are required to derive such information. Currently, spectroscopic methods, such as infrared and Raman spectroscopy, can readily fingerprint particular chemical functionalities subjected to controlled wear. ${ }^{12,13}$ However, these methods cannot be used to monitor ultra-thin lubricant films in situ, with an applied pressure or shear, due to their limited sensitivities. 
It has recently been demonstrated that second harmonic generation (SHG) and sum-frequency generation (SFG) are ideal tools to study buried interfaces. ${ }^{14,15}$ These techniques can be readily adopted to study molecular films under static pressure to provide molecular orientation or conformation information. In this chapter, we discuss results obtained using these nonlinear optical techniques to study orientation, conformation and molecular vibrations of a variety of Langmuir-Blodgett and SAM films under applied pressure. The results indicate that the previously oriented molecules lose their polar ordering under high pressure, but recover their original orientation as soon as the pressure is released.

We complete this introduction with a brief review of some of the theoretical aspects of the compression of surfaces in the elastic regime and of the basic principles of non-linear optics as applied to the present study.

\section{A.1 Elastic Contact Between Surfaces}

The elastic deformation and stress distribution in the contact area between a flat and a sphere can be described by the standard Hertzian theory. ${ }^{16}$ The contact radius a can be determined from the following expression:

$$
a=\left(\frac{3 F R}{4 E}\right)^{\frac{1}{3}}
$$

where $\mathbf{F}$ is the total force exerted on the surface, $\mathbf{R}$ is the radius of curvature of the sphere, $\mathbf{E}$ is the reduced Young's modulus of the substrates given by $1 / E=\left(1-\sigma_{1}^{2}\right) / E_{1}+(1-$ 
$\left.\sigma_{2}^{2}\right) / E_{2}$, with $\sigma_{n}$ the corresponding Poisson ratios of the sphere and flat. The pressure $\mathbf{P}$ at position $\mathbf{r}$ inside the contact area can then be expressed as:

$$
P(r)=P(0) \sqrt{a^{2}-r^{2}}
$$

and the average pressure is

$$
\overline{\mathrm{P}}=\frac{\mathrm{F}}{\pi \mathrm{a}^{2}}=\frac{1}{\pi}\left(\frac{16 \mathrm{FE}^{2}}{9 \mathrm{R}^{2}}\right) \frac{1}{3}
$$

The pressure is only dependent on the $1 / 3$ power of the force. In order to vary the pressure by a factor of 10 , for example, the force has to be changed by a factor of 1000 . A more efficient way of varying $\mathbf{P}$ is by changing the radius of curvature $\mathbf{R}$. Combining (6.1) and (6.2), we have:

$$
\mathrm{P}=\frac{4 \mathrm{Ea}}{3 \pi \mathrm{R}}
$$

Here $\mathrm{E}=35 \mathrm{GPa}^{17}$ when both substrates are fused silica, as in present experiments. The contact radius a is readily measured with a microscope and, since $\mathrm{R}$ is known, we can easily calculate the average pressure. A typical example is $a=0.5 \mathrm{~mm}$ and $\mathrm{R}=15 \mathrm{~cm}$, yielding $P=50 \mathrm{MPa}$.

\section{A.2 Nonlinear Optics}

SHG or SFG as a probe to study monomolecular films has been described in previous publications. ${ }^{18.19}$ Within the electric dipole approximation, the process is 
forbidden in a centrosymmetric medium, but allowed at the surface where the inversion symmetry is broken. In our case, the amorphous fused quartz substrate does not contribute significantly to the signal; therefore, the second order non-linear optical response of the system is essentially generated only from the monolayer under study. The SHG and SFG signal is then proportional to the square of the effective surface nonlinear polarization:

$\mathrm{P}_{\mathrm{s}}^{(2)}\left(\omega_{3}\right)=\chi_{\mathrm{s}}^{(2)}\left(\omega_{3}=\omega_{1}+\omega_{2}\right): \mathrm{E}\left(\omega_{1}\right) \mathrm{E}\left(\omega_{2}\right)$

and can be further written as,

$S \propto\left[L\left(\omega_{3}\right) \cdot \chi_{s}^{(2)}\left(\omega_{3}=\omega_{1}+\omega_{2}\right): L\left(\omega_{1}\right) L\left(\omega_{2}\right)\right]^{2} \mathrm{I}\left(\omega_{1}\right) \mathrm{I}\left(\omega_{2}\right)$

with $\omega_{1}=\omega_{2}$ for SHG. $E\left(\omega_{\mathrm{i}}\right)$ is the electric field at the monolayer and $I\left(\omega_{\mathrm{i}}\right)$ the corresponding light intensity at the respective frequencies. $\mathrm{L}\left(\omega_{\mathrm{i}}\right)$ is the local field tensor defined as the ratio of the local field at the monolayer to the incident field. The surface susceptibility $\chi_{s}^{(2)}$ can be related to the molecular polarizability $\alpha^{(2)}$ by a coordinate transformation averaged over the molecular orientational distribution, denoted by the angular brackets:

$\chi_{s, j \mathrm{jk}}^{(2)}=\mathrm{N}_{\mathrm{s}} \sum_{\mathrm{l}, \mathrm{m}, \mathrm{n}}<(\hat{\mathrm{i}} \cdot \hat{\mathrm{l}})(\hat{\mathrm{j}} \cdot \hat{\mathrm{m}})(\hat{\mathrm{k}} \cdot \hat{\mathrm{n}})>\alpha_{\mathrm{imn}}^{(2)}$

where $\mathrm{N}_{\mathrm{s}}$ is the number of molecules per unit area, ijk the unit vectors for the laboratory frame and $\operatorname{lmn}$ for the molecular reference frame. With information on $\mathrm{N}_{\mathrm{S}}$ and $\alpha_{\operatorname{lm}}^{(2)}$ available, the measured $\chi_{s}^{(2)}$ can be directly related to the molecular orientation. In the 
case of sum-frequency generation (infrared + visible), the nonlinear polarizability near vibrational resonances can be written as the sum of a resonant and non-resonant part:

$\alpha^{(2)}=\alpha^{(2) R}+\alpha^{(2) N R}$

with

$\alpha^{(2) R}=\sum_{q} \frac{A_{q}}{\omega_{q}-\omega_{I R}-i \Gamma_{q}}$

Here $A_{q}, w_{q}$ and $\Gamma_{q}$ are respectively the strength, frequency and damping constants of the qth vibrational mode of the molecules. For the organic films with azimuthal symmetry studied in this paper, the nonvanishing elements are $\chi_{\mathrm{zz}}^{(2)}, \chi_{\mathrm{zii}}^{(2)}, \chi_{\mathrm{izi}}^{(2)}$, and $\chi_{\mathrm{iiz}}^{(2)}(\mathrm{i}=\mathrm{x}, \mathrm{y})$. In the case of $8 \mathrm{CB}$ used in our SHG study, only one dominant polarizability element $\alpha_{\xi \xi \xi}^{(2)}$ needs to be considered; ${ }^{20}$ therefore, the effective surface susceptibility elements $\chi_{s, \mathrm{jjk}}^{(2)}$ are:

$\chi_{2 z 2}^{(2)}=N_{s} \alpha_{\xi \xi \xi}^{(2)}<\cos ^{3} \theta>$

$\chi_{\mathrm{zi}}^{(2)}=\chi_{\mathrm{izi}}^{(2)}=\chi_{\mathrm{iiz}}^{(2)}=\frac{1}{2} \mathrm{~N}_{\mathrm{s}} \alpha_{\xi \xi \xi}^{(2)}<\cos \theta \sin ^{2} \theta>$

where $\mathrm{i}=\mathrm{x}$ or $\mathrm{y}, \mathrm{q}=$ the polar angle between the direction of the molecular head axis and the surface normal, $\mathrm{z}$ is the surface normal, and $\mathrm{x}$ and $\mathrm{y}$ are the coordinates in the surface plane.

For $\mathrm{CH}_{3}$, we assume a $\mathrm{C}_{3 \mathrm{v}}$ symmetry and a second order polarizability of each $\mathrm{CH}$ bond characterized by a single component $\alpha_{\xi \xi \xi}^{(2)}$ along the bond. ${ }^{21}$ Therefore, the yyz component of the second-order non-linear susceptibility can be expressed as: 
$\chi_{s, y y z}^{(2) R}=N_{s} \alpha_{\xi \xi 5}^{(2)}\left(0.275<\cos \theta>+0.165<\cos ^{3} \theta>\right)$

where $\theta$ is the angle between the symmetry axis of the $\mathrm{CH}_{3}$ and the surface normal z.

With the above, the SHG signal is further given by:

$\mathrm{S}\left(\mathrm{s}_{\mathrm{out}}, \mathrm{m}_{\mathrm{in}}\right) \propto\left[\mathrm{L}_{\mathrm{yy}}(2 \omega) \chi_{\mathrm{yyz}}^{(2)} \mathrm{L}_{\mathrm{yy}}(\omega) \mathrm{L}_{\mathrm{zz}}(\omega)\right]^{2}$

for mixed polarization input $\left(45^{\circ}\right.$ between $s$ and $\left.p\right)$ and $s$ polarization output, and

$\mathrm{S}\left(\mathrm{p}_{\text {out }}, \mathrm{m}_{\mathrm{in}}\right) \propto\left[\frac{1}{2} \mathrm{~L}_{\mathrm{zz}}(2 \omega) \chi_{\mathrm{zz}}^{(2)} \mathrm{L}_{z z}^{2}(\omega) \sin ^{3} \alpha+\frac{1}{2} \mathrm{~L}_{\mathrm{zz}}(2 \omega) \chi_{\mathrm{zx}}^{(2)} \mathrm{L}_{\mathrm{xx}}^{2}(\omega) \sin \alpha \cos ^{2} \alpha\right.$

$\left.+\frac{1}{2} L_{z z}(2 \omega) \chi_{z y y}^{(2)} L_{y y}^{2}(\omega) \sin \alpha-L_{x x}(2 \omega) \chi_{x z x}^{(2)} L_{z z}(\omega) L_{x x}(\omega) \sin \alpha \cos ^{2} \alpha\right]^{2}$

for mixed polarization input and $p$ polarization output. Here $\alpha$ is the incident angle of the laser beam. For our geometry, with a monolayer confined between two solid substrates, it has been proven by Feller $e t a l^{22}$ that $L_{\mathrm{ii}}(\omega)$ can be calculated by considering the multiple interference of the field between the two substrates, which can be expressed in terms of ordinary Fresnel factors. For example, if we scan the sample across the contact area boundary, the distance between the two surfaces varies and the local field factor $L_{\mathrm{ii}}(\omega)$ is then an oscillating function of the separation. For SFG, the signal can be similarly expressed.

\section{B. Experimental}

\section{B.1 Optical Setup}

The frequency-doubled output of a Q-switched mode-locked Nd:YAG laser was used for SHG studies. The laser beam was focused to a spot of $\sim 100 \mu \mathrm{m}$ in diameter at 
the sample surface. The SHG signal at $266 \mathrm{~nm}$ from the monolayer was detected by a photomultiplier tube. The typical laser power used was $\sim 50 \mathrm{~mJ} /$ pulse with a pulse duration of $10 \mathrm{~ns}$.

The experimental setup for SFG has been described elsewhere. ${ }^{19}$ Briefly, the laser system produced a $0.5 \mathrm{~mJ}$ visible pulse at $532 \mathrm{~nm}($ diameter $=500 \mu \mathrm{m})$ and $0.2 \mathrm{~mJ}$ tunable infrared pulses (diameter $=300 \mu \mathrm{m}$ ) from $2700 \mathrm{~cm}^{-1}$ to $3900 \mathrm{~cm}^{-1}$. The bandwidth of the infrared beam varied from about $8 \mathrm{~cm}^{-1}$ at $2900 \mathrm{~cm}^{-1}$ to about $45 \mathrm{~cm}^{-1}$ at $3700 \mathrm{~cm}^{-1}$. The infrared and visible beams were incident at an angle of about $47^{\circ}$ from the side of a fused quartz flat window and overlapped at the quartz/monolayer interface. All SFG spectra were normalized to the SFG from a quartz crystal to offset the effects of laser fluctuation and changes in the overlap due to the tuning of the infrared laser. Absorption of IR light $(\sim 10 \%)$ due to the fused quartz window was also normalized out. The polarizations for the sum-frequency output, visible input, and infrared input were $s, s$, and $p$, respectively.

An optical system was also designed and constructed for fluorescence studies of a compressed monolayer of molecules with Rhodamine $6 \mathrm{G}$ attached as the end group. The beam from an argon laser was directed through a $400 \mathrm{~mm}$ optical fiber, and focused to a spot of $300 \mathrm{~mm}$ diameter on the contacting surfaces of the quartz substrates. Fluorescent emission from this spot was collected by a lens, filtered to pass a $10 \mathrm{~nm}$ fluorescence band of Rhodamine $6 \mathrm{G}$ at $600 \mathrm{~nm}$ wavelength, and detected by a photomultiplier. The excitation beam and detection optics, mounted off-axis to avoid glare, were oriented at an angle of $10^{\circ}$ from the surface normal.

\section{B.2 Pressure Cell}


The pressure cell used in the present study is shown in Fig. 1. It consisted of an optical flat and a plano-convex lens which were polished to $1 / 10$. The nanometer scale topography of these optical surfaces was examined by AFM. On the scale of the contact diameter, the surfaces were observed to have large (200 $\AA$ deep, 0.3 to $1 \mathrm{~mm}$ wide) polishing scratches crisscrossing the surface with an average separation of $5 \mathrm{~mm}$. The smaller scale roughness in the flat areas between scratches consisted of smaller scratches and bumps with an rms height of $\sim 0.5 \mathrm{~mm}$. At pressures that created an optical contact area of $0.5 \mathrm{~mm}$ radius $(\sim 50 \mathrm{MPa})$, these small asperities would have deformed to contact diameters of about $0.5 \mathrm{~mm}$, which is the length scale of their separation. Therefore, we are confident that the monolayer molecules between the two surfaces in the contact area were under nearly uniform pressure during our optical measurements, except for those adsorbed in the deep scratches, which accounted for a negligible fraction of the total amount.

Only the flat window was coated by the monolayer. The radius of curvature of the lens was chosen according to the desired pressure range, and was typically between 0.5 $\mathrm{cm}$ and $50 \mathrm{~cm}$. This sphere-on-plane geometry ensured that the concentrated stress at the contact point produced sufficient physical contact between the two surfaces and allowed the pressure to be applied to the monolayer. This geometry was also simple enough to allow the laser beams to enter and exit without complicated alignment procedures. The entire cell was mounted on an $X, Y, Z$ translation stage with a tilt adjustment to allow the SHG or SFG signal generated at the interface to enter the detection system and the sample to be scanned. The size of the contact area could be adjusted between $600-1000 \mu \mathrm{m}$ by the loading spring from the lens side. The contact area was readily observed by the 
disappearance of the reflected light since both the lens and the flat window were made of the same material. If the contact area was smaller than the laser beam spot, a doughnutshaped laser reflection was observed. If the contact area was larger than the laser beam spot, no linear reflection was observed. This disappearance of reflected light also ensured that the contact area was of good optical quality. The nonlinear optical signal was, by arrangement, only generated by the monolayer in the contact area and could radiate in both forward and reflected directions. The experiments were carried out using two methods. The first was to measure the signal generated from the monolayer at the flat optical surface first with the lens far away and subsequently with the lens in contact with the flat by compressing the spring. The size of the contact increased as the pressure was increased and eventually reached a size significantly larger than the laser beam spot. The second method was to measure the variation of the SHG or SFG signal as the laser spot was scanned over the contact area. The reversibility of the molecular characteristics under pressure was also studied by removal of the pressure.

\section{B.3 Materials}

A variety of films of different molecules were prepared for these experiments. They formed either self-assembled monolayers (n-Octadecyltriethoxysilane, OTE), or Langmuir-Blodgett films (stearic acid, octadecylalcohol, octadecylamine and 4'-n-octyl-4cyanobiphenyl (8CB)). OTE was purchased from Huls America, Piscataway, N.J. It was vacuum distilled and filtered through $0.2 \mathrm{mM}$ PTFE membranes immediately prior to preparation of prehydrolysis solutions. Stearic acid, octadecylalcohol and octadecylamine were purchased from Sigma and recrystallized from ethanol. $8 \mathrm{CB}$ was purchased from 
Merck and used as received. For our control experiments, we used a fluorescent dye molecule, octadecylrhodamine $B$ chloride salt $\left(\mathrm{C}_{18} \mathrm{Rh}\right)$, which was purchased from Molecular Probes and used as received.

The water used was house-distilled and passed through a four-cartridge Millipore $\mu \mathrm{QF}$ purification train, producing a resistivity of $18.2 \mathrm{M} \Omega \cdot \mathrm{cm}$. Tetrahydrofuran (THF) and cyclohexane were spectral quality. Glassware used for preparation of prehydrolysis solutions and for self-assembly of OTE were cleaned with nochromix reagent immediately prior to use.

\section{B.4 Film Preparation Methods}

IR quartz disks were cleaned in fresh chromic acid solution (ca. 1:5 saturated sodium dichromate: sulfuric acid), followed by extensive rinsing with Millipore water. The self-assembled OTE films were prepared by hydrolysis of the triethoxy groups to alcohol. ${ }^{23}$ Prehydrolysis solutions were prepared by dissolving $0.2 \mathrm{~g}$ OTE (19 $\mathrm{mM}$ ) in 25 $\mathrm{ml}$ THF containing $0.2 \mathrm{~g}$ of $1 \mathrm{~N} \mathrm{HCl}$. The solution was stirred at room temperature for 23 days. The prehydrolysis solution was filtered through a $0.2 \mathrm{mM}$ nylon or PTFE membrane followed by dilution with $25 \mathrm{ml}$ of cyclohexane. This solution was joined with the freshly cleaned quartz disks. Following self-assembly, the samples were briefly rinsed with fresh cyclohexane, dried under a stream of argon or nitrogen and stored in cyclohexane until used.

Langmuir-Blodgett deposition was carried out using a standard trough deposition system (KSV, Finland) filled with pure de-ionized water. All monolayers were compressed at $10 \mathrm{~mm} / \mathrm{min}$ to the desired pressure and transferred to the quartz support 
using an upstroke transfer speed of $5 \mathrm{~mm} / \mathrm{min}$. The $8 \mathrm{CB}$ monolayers (compound 1 ) were deposited at $10 \mathrm{mN} / \mathrm{m}$, with a transfer ratio of 0.93 . Stearic acid (2) monolayers were transferred at $25 \mathrm{mN} / \mathrm{m}$, with a transfer ratio of 1.0 . For fluorescence studies, stearic acid monolayers were mixed with 0.2 mole \% octadecylrhodamine (5) No changes were observed in the pressure-area isotherm after addition of the fluorescent lipid. Octadecylamine (3) monolayers were transferred at $15 \mathrm{mN} / \mathrm{m}$, with a transfer ratio of 1.0 . Octadecylalcohol (4) monolayers were transferred at $25 \mathrm{mN} / \mathrm{m}$, with a transfer ratio of 1.0.

\section{Results \\ C. 1 Control Experiments}

Control experiments were carried out to ascertain that reductions in the SHG and SFG signals arise from genuine changes in the monolayer and are not due to artifacts such as optical distortions caused by the applied pressure, or to the molecules being displaced out of the contact area. Optical distortions could be caused, for example, by inhomogeneous changes in the refractive index of the flat and lens across the contact area due to the elastic deformation of asperities and protrusions. This could distort the wavefront of the incident light (loss of spatial coherence) and cause the surface oscillators to radiate randomly out of phase, leading to a decrease of the detected signal.

To determine if the non-linear optical signal is affected by optical effects resulting from the formation of the contact, we performed an experiment with crystalline quartz pressed between the lens and the flat. In this experiment, the laser beam was incident from the lens side to avoid bulk contribution to the SHG from the crystalline quartz. The 
second-order non-linear susceptibility of the crystalline quartz should not change with pressure. In the reflection geometry, the SHG signal came only from the very thin layer near the surface due to the short coherence length for this particular geometry. Therefore, it resembled the geometry of the trapped monolayers in the contact area since, in both cases, the SFG arose from the contact surface region. Since crystalline quartz has a nonvanishing component $\chi_{\mathrm{xxx}}^{(2)}$, we used an s-in, s-out polarization geometry and aligned s parallel to the crystal $\mathrm{x}$-axis. The SHG signal arising from the contact area was found to be $\sim 3$ times larger than before contact was made. A theoretical calculation using the formulas given in the introduction indicates that changes in the local field factor alone can explain our observation. A factor of 5 is expected from $S \propto\left[L_{y y}(2 \omega) \chi_{x x}^{(2)} L_{y y}^{2}(\omega)\right]^{2}$ for a gap width $d=0$ compared to $d=\infty$. The slight disagreement between the experimental data and the calculation may be due to the alignment of the outgoing beam, which was made difficult by the curvature of the lens. This result indicates that pressure-induced phase variations across the contact are negligible and that the theory used to treat such a contact geometry described in the introduction is valid.

The second control experiment was aimed at verifying that the monolayer molecules remain trapped in the contact area during compression and are not displaced outside. For this purpose, we performed a fluorescence microscopy experiment with a Langmuir-Blodgett film of stearic acid mixed with $0.2 \%$ octadecylrhodamine. Since the Rhodamine was attached to the end of an alkyl chain similar to that of the host (stearic acid), the binding of both molecules to the substrate and to the rest of the film was similar. The apparatus, described in Sec. B.1, provided illumination at a wavelength of $480 \mathrm{~nm}$. 
This is suitable for excitation of Rhodamine which has an excitation spectrum peak at 540 $\mathrm{nm}$, but has a broad tail that extends to shorter wavelength. The emitted light was filtered to pass the fluorescence band of Rhodamine at $600 \mathrm{~nm}$ and detected by a photomultiplier tube. Background fluorescence was measured in the absence of the monolayer, and later subtracted from the monolayer signal. Using a beam size of $300 \mu \mathrm{m}$, and a contact area of $500 \mu \mathrm{m}$, no florescence signal loss was detected by scanning from outside to inside the contact area during compression. This clearly indicates that no molecules were excluded by pressure from the contact area.

\section{C.2 8CB Monolayers Under Pressure}

We used second harmonic generation to study the behavior of a LangmuirBlodgett monolayer of the liquid crystal $8 \mathrm{CB}$ under pressure. This rod-like molecule consists of a cyanobiphenyl head and an alkyl chain of 8 carbon atoms. Previous studies ${ }^{20}$ have shown that the molecular polarizability responsible for SHG comes mainly from the polar head of the molecule (i.e., the cyanobiphenyl group) and has only one dominant element. Furthermore, the monolayer has azimuthal symmetry.

Fig. 2 shows the intensity of the SHG signal generated at various positions inside and outside the contact area probed by the laser beam with a $100 \mu \mathrm{m}$ spot size. The average pressure in the contact was about $20 \mathrm{MPa}(\mathrm{a} \sim 300 \mathrm{~mm}, \mathrm{R} \sim 20 \mathrm{~cm})$. The oscillation outside the contact was caused by the interference effect on the fundamental input and can be well accounted for by the local field factors as a function of the gap separation between the surfaces of the lens and the flat, as shown by the solid line. For the m(mixed polarization, $45^{\circ}$ between $s$ and $p$ )-in/p-out polarization combination, the nearly vanishing 
signal (taking into account the limited signal-to-noise ratio) in the contact area can be well explained by the vanishing local field factors (Fig. 2a). For the m-in/s-out polarization combination, the local field factors predict only a factor of $\sim 2.5$ decrease in signal inside the contact spot. As shown in Fig. 2b, a much larger decrease in signal (at least a factor of 100) was observed. With a larger laser beam spot filling almost the entire contact area, a better signal-to-noise ratio was obtained, which allowed us to say that the decrease of the signal was at least a factor 1000 . Such a significant change must result from the vanishing of the nonlinear susceptibility.

Retraction of the lower lens after these measurements to reduce the pressure caused the SHG signal to reappear with only a slight decrease in intensity.

\section{C.3 Sum Frequency Generation Experiments}

The spectral region of the $\mathrm{CH}$ stretching modes of LB monolayers of stearic acid, octadecylalcohol, octadecylamine and of a self-assembled monolayer (SAM) of OTE before contact were found to be identical. Fig. 3 shows the SFG spectrum in the $\mathrm{CH}$ stretch region for a LB monolayer of octadecylalcohol on the fused quartz surface before contact. It arises solely from the polar-ordered end group $\mathrm{CH}_{3}$, and contribution from the $\mathrm{CH}_{2}$ groups cancels out because of pairing in opposite directions. This suggests that all these monolayers were closely packed so that the hydrocarbon chains were in an all-trans configuration. The peaks at 2875 and $2940 \mathrm{~cm}^{-1}$ have been assigned to the symmetric $\mathrm{CH}_{3}$ stretching mode and a Fermi resonance between stretch and overtone of bend vibrations of $\mathrm{CH}_{3}$, respectively. ${ }^{7}$ For a octadecylalcohol monolayer, when the normal force was slowly increased, it was found that the SFG signal decreased continuously as the 
radius of the contact area was increased. When the contact area equaled that of the laser beam size, the signal fell below our detection limit, set by the scattered background light of the visible laser. The change of SFG signal was observed to be almost completely reversible as the pressure was varied and could be repeated for many cycles of increasing and decreasing pressure. At the end of $\sim 10$ cycles, a small permanent reduction in the signal occurred, indicating perhaps material loss due to unwanted shearing during pressure loading and unloading despite the attempts in designing the cell to avoid such lateral motions. In Fig. 4a, we show the SFG signal of the $\mathrm{CH}_{3}$ peak at $2875 \mathrm{~cm}^{-1}$ as a function of sample position across the contact area for a film of octadecylalcohol with a contact size of $\sim 1 \mathrm{~mm}$. The average pressure in the contact area was about $50 \mathrm{MPa}$. Similar to the $8 \mathrm{CB}$ case in the SHG study, the SFG signal in the contact area drops by about a factor of 1000 to below the noise level. Outside the contact area, the SFG signal oscillates due to interference as discussed in the previous section. The SFG signal from $\mathrm{OH}$ at $3250 \mathrm{~cm}^{-}$ ${ }^{1}$ for the same monolayer was also found to drop below the noise level inside the contact area as shown by Fig. $4 \mathrm{~b}$. It should be noted that there may be a contribution to the $\mathrm{OH}$ stretch signal from $\mathrm{H}_{2} \mathrm{O}$ molecules that may have been transferred with the $\mathrm{LB}$ film. If this were the case, our experiment suggests that these water molecules must have been displaced out of the contact area or randomized by pressure. Although qualitatively similar observations were obtained for the $\mathrm{CH}_{3}$ stretch peak of SAM OTE films, a significant difference is that a finite SFG signal inside the contact area remained under the same load, as is shown in Fig. 5. At higher loads $(\sim 80 \mathrm{MPa})$, the $\mathrm{CH}_{3}$ signal disappeared as in the previous cases. 
The consistent result from all these experiments is that the monolayer was unable to generate an SFG signal under high enough pressure. These results imply that, as in the case of SHG, the loss of SFG signal arises from the vanishing of the non-linear susceptibility of the film.

\section{Discussion}

\section{D.1 Confinement and Compression of Molecules}

The fluorescence experiment described above proves very clearly that the molecules confined between the lens and the flat are not displaced outside of the contact area. In this experiment, Langmuir-Blodgett films were used that are bound to the substrate by only weak van der Waals forces. In other experiments where self-assembled monolayers were used, the binding to the substrate was covalent and displacement of the molecules was even more unlikely, as it would require higher energy.

The response of organic monolayers to local pressure, as exerted by the sharp tip of an AFM, has been studied recently in our laboratory. Using alkylthiols on $\mathrm{Au}(111)$, we have shown that sharp asperities (tip radii $<1000 \AA$ ) could reversibly displace the thiols, i.e., the thiols would refill the contact area upon release of the tip pressure. ${ }^{25}$ The critical load for the displacement was found to be around $300 \mathrm{nN}$ for tips of $700-1000 \AA$ radii, and $10 \mathrm{nN}$ for radii of $\sim 200 \AA$. This corresponds to pressures of the order of $1 \mathrm{GPa}$, which is higher than that applied in the present experiments. The displacement or nondisplacement is the result of competition between lateral compression (molecule-molecule repulsion) and molecular deformation induced by the tip pressure. ${ }^{25}$ If tip radii larger than $1000 \AA$ were used, no displacement of the thiols occurred. In fact, the gold substrate was 
found to yield plastically before the organic layers could be displaced. ${ }^{26}$ Similar studies performed on self-assembled monolayers of OTE on mica showed that displacement of the covalently cross-linked alkyl chains was much more difficult. For example, while the threshold load for thiol displacement was about $5 \mathrm{nN}$ for $\sim 100 \AA$ tips, a load of $10 \mathrm{nN}$ was required for the OTE films using the same tips. ${ }^{27}$

\section{D.2 Vanishing of Second-Order Monolayer Susceptibility}

The decrease of the intensity of the SFG and SHG signals was found to be much more significant than predicted by changes in the local field (Eq. 6.5). We can calculate the changes due to the local field factors to predict how they would affect the SFG and SHG signals outside and inside the contact area. This is shown in Fig. 2 for SHG from $8 C B$. The solid curves are the result of calculations using Eq. (6.5), assuming that no change in the $8 \mathrm{CB}$ monolayer susceptibility has occurred. Inhomogeneities in the film cause a signal imbalance between the left and right side of the contact. The oscillation of the signal outside the contact region fits well with the calculation. It arises from the dependence of $\mathrm{L}_{\mathrm{ii}}(\omega)$ on the distance between the surfaces of the lens and the flat, averaged over the laser beam size of $100 \mu \mathrm{m}$. A sizable signal is predicted from the calculation for the m-in/s-out polarization combination inside the contact area. The fact that the experimental value for this polarization combination drops to zero inside the contact area indicates that the second order susceptibility $\chi_{s}^{(2)}$ of the monolayer must have been reduced to zero by the pressure used in the experiment. This leads us to conclude that the biphenyl core groups of the $8 \mathrm{CB}$ molecules must lie flat on the substrate as a result of the pressure $\left(\theta=90^{\circ}\right)$ in Eq. (6.9). This is possible because the $8 \mathrm{CB}$ monolayer 
is not too densely packed ( $\sim 35 \AA$ per molecule), which allows for such a reorientation of the molecules.

In the case of SFG from $\mathrm{CH}_{3}$, the second-order nonlinear susceptibility is given by Eq. (6.9). If we assume that the axis of $\mathrm{CH}_{3}$ group is reoriented under the applied pressure, such that both $<\cos \theta>$ and $<\cos ^{3} \theta>$ vanish, then the disappearance of the $\mathrm{CH}_{3}$ spectrum in SFG from molecules with long alkyl chain under pressure can be explained. A similar reasoning can be used to understand the disappearance of the $\mathrm{OH}$ stretch mode of the octadecylalcohol monolayer under pressure. Since these changes occur at the ends of the long hydrocarbon chain, they could recover relatively easily. This elastic recovery of molecular films subjected to moderate pressure has been observed with the surface force apparatus $^{28}$ and also in many AFM experiments with tip pressures of up to $1 \mathrm{GPa}^{8-9,24-26}$ Since our pressures were below this value, our assumption that molecular distortions at the head and/or tail groups recover upon release of the applied pressure appears well justified.

A recent Monte Carlo simulation ${ }^{29}$ of self-assembled thiol monolayers suggests that the molecular deformation is in the form of gauche defects, in addition to an increase of the molecular tilt. This simulation also concludes that the gauche defects are essentially concentrated at the molecular ends. With increasing pressure during compression, a dramatic increase (from $6 \%$ to $38 \%$ ) in the number of molecules with such end gauche defects is observed.

These results agree with our observations and the picture presented. We note that a chain oriented close to the surface normal with one single gauche defect at the terminal 
C-C bond will have the end group oriented with its $\theta$ close to $90^{\circ}$ and therefore will not contribute to the surface nonlinear susceptibility.

If the hydrocarbon chains contain considerable conformational defects, peaks corresponding to $\mathrm{CH}_{2}$ stretches might be observable due to the break down of inversion symmetry. Such is the case for an uncompressed monolayer of fatty acid on a water surface. ${ }^{7}$ However, in our experiments, the $\mathrm{CH}_{2}$ stretch was not observed even when the pressure was $\sim 0.8 \mathrm{GPa}$. One explanation is suggested by the Monte Carlo simulation study, ${ }^{28}$ where the actual density of internal conformational (gauche) defects is very low. Another possibility is that the system, with the molecular monolayer sandwiched between two quartz surfaces, does not break the inversion symmetry seen by the $\mathrm{CH}_{2}$ groups, unlike the case for molecules at the air/water interface. ${ }^{7}$

In conclusion, we have shown for the first time that SHG and SFG can be used to provide orientational information on monolayers of molecules under high static pressure. We have shown that for Langmuir-Blodgett and self-assembled monolayers, relatively low pressure is needed to alter the end configuration of the chains, and that this change is completely reversible. For monolayers such as $8 \mathrm{CB}$ that are less densely packed, it is possible that the molecules are pressed flat on the surface. 


\section{References}

1. (a) A. Kono, Lubrication 32, 255 (1987); (b) V. Depalma, N. Tilmann, Langmuir 5, 868 (1989); and references therein.

2. E. Rabinowicz, Tribology and Mechanics of Magnetic Storage Systems; B. Bhushan, N.S. Eiss, STLE, Vol. 3, p. 1 (1986).

3. A.M. Homola, C.M. Mate, and G.B. Street, MRS Bull. 45 (1990).

4. G.G. Roberts, Adv. in Phys. 34, 475 (1985); G.M. Whitesides and P.E. Laibinis, Langmuir 6, 87 (1990); and references therein.

5. J.F. Rabolt, F.C. Burns, N.E. Schlotter and J.D. Swalen, J. Chem. Phys. 78, 946 (1983); D.L. Allara and R.G. Nuzzo, Langmuir 1, 52 (1985); and references therein.

6. J.A. Zasadzinski, R. Viswanathan, L. Madsen, J. Garnaes, and D.K. Schartz, Science 263, 1226 (1994), and references therein.

7. P. Guyot-Sionnest, J.H. Hunt and Y. R. Shen, Phys. Rev. Lett. 59, 1597 (1988).

8. S.A. Joyce, R.C. Thomas, J.E. Houston, T.A. Michalske and R.M. Crooks, Phys. Rev. Lett. 68, 2790 (1992).

9. N.A. Burnham, D.D. Dominguez, R.L. Mowery and R.J. Colton, Phys. Rew. Lett. 64, 1931 (1990).

10. G.S. Blackman, C.M. Mate and M.R. Philpott, Phys. Rev. Lett. 65, 2270 (1990).

11. J.N. Israelachvili, Surf. Sci. Rep. 14, 109 (1992).

12. See, for an example, J.L. Lauer and B.L. Vleck, in Surfaces Science Investigations in Tribology, ACS Symp. Ser. 485 , p. 112 (1992). 
13. J. Ruhe, V.J. Novotny, K.K. Kanazawa, T. Clarke, and G.B. Street, Langmuit 9, $2383(1993)$.

14. Q. Du, E. Freysz, and Y.R. Shen, Science 264, 826 (1994).

15. S. Ong, X. Zhao, and K.B. Eisenthal, Chem. Phys. Lett. 191, 327 (1992).

16. K.L. Johnson, Contact Mechanics, Cambridge University Press (1985).

17. Handbook of Chemistry and Physics, 49th edition, F57 (1968).

18. Y.R. Shen, Ann. Rev. Phys. Chem. 40, 327 (1989); Y.R. Shen, Nature (London) 337, $519(1989)$

19. X.D. Zhu, H. Suhr, and Y.R. Shen, Phys. Rev. B 35, 3047 (1987); J.H. Hunt, P. Guyot-Sionnest and Y.R. Shen, Chem. Phys. Lett. 133, 189 (1987).

20. Y.R. Shen, Liquid Crystals 5, 635 (1989); C.S. Mullin, P. Guyot-Sionnest and Y.R. Shen, Phys. Rev. A 39, 3745 (1989); and references therein.

21. R. Superfine, J.Y. Huang and Y.R. Shen, Phys. Rev. Lett. 66, 1066 (1991).

22. M. B. Feller, W. Chen, and Y.R. Shen, Phys. Rev. A 43, 6778 (1991).

23. C.R. Kessel and S. Granick, Langmuir 7, 532 (1991).

24. G.-y. Liu and M. Salmeron, Langmuir 10 (2), 367 (1994).

25. M. Salmeron, G.-y. Liu and D.F. Ogletree, in Forces in Scanning Probe Methods, eds. H.-J. Günterodt, D. Anselmetti and E. Meyer. NATO ASI Series E: Applied Sciences. Kluwer Academic Publishers, The Netherlands. In press (1994).

26. M. Salmeron, G. Neubauer, A. Folch, M. Tomitori, D.F. Ogletree and P. Sautet, Langmuir 9 (12), 3600 (1993).

27. X.D. Xiao, G.-y. Liu, D. H. Charych, and M. Salmeron, Langmuir, in press. 
28. Y.L. Chen, C.A. Helm and J.N. Israelachvili, Langmuir 7, 2694 (1991).

29. J.I. Siepmann and I.R. Mcdonald, Phys. Rev. Lett. 70, 453 (1993). 


\section{Figure Captions}

Fig. 1. Schematic diagram of the apparatus used to measure the non-linear optical properties of molecular films under uniaxial pressure. An optically flat, fused quartz window with a monomolecular film is placed at the top of the cell. Contact between the flat and a lens is established by pushing the movable lens with a spring that is compressed using a micrometer. Elastic deformation creates a contact area with a diameter of roughly 0.1 to $1 \mathrm{~mm}$ with pressures of the order of $50 \mathrm{MPa}$. The laser beams are incident from the flat quartz window and focused onto the contact interface.

Fig. 2. Second Harmonic Generation (SHG) signal from a $8 \mathrm{CB}$ film subjected to an average compressive pressure of $20 \mathrm{MPa}$, as a function of the beam position across the contact area (radius $\sim 300 \mu \mathrm{m}$ ). (a) $\mathrm{M}$ (mixed) polarization is used for the incident beam ( $\omega)$ and S polarization for the outgoing beam (2 $\omega)$. The signal is near the noise level inside the contact and oscillates outside. (b) Same for $\mathrm{M}$ polarization in and $\mathrm{P}$ polarization out. In both cases, the predicted signal due to changes from the local field (see text) alone are shown by the continuous line.

Fig. 3. Sum Frequency Generation (SFG) signal versus $\mathrm{IR}$ frequency in the $\mathrm{C}-\mathrm{H}$ stretch region for a Langmuir-Blodgett film of octadecylalcohol on a flat quartz surface. The peak at around $2875 \mathrm{~cm}^{-1}$ is due to the symmetric $\mathrm{C}-\mathrm{H}$ 
stretch of the terminal $\mathrm{CH}_{3}$ group. The peak at approximately $2940 \mathrm{~cm}^{-1}$ is a Fermi resonance between $\mathrm{CH}_{3}$ symmetric stretching and the overtone of its bending modes.

Fig. 4 (a) SFG signal of the $\mathrm{CH}_{3}$ symmetric stretch mode of an octadecylalcohol Langmuir-Blodgett monolayer on a flat fused quartz versus laser spot position in the contact area. (b) Same experiment for the $\mathrm{OH}$ stretch mode. Inside the contact, the SFG decreases by a factor of 1000 to within the noise level. Oscillations in the outer region are due to interferences. Average pressure $\sim 50$ $\mathrm{MPa}$.

Fig. 5. SFG signal of the $\mathrm{CH}_{3}$ symmetric stretch mode of a self-assembled monolayer of OTE adsorbed on the flat fused quartz window versus laser beam position across the contact area. At $50 \mathrm{MPa}$ pressure, the intensity inside the contact area decreases by a factor of approximately 15. At the higher pressure of $80 \mathrm{MPa}$, the decrease is at least a factor 1000 , to within the noise level. 


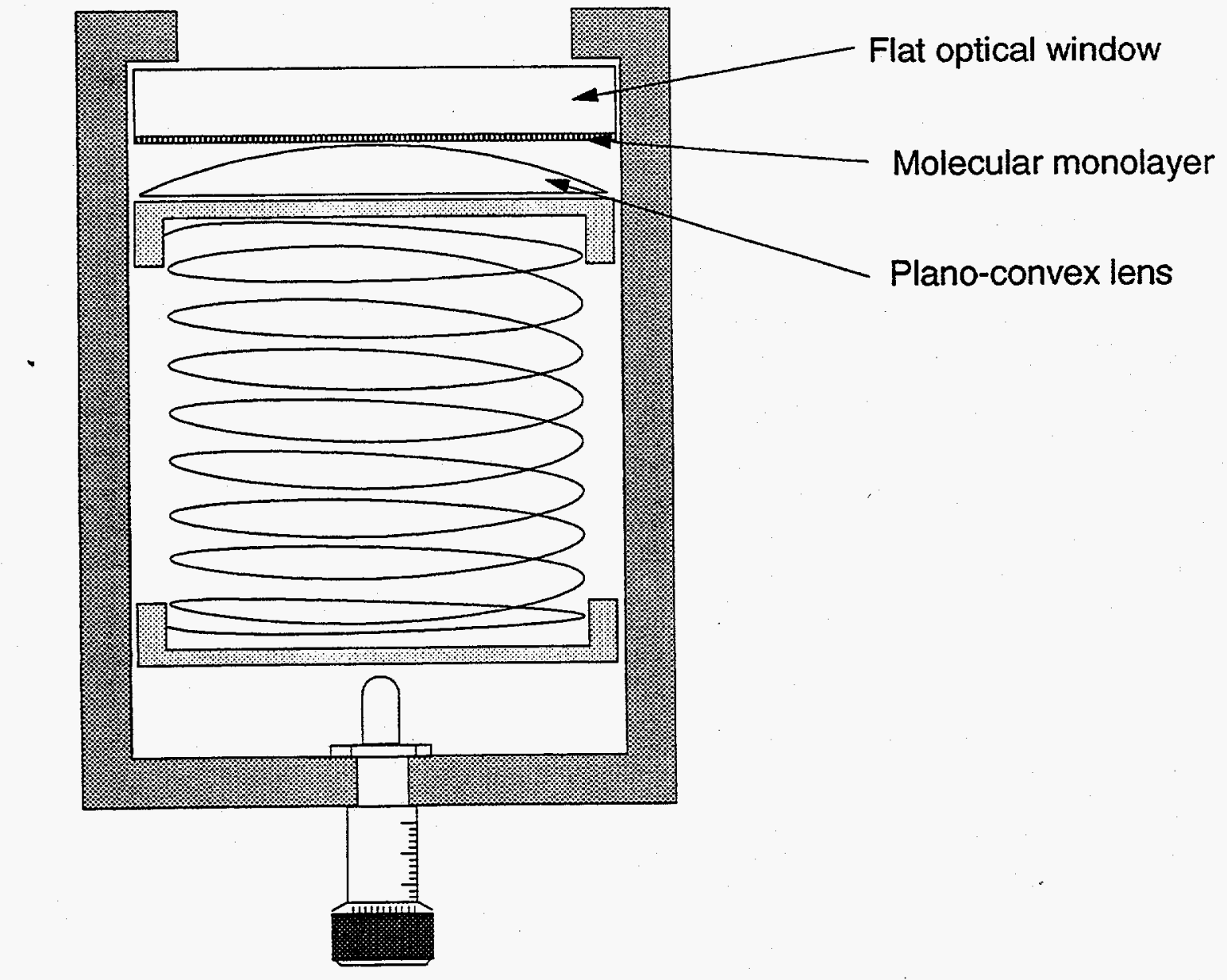

Fig. 1 


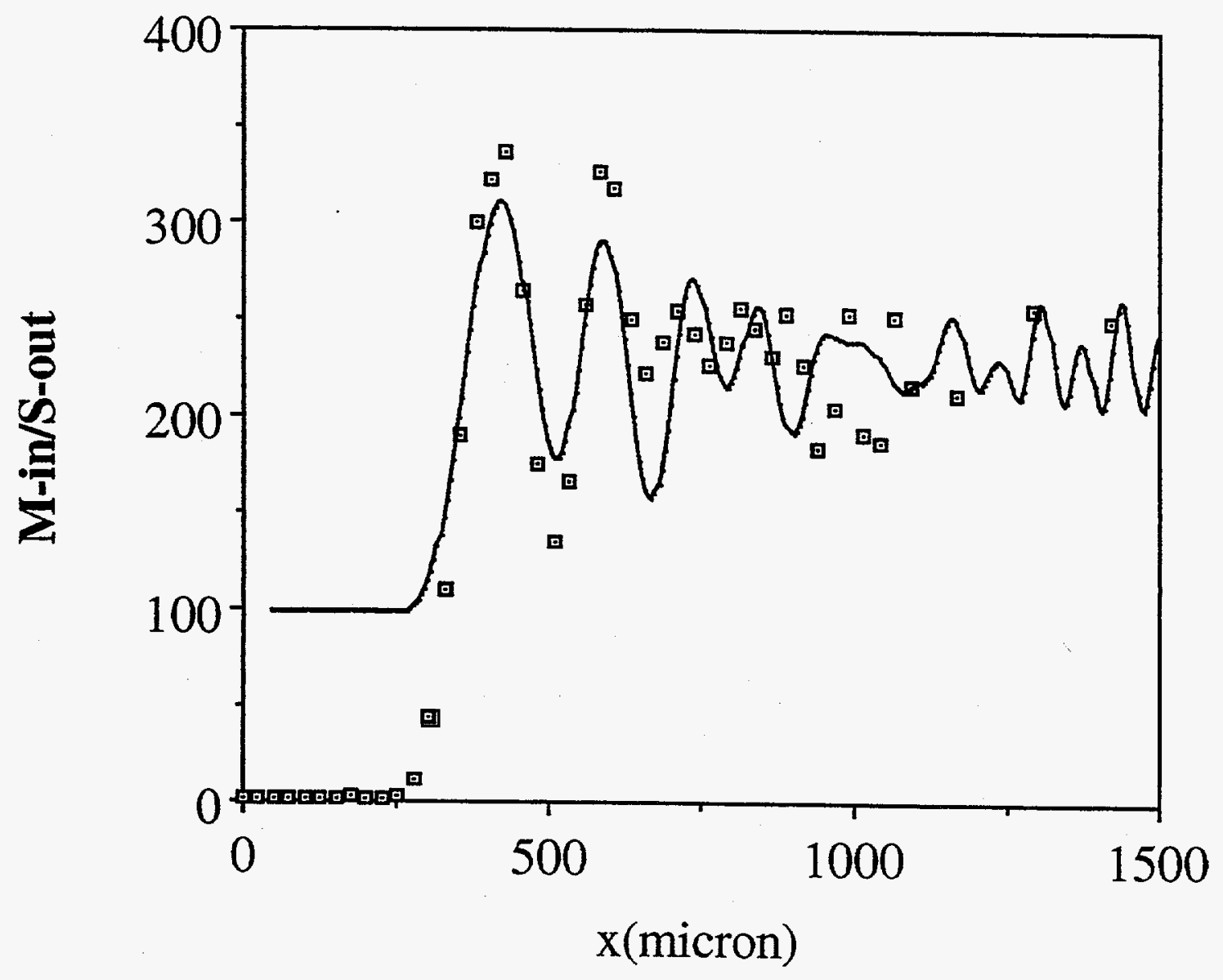

Fig. $2 a$ 


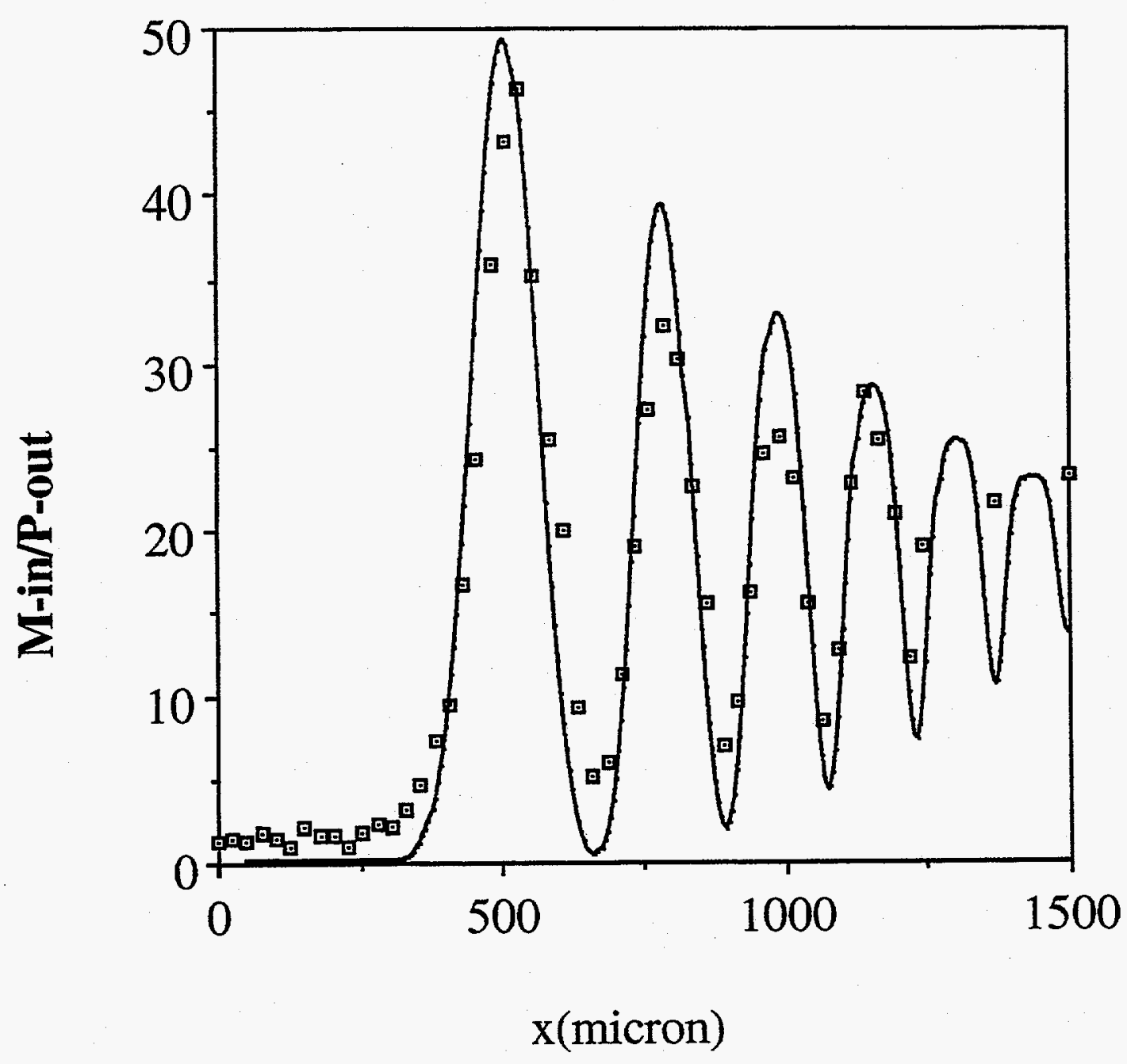

Fig. 2b 


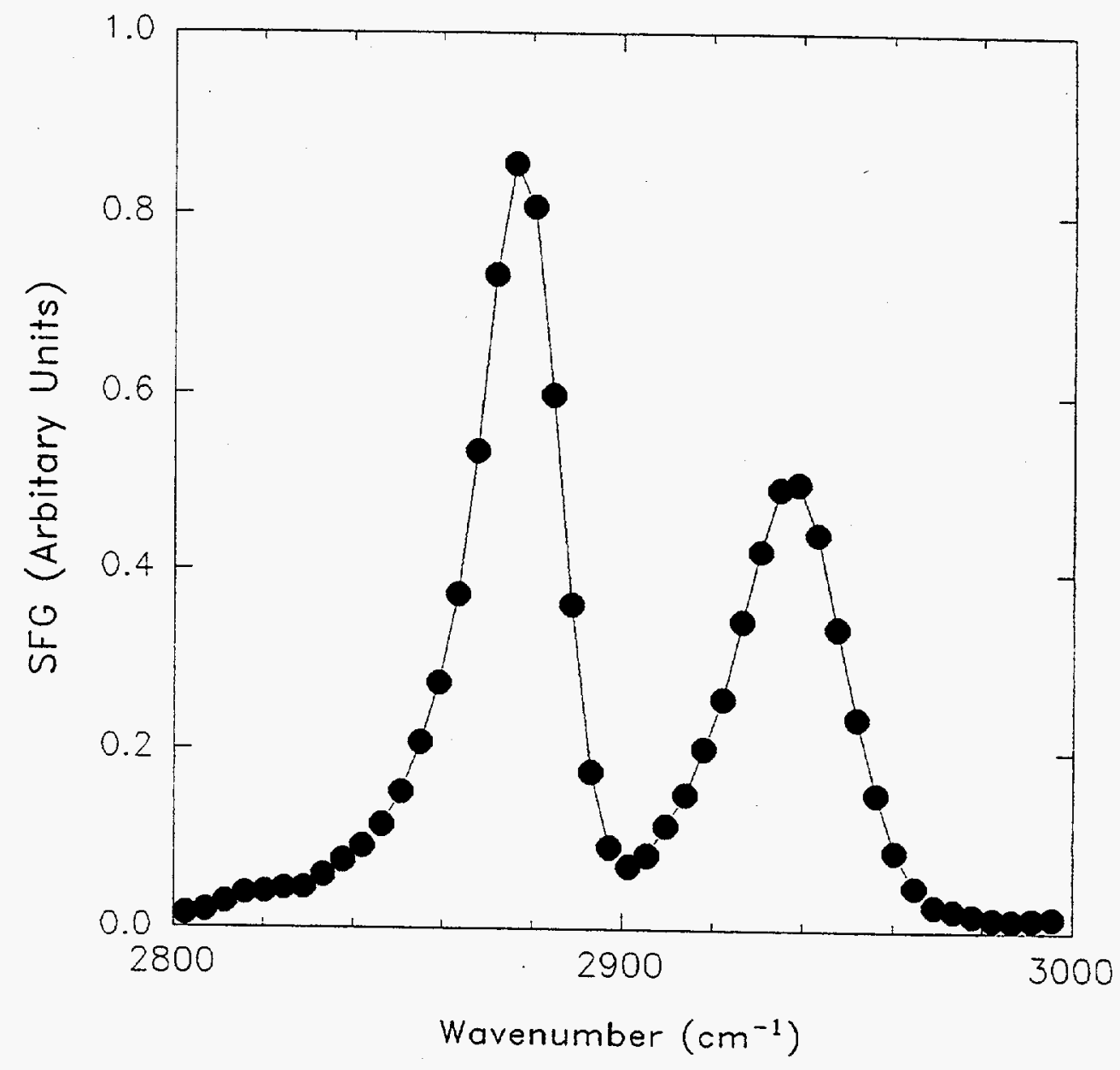

Fig. 3 


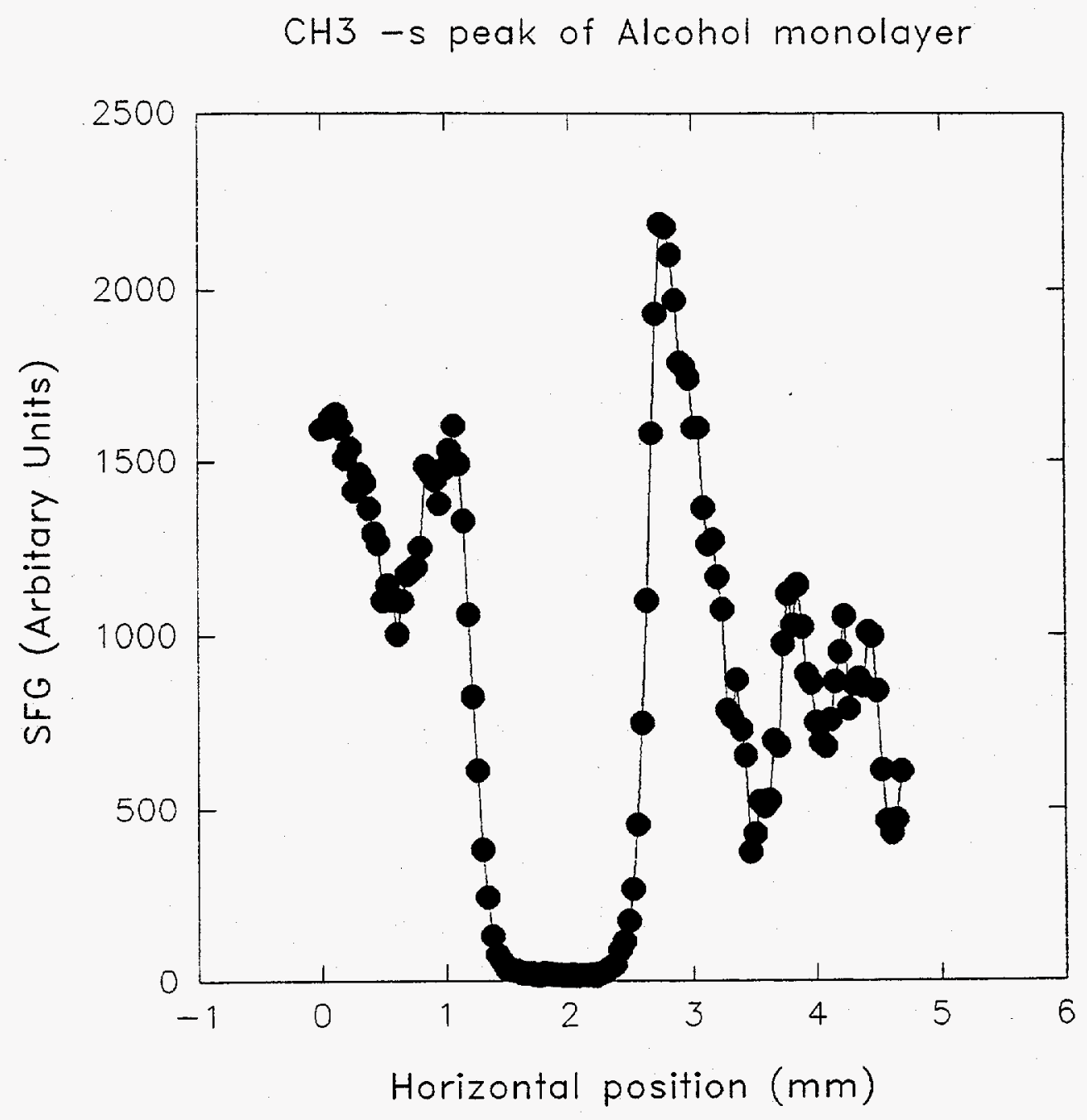

Fig. 4a 


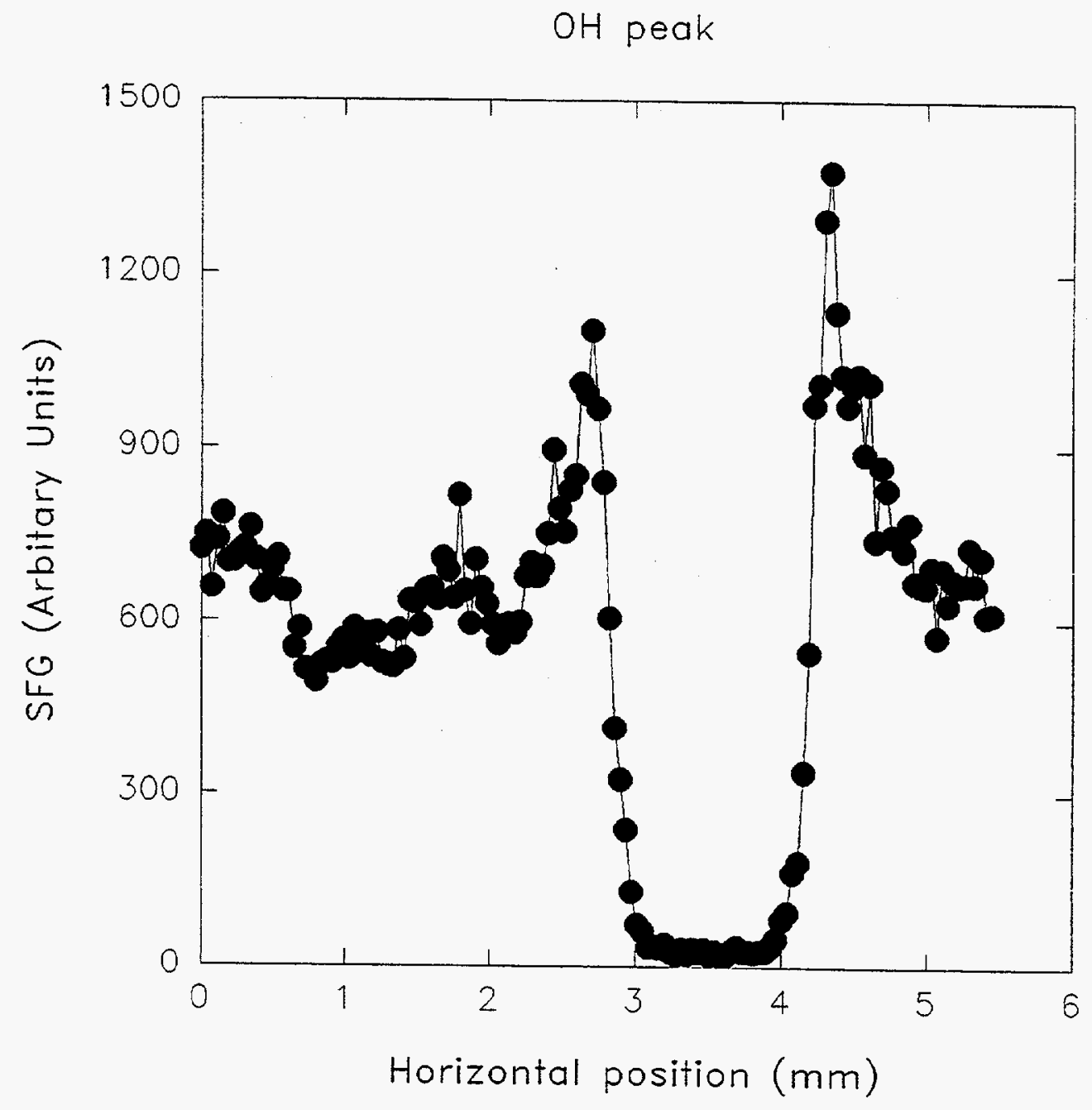

Fig. $4 b$ 
$\mathrm{CH} 3$-s peak of OTS monolayer

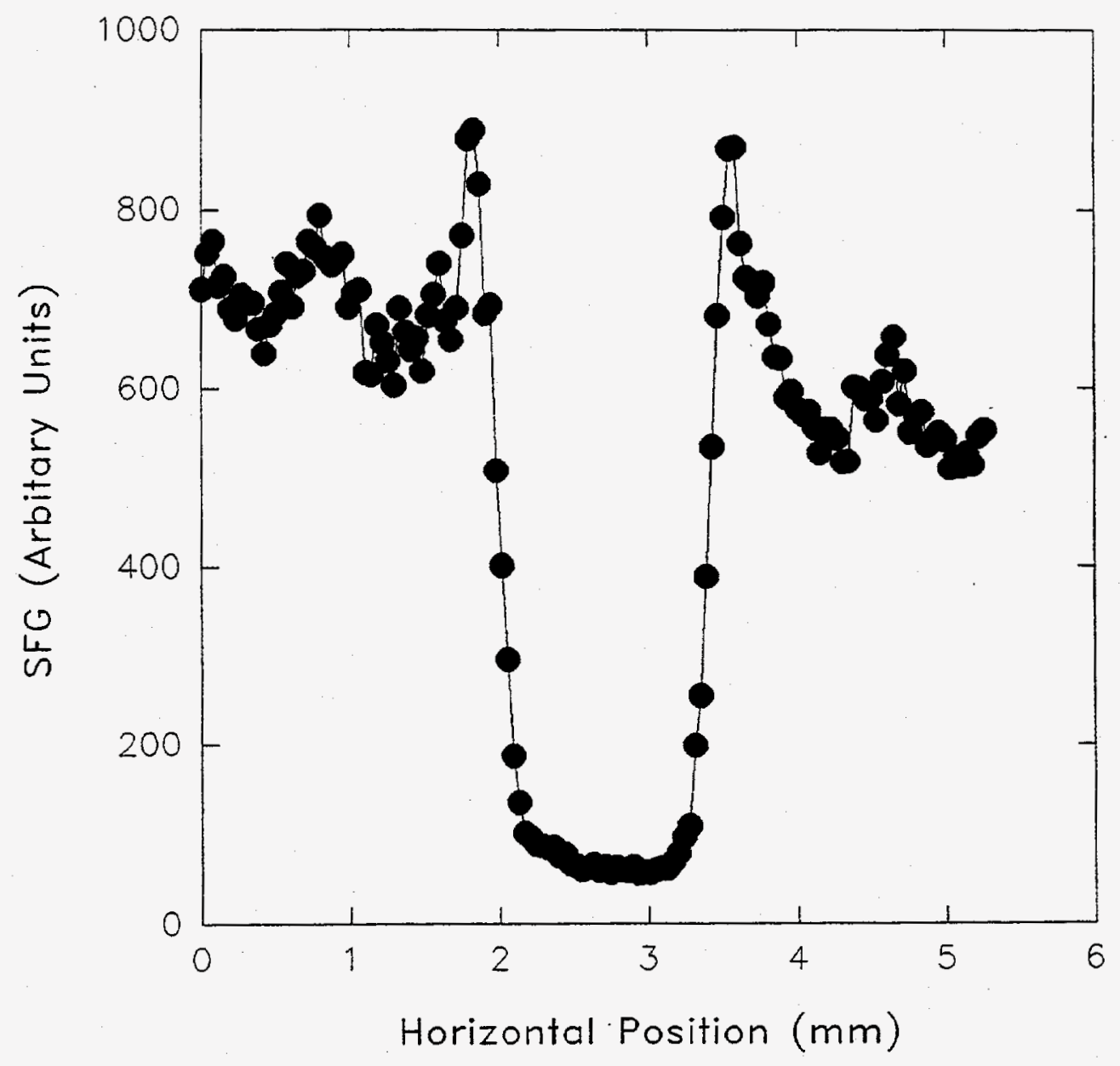

Fig. 5 